\title{
17. CENOZOIC RADIOLARIA FROM THE CARIBBEAN, DEEP SEA DRILLING PROJECT, LEG 15
}

\author{
William R. Riedel and Annika Sanfilippo, Scripps Institution of Oceanography, La Jolla, California
}

\section{INTRODUCTION}

Of the sites occupied during Leg 15 of the Deep Sea Drilling Project, the following ones yielded significant numbers of radiolarians:

Site $146-15^{\circ} 06.99^{\prime} \mathrm{N}, 69^{\circ} 22.67^{\prime} \mathrm{W}$; water depth 3949 meters

Site $149-15^{\circ} 06.25^{\prime} \mathrm{N}, 69^{\circ} 21.85^{\prime} \mathrm{W}$; water depth 3972 meters

Site $150-14^{\circ} 30.69^{\prime} \mathrm{N}, 69^{\circ} 21.35^{\prime} \mathrm{W}$; water depth 4545 meters

Site $151-15^{\circ} 01.02^{\prime} \mathrm{N}, 73^{\circ} 24.58^{\prime} \mathrm{W}$; water depth 2029 meters

Site $152-15^{\circ} 52.72^{\prime} \mathrm{N}, 74^{\circ} 36.47^{\prime} \mathrm{W}$; water depth 3899 meters

Site $153-13^{\circ} 58.33^{\prime} \mathrm{N}, 72^{\circ} 26.08^{\prime} \mathrm{W}$; water depth 3932 meters

In preparing this report on the Leg 15 radiolarians, we have concentrated principally on the Cenozoic occurrences and have not had sufficient time to treat the Cretaceous assemblages. For the Cenozoic occurrences we present tabulations of species abundances in all of the assemblages examined, a list of radiolarian events (earliest and latest occurrences of taxa) arranged stratigraphically, and a summary range chart. The form of presentation of these results follows closely that used in the radiolarian reports for DSDP Legs 7 and 10 (Riedel and Sanfilippo, 1971; Sanfilippo and Riedel, 1973) with the exception that the present report contains no systematic section, its place being taken by a species list and footnotes to the tables.

\section{OCCURRENCES OF RADIOLARIANS}

The following paragraphs briefly describe the radiolarian occurrences, and Figure 1 summarizes this information in relation to the occurrences of calcareous microfossils. These results support the conclusion reached on the basis of information from DSDP Legs 1, 4, and 10 (Sanfilippo and Riedel, 1973, Figure 1-3) that Caribbean sediments younger than Early or Middle Miocene generally lack siliceous microfossils, whereas older strata commonly contain them.

\section{Site 146}

Radiolarians are not present in Core 1 (at 96-105 m below the sea floor). In Core 2 (at $254-263 \mathrm{~m}$ ) they are abundant and moderately well preserved-those from the upper half of the core belong in the Lychnocanoma elongata Zone and those from the lower part in the Dorcadospyris ateuchus Zone, but because the core is disturbed the transition between these zones cannot be investigated in detail.

In Cores 3 to 10 (from 406 to approximately $470 \mathrm{~m}$ ), radiolarians vary from rare to common, and they are moderately well to poorly preserved (partially dissolved, some silicified, and some perhaps zeolitized). Core 4 evidently belongs in the Buryella clinata Zone, Core 7 in the Bekoma bidarfensis Zone, and Core 10 in that part of the Paleocene left unzoned in the Leg 10 Initial Reportthe intervening cores containing radiolarians inadequate for correlation.

In Core 11 (476 to approximately $480 \mathrm{~m}$ ) the radiolarians have what is commonly regarded as a "Cretaceous aspect", but they might equally well be early Paleocene since no well-dated assemblages of that age are known. A few specimens are illustrated (Plate 4, Figure 7-12).

Practically all samples from Cores 12 to 41 (from 485 to approximately $740 \mathrm{~m}$ ) contain rare, moderately well preserved (partially dissolved) radiolarians representing a small number of taxa. Many samples (particularly those from sandy layers) from cores below Core 14 contain, in addition, common to abundant poorly preserved (usually silicified) radiolarians representing large numbers of taxa. These results, together with the fact that an orderly sequence of foraminiferal faunas is found in the cores, suggest that the sparse, better preserved radiolarians are autochthonous, and that the floods of poorly preserved specimens were introduced by some mechanism such as turbidity currents, which carried material not significantly older than that being deposited at this site. This explanation requires a pattern of paleoecological conditions such that the water column at this site was inhabited by a restricted radiolarian assemblage, while at the presumably not-far-distant source of the transported component a much more diverse fauna flourished.

\section{Site 149}

Very rare, well preserved radiolarians are present in Core 2 (at $1-10 \mathrm{~m}$ below the sediment surface), and below that they are absent through Core 21 (at 176-185 m). Core 22 (at $185-195 \mathrm{~m}$ ) has very rare, poorly preserved fragments (badly dissolved) which are insufficient for a zonal assignment. In Cores 23 through 30 (at 195 to approximately $263 \mathrm{~m}$ ), radiolarians are few to common and moderately preserved (somewhat dissolved). Cores 31 through 42 (at 270 to approximately $376 \mathrm{~m}$ ) contain abundant, well preserved radiolarians, and in Core 43 (at 381 to approximately $382 \mathrm{~m}$ ) they are few to common, somewhat dissolved.

Core 23 (at $195-203 \mathrm{~m}$ ) is in the Lychnocanoma elongata Zone, and below that the zonal boundaries are as follows:

Base of the Lychnocanoma elongata Zone in the lower part of Core 26 (at approximately $227 \mathrm{~m}$ ).

Base of the Dorcadospyris ateuchus Zone in the lower part of Core 28 or the upper part of Core 29 (at approximately $250 \mathrm{~m}$ ). 


\begin{tabular}{|c|c|c|c|c|c|c|c|c|c|}
\hline \multirow[b]{2}{*}{ Age } & \multirow[b]{2}{*}{ Foram Zones and Subzones } & \multicolumn{8}{|c|}{ Sites } \\
\hline & & $\begin{array}{l}149, \\
146\end{array}$ & 147 & 148 & $\begin{array}{l}150, \\
150 \mathrm{~A}\end{array}$ & 151 & 152 & 153 & $\begin{array}{l}154, \\
154 \mathrm{~A}\end{array}$ \\
\hline Holocene & G. fimbriata & $\square_{1}$ & $\Pi^{2}$ & & & & & & \\
\hline \multirow{4}{*}{ Pleistocene } & G. bermudezi & & 3-5 & & & & & & \\
\hline & G. calida calida & \multirow{3}{*}{$\begin{array}{l}\square 2 \\
\square 3-5 \\
\end{array}$} & $\square 6-8$ & $\square 1-3$ & & & & & $\square \mathrm{Al}$ \\
\hline & G. hessi & & $\square 9-18$ & $\square 4-11$ & & \multirow{2}{*}{$\square 1$} & & & A2-7 \\
\hline & G. crassaformis viola & & & $\square 12,13$ & & & & & $\square$ A8 \\
\hline \multirow{5}{*}{ Pliocene } & G. truncat. cf. tosaensis & \multirow{6}{*}{$\prod_{6-11}$} & & $\square 14,15$ & & & & & $\square \mathrm{A9}$ \\
\hline & \begin{tabular}{|l|} 
G. exilis \\
\end{tabular} & & & 216,17 & \multirow{4}{*}{1} & & & & $\square \mathrm{A} 10$ \\
\hline & G. trilobus fistulosus & & & 员 19,20 & & & & $\square \quad 1$ & DA11 \\
\hline & G. margaritae evoluta & & & -21 & & \multirow{2}{*}{$\square^{2}$} & & & $\Pi^{\mathrm{A} 13}$ - \\
\hline & G. margaritae margaritae & & & $\square 26,27$ & & & & & $\bigsqcup_{2-11}$ \\
\hline \multirow{14}{*}{ Miocene } & N. dutertrei & & & & & & & & \\
\hline & G. acostaensis & \multirow{3}{*}{\begin{tabular}{|l|}
$\square_{12,13}$ \\
$\square_{14}$ \\
$\square_{15}$
\end{tabular}} & & & & & & $\square 2,3$ & $\square 12-14$ \\
\hline & G. menardii & & & & & & & & \\
\hline & G. mayeri & & & & & & & & \\
\hline & G. ruber & & & & & & & & \\
\hline & G. fohsi robusta & \multirow{3}{*}{$\begin{array}{l}\square_{15} \\
\prod_{16}\end{array}$} & & & & & & & \\
\hline & G. fohsi lobata & & & & & \multirow{2}{*}{$\square^{3}$} & & & \\
\hline & G. fohsi fohsi & & & & & & & $\square 4$ & \\
\hline & G. fohsi peripheroronda & \multirow{6}{*}{ 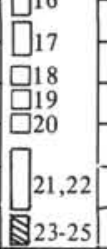 } & & & & \multirow{2}{*}{$\mathbb{E}_{4}$} & & & \\
\hline & P. glomerosa & & & & & & & & \\
\hline & G. insueta & & & & $\square$ & & & \$ 5 & \\
\hline & G, stainforthi & & & & 4 & $\mathbb{8} 4$ & & & \\
\hline & G. dissimilis & & & & & $\$ 5$ & & & \\
\hline & G. primordius & & & & $\square 5$ & & & & \\
\hline \multirow{5}{*}{ Oligocene } & G. kugleri & 電26-28 & & & & \multirow{2}{*}{$\begin{array}{r}\$ 6 \\
\$ 7 \\
\$ 8 \\
\end{array}$} & & $\$ 6$ & \\
\hline & G. cip. ciperoensis & & & & & & & & \\
\hline & G, opima opima & 专29.30 & & & & $\square 9$ & & & \\
\hline & G. ampliapertura & & & & & & & \& 7 & \\
\hline & G. chipolensis/H. micra & & & & & & & & \\
\hline \multirow{12}{*}{ Eocene } & G. cerroazulensis s.l. & & & & & & & & \\
\hline & G. semiinvoluta & & & & & & & & \\
\hline & T. rohri & & & & & & & & \\
\hline & O. beckmanni & & & & & & & & \\
\hline & G. lehneri & $1-43$ & & & & & & & \\
\hline & G. subcong. subconglobata & & & & & & & & \\
\hline & H. aragonensis & & & & & & & & \\
\hline & G. palmerae & & & & & & & & \\
\hline & G. aragonensis & & & & & & & & \\
\hline & G. formosa formosa & & & & & & & $\square 8$ & \\
\hline & G. subbotinae & & & & & & $\mathbb{8} 1$ & & \\
\hline & G, edgari & 娄 $5-7$ & & & $\mathrm{~A}$ A2 & $\square 10$ & 恖 2 & & \\
\hline & G. velascoensis & & & & & & 4 & & \\
\hline & G. pseudomenardii & & & & & & & & \\
\hline & G. pusilla pusilla & & & & & & \$ 6-8 & 舟9,10 & \\
\hline Paleocene & G. angulata & & & & & $\Pi_{10}$ & $\$ 9$ & $\mathbb{S}^{11}$ & \\
\hline & G. uncinata & & & & & & & & \\
\hline & G..trinidadensis & & & & & $\square 11$ & & & \\
\hline & G. pseudobulloides & 专 & & & & & & & \\
\hline & G. eugubina & & & & & & $\square 10$ & $\square 12$ & \\
\hline
\end{tabular}

Figure 1. Cenozoic sediments cored on Leg 15 in the Caribbean. Cores are represented by numbered rectangles. Empty rectangles represent calcareous cores lacking siliceous microfossils, hachuring indicates siliceous microfossils occurring in calcareous sediments, and filled rectangles represent cores with many siliceous and very few or no calcareous microfossils. 
Base of the Theocyrtis tuberosa Zone between Cores 30 and 31 (at approximately $270 \mathrm{~m}$ ).

The radiolarian assemblages in Cores 31 and 32 contain a high proportion of reworked specimens, so that it is difficult to recognize the bases of the Thyrsocyrtis bromia and Podocyrtis goetheana Zones.

Base of the Podocyrtis chalara Zone is probably in the lower part of Core 32 (at approximately $283 \mathrm{~m}$ ).

Base of the Podocyrtis mitra Zone between the bottom of Core 36 and the lower part of Core 37 (at approximately $317-330 \mathrm{~m}$ ).

Base of the Podocyrtis ampla Zone between Cores 38 and 39 (at $337-344 \mathrm{~m}$ ).

Base of the Thyrsocyrtis triancantha Zone is between Cores 42 and 43 (at approximately $376-381 \mathrm{~m}$ ).

Base of the Theocampe mongolfieri Zone is below (and evidently not far below) the bottom of Core 43 (at approximately $390 \mathrm{~m}$ ).

\section{Site 150}

Apart from a few "ghosts" of poorly preserved (probably zeolitized) skeletons in the catcher sample of Core 4 (from 95 to approximately $100 \mathrm{~m}$ below the sediment surface), no radiolarians were found in Cores 1 through 5 (from 49 to $114 \mathrm{~m}$ ). The catcher sample from Core 2 of Hole 150A (from $119-120 \mathrm{~m}$ ) contains rare, moderately preserved radiolarians (some dissolved, some infilled) which may be Paleocene in age. The cherts of Cores 6 through 8 (from 127 to approximately $142 \mathrm{~m}$ ) contain few to common, poorly preserved (silicified, dissolved, and altered) radiolarians of Cretaceous age. Soupy material from Core 7 contains few Cretaceous forms (Plate 4, Figures 13-15) and abundant, moderately well preserved radiolarians of the Buryella clinata Zone (Early Eocene)the latter evidently caved from higher in the hole. A small piece of gray mud from the catcher of Core 8 contains a few, moderately preserved (some dissolved and some infilled) Cretaceous skeletons. Radiolarians are rare, moderately well preserved in Section 1 of Core 9 (at approximately $150 \mathrm{~m}$ ), absent in Core 10 (at 159 to approximately $160 \mathrm{~m}$ ), and common and poorly preserved (somewhat dissolved and silicified) in Core 12 (at $177-180 \mathrm{~m})$.

\section{Site 151}

No radiolarians are present in Cores 1 through 3 (from 61 to $190 \mathrm{~m}$ below the sea floor). Core 4 (at 237 to approximately $240 \mathrm{~m}$ ) contains abundant, moderately well preserved (somewhat dissolved) radiolarians, those in the top of the core belonging to the Dorcadospyris alata Zone, and those lower in the core belonging to the Calocycletta virginis Zone-the Calocycletta costata Zone being either missing or included in the unsampled $40 \mathrm{~cm}$ below 151-4-1 $(28-30 \mathrm{~cm})$. Radiolarians are also abundant and moderately well preserved in Core 5 (at 302 to approximately $305 \mathrm{~m}$ ), most of the core belonging in the $C$. virginis Zone and its base in the Lychnocanoma elongata Zone. Cores 6 through 8 (at 311 to approximately $330 \mathrm{~m}$ ) contain abundant, well preserved assemblages of the Dorcadospyris ateuchus Zone. The samples examined from Cores 7 and 8 contain a few reworked, Middle Eocene forms. Core 9 (at
339 to approximately $341 \mathrm{~m}$ ) contains rare, poorly preserved (somewhat dissolved) radiolarians including Pterocodon ampla as well as some apparently younger forms. No radiolarians were found in Cores 10 through 12 (at 348-376 m).

\section{Site 152}

Radiolarians are abundant and well preserved in Core 1 (at 153 to approximately $156 \mathrm{~m}$ below the sediment surface). They are rare to common, poorly to well preserved, in Cores 2 through 9 (from 162 to approximately $230 \mathrm{~m}$ ). Core 1 and the upper part of Core 2 (to approximately $165 \mathrm{~m}$ ) evidently belong in the Bekoma bidarfensis Zone (though the zonal marker is absent); the remainder of Core 2, Core 3, and the upper part of Core 4 (to approximately $185 \mathrm{~m}$ ) belong either in the $B$. bidarfensis Zone or in the underlying "unzoned interval" of the Leg 10 Initial Report; and the radiolarian samples below this (through Core 9) are apparently in that "unzoned interval."

No radiolarians are present in Core 10 (at approximately $239 \mathrm{~m}$ ). In the Cretaceous Cores 12 to 22 (from 257 to approximately $466 \mathrm{~m}$ ) they are generally absent or present as rare to few, poorly preserved (silicified) specimens.

\section{Site 153}

Cores 1 through 4 (to $309 \mathrm{~m}$ below the sediment surface) are barren of radiolarians. The lower part of Core 5 and Core 6 (from approximately 408 to $415 \mathrm{~m}$ ) contain common, moderately to well preserved (somewhat dissolved) radiolarians of the Calocycletta virginis Zone. Core 7 (at 499 to approximately $501 \mathrm{~m}$ ) contains abundant, moderately to well preserved radiolarians of the Theocyrtis tuberosa Zone, with some reworked Middle Eocene forms.

Cores 9 to 11 (from 586 to approximately $604 \mathrm{~m}$ ) contain rare to few, poorly to moderately preserved (somewhat dissolved) radiolarians corresponding to the Paleocene unzoned interval of the Leg 10 Initial Report. The Cretaceous Cores 12 to 18 (from 609 to approximately $754 \mathrm{~m}$ ) contain no radiolarians.

\section{SPECIES OCCURRENCES AND BIOSTRATIGRAPHIC RESULTS}

In Tables 1 to 8 , which present the information on abundances of radiolarians in each sample examined from Sites $146,149,151,152$, and 153 , the following abbreviations are used: A (abundant), C (common), R (rare), + (very rare), ? (doubtful identification), ! (believed to be reworked from older sediments, or caved from younger), - (looked for, and not found), and X (not found, but absence may be due to some secondary factor such as solution, excessive sieving of the assemblage, etc.)

The results from Site 150 are not suitable for tabulation and are as follows.

\section{Radiolarians From Site 150}

The catcher sample from Core 150-2A contains rare, moderately preserved radiolarians, and that from Core 150-7 contains rare, poorly preserved radiolarians-neither of these assemblages being sufficient to merit tabulating. 
TABLE 1

Radiolarians from Site 146 (Upper Section)

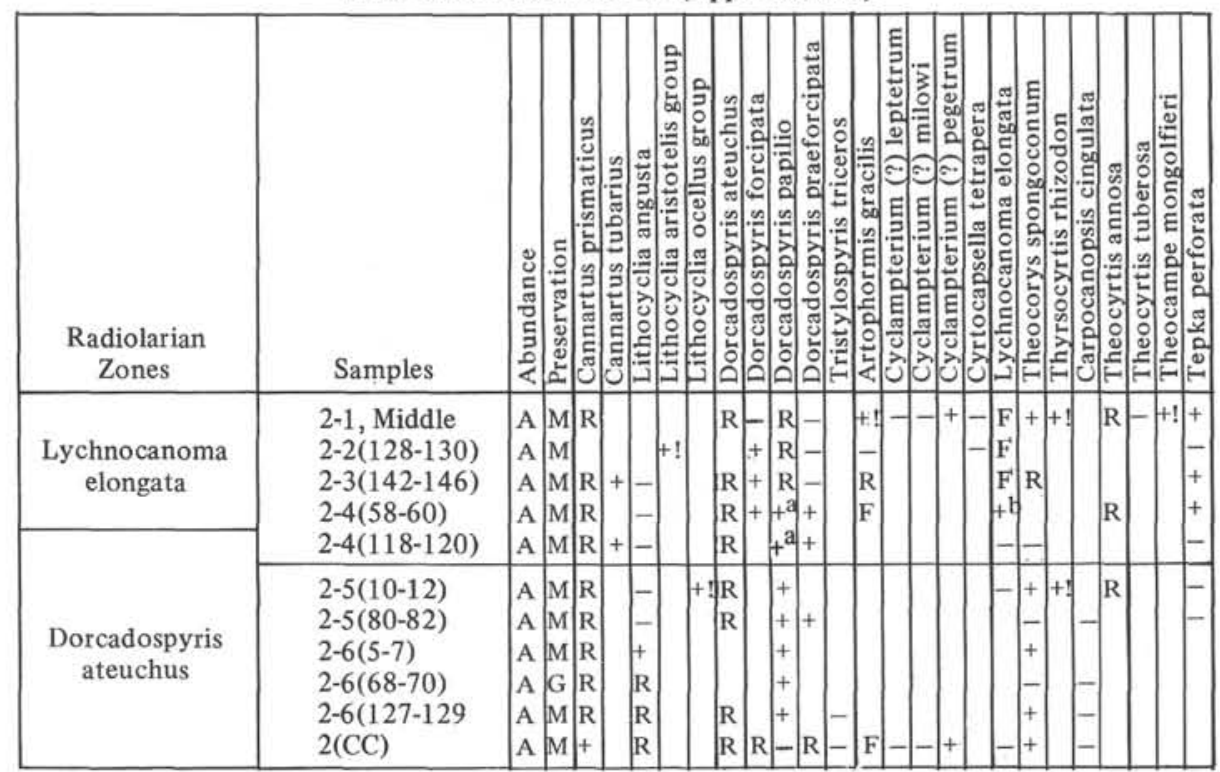

${ }^{\mathrm{a}}$ In these samples, specimens of Dorcadospyris papilio are outnumbered by specimens of the ancestral form, in which the two strong feet diverge at $180^{\circ}$ or less.

${ }^{\mathrm{b}}$ Samples below this level contain specimens (Plate 1, Figure 1) similar in structure to Lychnocanoma elongata, except that the feet are three in number and somewhat shorter, less robust and straighter than in that species. In rare specimens, one of the feet appears to have atrophied and the other two to be situated approximately $150^{\circ}$ apart.

The soupy, fluid sediment from Core 150-7 contains common, well preserved radiolarians of the Buryella clinata Zone, evidently caved from higher in the hole. Species present, and their abundances, are

Amphisphaera minor (R)

Astrosphaerin sp. E (R)

Axoprunum pierinae group (R)

Stylosphaera coronata coronata $(\mathrm{F})$

Thecosphaerella larnacium $(\mathrm{F})$

Thecosphaerella ptomatus $(\mathrm{F})$

Thecosphaerella rotunda $(\mathrm{R})$

Heliostylus $\mathrm{sp}(\mathrm{p})(\mathrm{R})$

Amphicraspedum murrayanum (+)

Amphicraspedum prolixum (R)

Amphicraspedum prolixum group (R)

Spongodiscus quartus quartus (R)

Stylotrochus nitidus $(+)$

Dendrospyris fragoides $(\mathrm{R})$

Dictyospyris discus $(\mathrm{R})$

Giraffospyris lata (R)

Rhabdolithis pipa $(+)$

Buryella clinata $(\mathrm{R})$

Buryella tetradica $(\mathrm{F})$

Calocycloma castum (R)

Lamptonium fabaeforme chaunothorax (R)

Lamptonium fabaeforme fabaeforme (R)

Lamptonium pennatum $(\mathrm{R})$

Lithochytris archaea (F) (Plate 5, Figure 1)

Lychnocanoma babylonis group (F)

Phormocyrtis cubensis (R)

Phormocyrtis striata exquisita $(\mathrm{F})$

Phormocyrtis striata striata $(\mathrm{F})$

Phormocyrtis turgida (+)

Pterocodon tenellus ( $\mathrm{R})$

Theocorys (?) phyzella (R)

Theocotyle alpha $(\mathrm{R})$

Thyrsocyrtis hirsuta hirsuta (R)

Thyrsocyrtis hirsuta tensa $(+)$

\section{Podocyrtis papalis $(\mathrm{F})$}

Amphiternis sp. cf. Stichomitra alamedaensis (R)

A few apparently Cretaceous specimens (e.g., Plate 4, Figures 13-15) occur together with this Early Eocene assemblage.

Table 9 presents a stratigraphically arranged listing of earliest and latest occurrences of radiolarian taxa in a format similar to that used in our report on radiolarians from Leg 10 (Sanfilippo and Riedel, 1973). The order of the listing is based as far as practicable on Leg 15 results, but with some account being taken of data from earlier legs. In the column with the names of taxa, $\mathrm{T}$ indicates the top of the range of a taxon, B its bottom, and an arrow an evolutionary transition. The abbreviations $e, m$, and $m-e$ indicate evolutionary, morphotypic, and morphotypicevolutionary limits, respectively. In the body of the table are given the pairs of core-sections between which an event occurred, followed by the depth in meters below the sediment surface in parentheses. The degree of reliability (for purposes of correlation) of each event at each site is indicated by the letters $\mathrm{P}, \mathrm{M}$, and $\mathrm{G}$ (for poor, moderate, and good).

Some parts of this list of radiolarian events are well supported by Leg 15 data, but the following are not. Events indicated as occurring above the top of Core 151-4 are arranged in the order indicated in our Leg 10 report (which depended partly on results from earlier legs). Between core-sections 149-30(CC) and 149-32-2, the order of events in the Leg 15 material is obscured by extensive reworking, and therefore the order indicated in the Leg 10 report is followed. There is a considerable period of time between Cores $149-43$ and $152-1$, insufficiently represented in the Leg 15 material, and here again the Leg 10 order is followed. And as can be seen from the tabulations of occurrences of species in the lower cores from Sites 146 and 152 , there is little reliable evidence for the stratigraphic order of the events indicated as occurring below Core 152-4-these are here listed only tentatively, pending recovery of better material of this age. 
TABLE 2

Radiolarians from Site 146 (Lower Section)

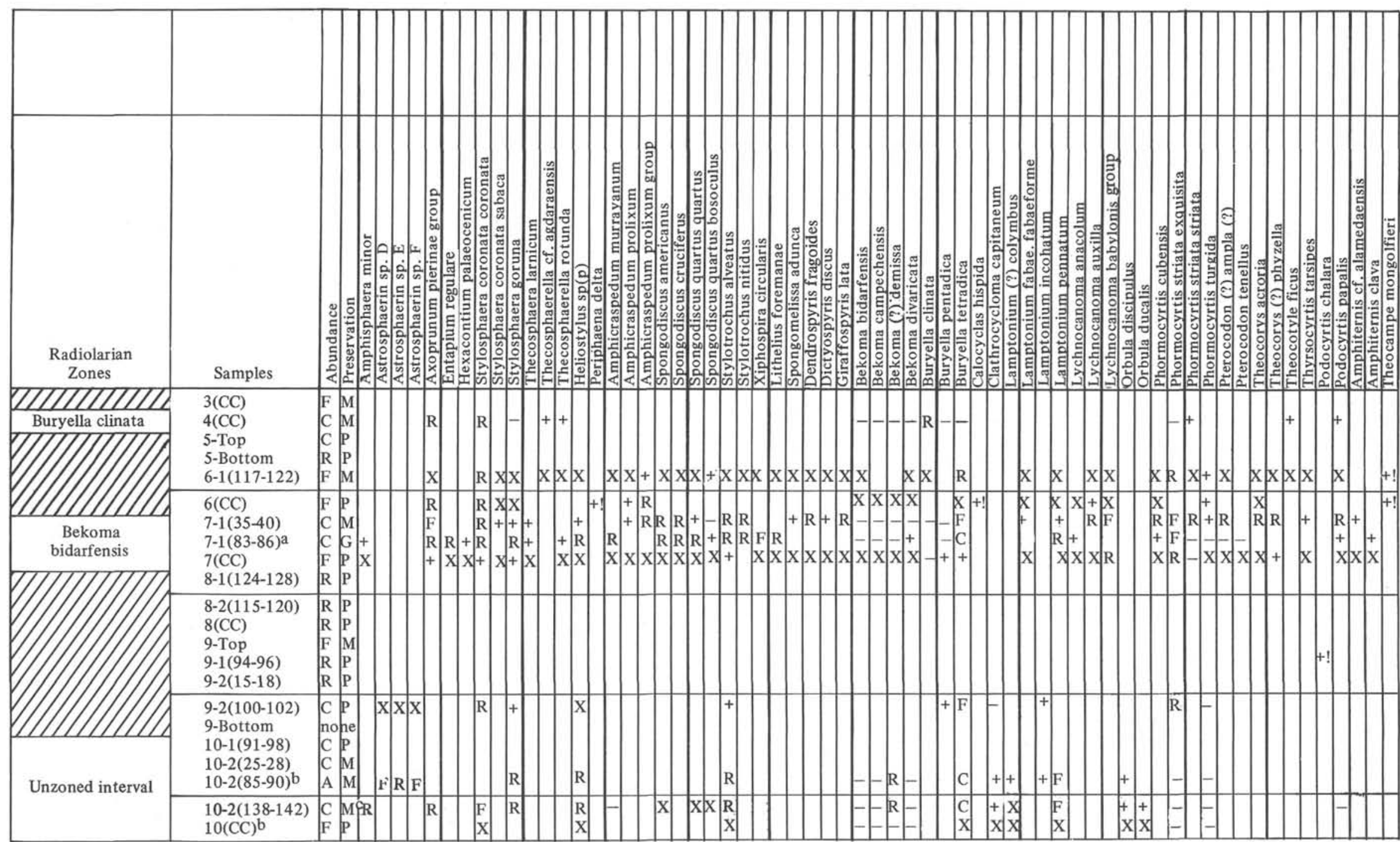

${ }^{a}$ This assemblage includes a well preserved saturnalin with spiny ring and concentrically zoned cortical shell (Plate 1, Figure 2).

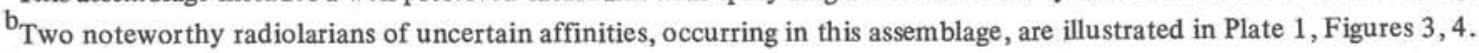

${ }^{\mathrm{c}}$ Some of the radiolarians in this assemblage are completely pyritized (Plate 1 , Figures 5,6 ). 
TABLE 3

Radiolarians from Site 149 (Upper Section)

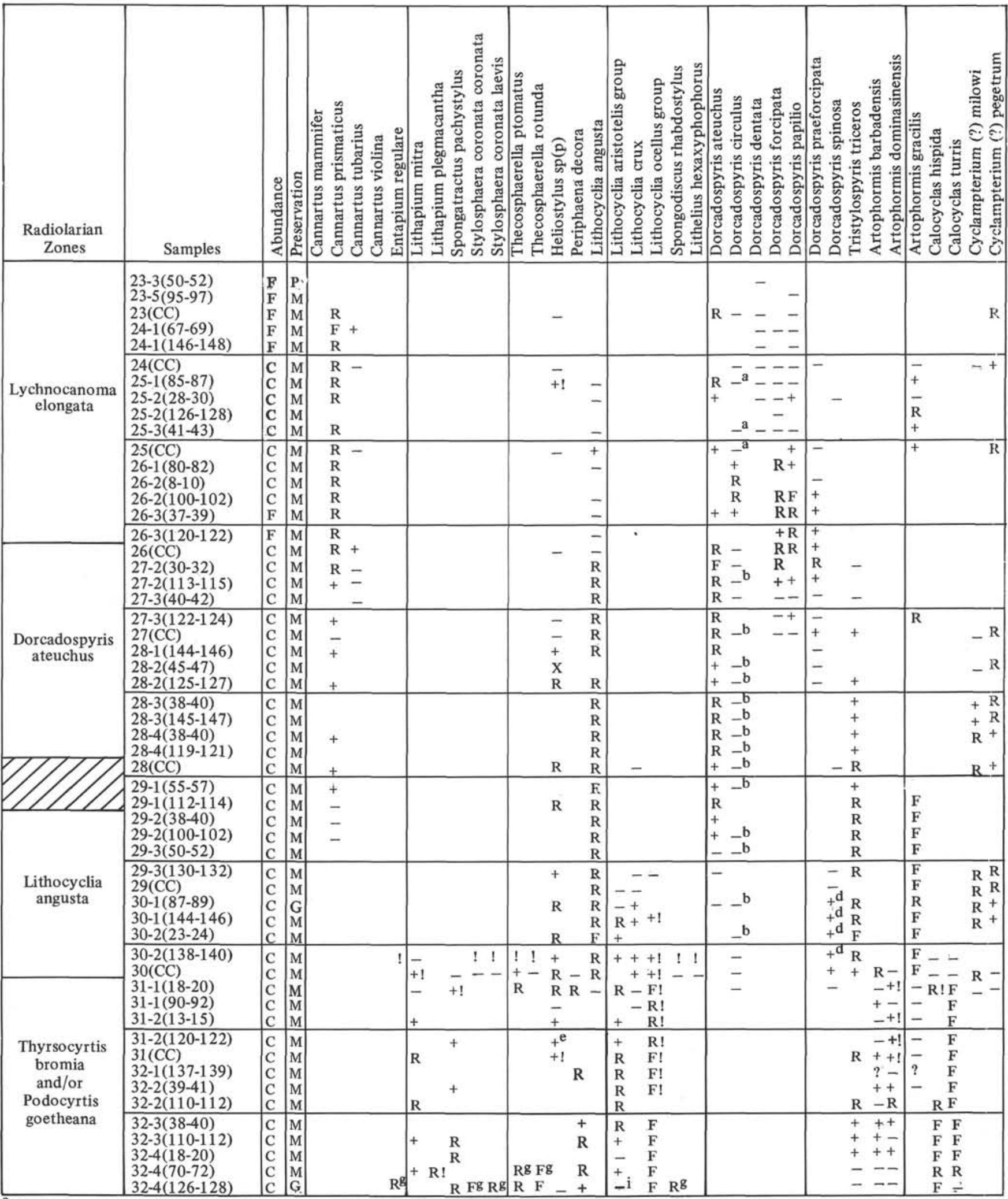

${ }^{a}$ Above the range of Dorcadospyris circulus, and in 149-28-4, 119-121 cm, assemblages include very rare to rare specimens of a rather similar form with a very small apical horn, and accessory spinules on the two joined feet (Plate 1, Figure 7).

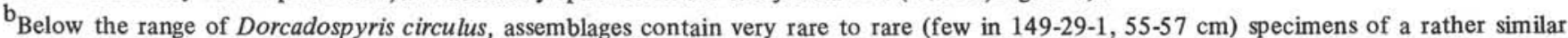
form with two feet joined but with the apical horn very small or absent, and no accessory spinules on the feet (Plate 1, Figures 9, 10).

${ }^{c}$ These samples contain specimens resembling Theocorys spongoconum, but with the abdominal wall being a simple lattice, not spongy (Plate 2 , Figure 1).

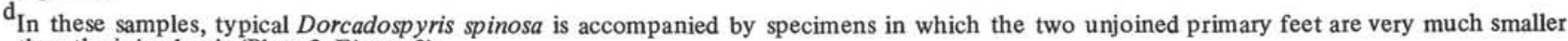
than the joined pair (Plate 2, Figure 2).

${ }^{\mathrm{e}}$ Specimens of Heliostylus sp(p). at and above this level have large pores on the cortical shell (Plate 2, Figure 3), while specimens from Cores 710 
TABLE 3 - Continued

\begin{tabular}{|c|c|c|c|c|c|c|c|c|c|}
\hline 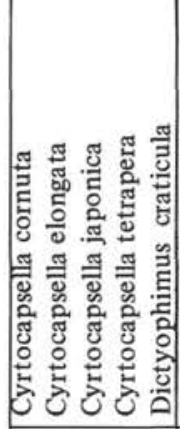 & 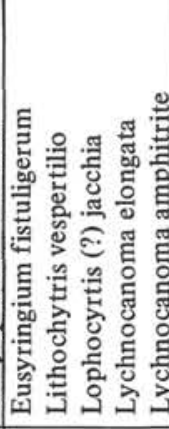 & 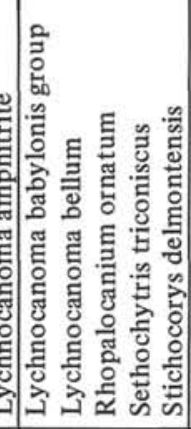 & 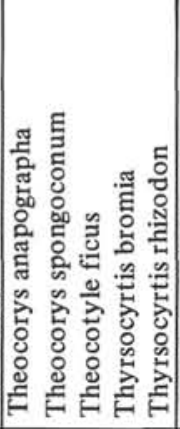 & 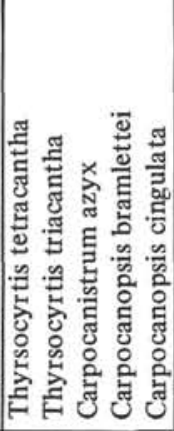 & 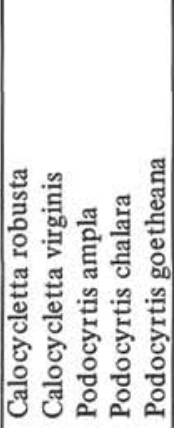 & 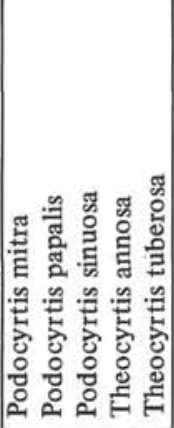 & 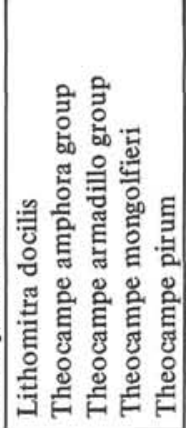 & 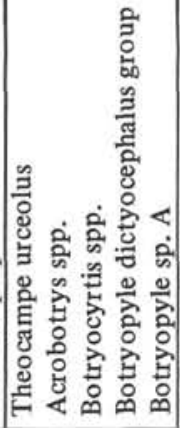 & 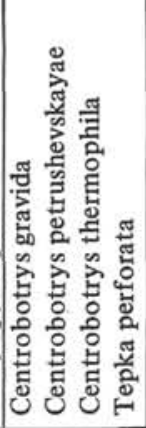 \\
\hline $\begin{array}{r}---\frac{-}{\mathrm{R}} \\
--\frac{-}{-} \\
-\end{array}$ & $\begin{array}{l}\mathrm{R} \\
\mathrm{F} \\
\mathrm{R}\end{array}$ & & $\begin{array}{l}\mathrm{R} \\
+ \\
\mathrm{R}\end{array}$ & $\begin{array}{l}- \\
- \\
- \\
-\end{array}$ & $\begin{array}{l}\mathrm{C} \\
\mathrm{C} \\
\mathrm{C} \\
\mathrm{C}\end{array}$ & $\begin{array}{l}\mathrm{R} \\
\mathrm{R} \\
\mathrm{R}\end{array}$ & & $\mathrm{X}$ & $\begin{array}{r}\mathrm{R} \\
\mathrm{X} \times \mathrm{R} \\
\mathrm{R} \\
\mathrm{R}\end{array}$ \\
\hline - & $\begin{array}{l}\mathrm{R} \\
\mathrm{R} \\
\mathrm{R} \\
\mathrm{R} \\
\end{array}$ & & $\begin{array}{l}+ \\
+\end{array}$ & $\begin{array}{r}-\frac{-}{R} \\
R \\
R \\
\end{array}$ & $\begin{array}{l}\mathrm{C}- \\
\mathrm{C} \\
\mathrm{C} \\
\mathrm{C}\end{array}$ & $\mathrm{R}$ & & $\begin{array}{l}\mathrm{X} \\
\mathrm{X}\end{array}$ & $\begin{array}{r}\mathrm{XX \textrm {R }} \\
\mathrm{XX}+ \\
+ \\
+ \\
\end{array}$ \\
\hline & $\begin{array}{l}\mathrm{R} \\
\mathrm{F} \\
\mathrm{R} \\
\mathrm{R} \\
+ \\
\end{array}$ & & $\mathrm{R}$ & $\begin{array}{l}+ \\
\mathrm{R}\end{array}$ & $\begin{array}{l}\text { C } \\
\text { C } \\
\text { C } \\
\text { C } \\
\end{array}$ & $\begin{array}{l}+ \\
\mathrm{R} \\
\mathrm{R} \\
\mathrm{R}\end{array}$ & & $\begin{array}{r}\mathrm{X} \\
\mathrm{R} \\
+\quad+\mathrm{R} \\
\end{array}$ & $\begin{array}{l}\mathrm{X} \\
\mathrm{X} \\
\mathrm{X} \mathrm{X}^{+} \\
\end{array}$ \\
\hline & $\begin{array}{l}+ \\
- \\
-\end{array}$ & & & $\begin{array}{l}\mathrm{R} \\
- \\
-\end{array}$ & $\begin{array}{l}\mathrm{C} \\
\mathrm{F} \\
\mathrm{R}\end{array}$ & $\begin{array}{l}\mathrm{R} \\
\mathrm{R} \\
+ \\
\mathrm{R}\end{array}$ & & $\begin{array}{ll}\mathrm{X} & \mathrm{X} \\
\mathrm{X} & \mathrm{X} \\
\end{array}$ & $\mathrm{XX}^{+} \mathrm{x}_{-}^{+}$ \\
\hline & & & $\begin{array}{l}\mathrm{R} \\
\mathrm{R}\end{array}$ & - & $\begin{array}{l}\mathrm{R} \\
- \\
- \\
-\end{array}$ & $\begin{array}{l}\mathrm{F} \\
\mathrm{R} \\
\mathrm{R} \\
+ \\
\mathrm{F}\end{array}$ & $+!$ & \begin{tabular}{|r}
- \\
--+ \\
$\mathrm{R}$
\end{tabular} & $\begin{array}{r}\mathrm{X} X \\
-+\mathrm{R} \\
\mathrm{X} X \\
\mathrm{R} \\
\mathrm{R}\end{array}$ \\
\hline & & & - & & & $\begin{array}{l}+ \\
+ \\
\mathrm{R} \\
\mathrm{R} \\
\mathrm{R}\end{array}$ & - & --- & $\begin{array}{c}+ \\
\mathrm{R}+ \\
---\end{array}$ \\
\hline & & & $-c$ & & & - & $\begin{array}{l}- \\
\overline{-} \\
\bar{R}\end{array}$ & $--F-$ & $-F--$ \\
\hline & & - & $\begin{array}{l}+\mathrm{c} \\
-\mathrm{c}\end{array}$ & & & -- & $\begin{array}{l}\mathrm{R} \\
\mathrm{R}-\ldots\end{array}$ & $\begin{array}{r}--\mathrm{R} \\
---\mathrm{F}-\end{array}$ & $\begin{array}{l}-\bar{F}-- \\
-\mathrm{R}\end{array}$ \\
\hline $\begin{array}{r}! \\
-\end{array}$ & $\begin{array}{rrr}- & - & \\
-\bar{R} & \overline{\mathrm{R}} & \overline{\mathrm{R}} \\
+! \mathrm{R} ! & \mathrm{R} !+ & \\
\mathrm{R} ! & - & +\end{array}$ & \begin{tabular}{l|l}
- & $--!-$ \\
$R+$ & \\
&
\end{tabular} & $\begin{array}{|rrr|}!-1 & - \\
\mathrm{R} & \bar{F} \bar{F} \\
& \text { F C } \\
& \text { R F } \\
\end{array}$ & $\begin{array}{l}-- \\
-+! \\
F \\
F ! \\
F\end{array}$ & $\begin{aligned} &- \\
&-- \\
&+! \mathrm{F} !- \\
& \mathrm{R} !- \\
& \mathrm{R} !-\end{aligned}$ & 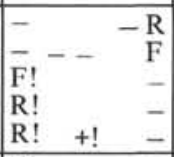 & 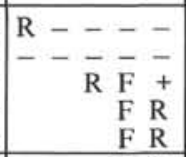 & $\begin{array}{r}---\mathrm{F}- \\
--\overline{\mathrm{R}}- \\
-\frac{-}{2}\end{array}$ & $\begin{array}{l}\text { F- - } \\
- \\
-\end{array}$ \\
\hline & $\begin{array}{lll}\mathrm{R} ! & + & \\
\mathrm{R} ! \mathrm{R} !+ & \mathrm{R} \\
\mathrm{R} ! \mathrm{R} ! & - & \mathrm{R} \\
\mathrm{R} \mathrm{R} !- & \mathrm{R} \\
\mathrm{R} \mathrm{R} !- & + \\
\end{array}$ & 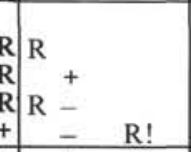 & $\begin{array}{ll}\mathrm{F} & \mathrm{F} \\
\mathrm{F} & \mathrm{F} \\
& \mathrm{F} \\
\mathrm{R} & \mathrm{C} \\
& \mathrm{C} \\
\end{array}$ & $\begin{array}{llll}F & F ! & R \\
F & F ! & R \\
F & F ! & F \\
C & F & F \\
R & F & F \\
\end{array}$ & $\begin{array}{r}\mathrm{R} !+ \\
++ \\
\mathrm{R}- \\
\mathrm{R}- \\
\mathrm{R} ! \mathrm{R}-\end{array}$ & $\begin{array}{ll}\mathrm{F} ! & - \\
+! & - \\
\mathrm{R} ! \mathrm{R} ! \mathrm{R} ! & - \\
\mathrm{R} ! \mathrm{R} ! \mathrm{R} ! & - \\
\mathrm{F} ! & - \\
\end{array}$ & \begin{tabular}{|ll} 
F R \\
F R \\
F $\mathrm{R}$ \\
C R \\
F R \\
\end{tabular} & $\begin{array}{l}\mathrm{R}- \\
-- \\
\overline{\mathrm{R}}-\end{array}$ & \\
\hline $\mathrm{Rg}$ & 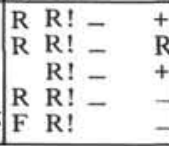 & \begin{tabular}{l|ll}
+ & + & +1 \\
$\mathrm{R}$ & $\mathrm{R}-$ \\
+ & $\mathrm{R}+\mathrm{R} !$ \\
& $\mathrm{R}$ \\
$-\mathrm{Rg}+\mathrm{R}$ & $\mathrm{R} !$
\end{tabular} & $\begin{array}{rr}\mathrm{C} \\
\mathrm{R} \\
\mathrm{R} \\
\mathrm{F} \\
\mathrm{R} \\
\mathrm{R} !^{+} \mathrm{F}^{\mathrm{F}}\end{array}$ & $\begin{array}{lll}F & F & F \\
F & F & F \\
R & F & F \\
-F & - \\
-F & -\end{array}$ & $\begin{array}{r}\mathrm{R}- \\
+! \mathrm{R}- \\
\mathrm{R} \\
\mathrm{F}+ \\
\mathrm{F}\end{array}$ & 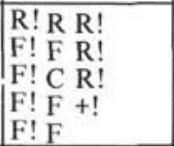 & 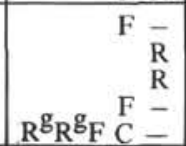 & $\begin{array}{l}\mathrm{R}- \\
- \\
-\end{array}$ & \\
\hline
\end{tabular}

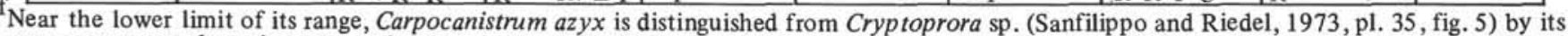
more numerous thoracic pores and more pronounced stricture at the base of the thorax. The cephalis is usually overgrown by the proximal thoracic wall, but specimens in which this is corroded show a simple, subspherical cephalis (Plate 2, Figure 4), in contrast to the original description.

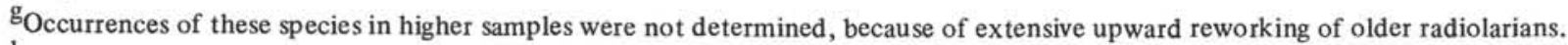

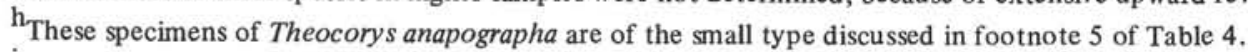

${ }^{i}$ Below the range of the forms tabulated as members of the Lithocyclia aristotelis group occurs a form (Plate 2, Figures 5,6) with the peripheral spongy zone divided into several arms terminating in spines, and the phacoid cortical shell so delicate that it is rarely preserved. This form may be ancestral to the $L$. aristotelis group, or an evolutionary off shoot near its origin. In the same samples occur members of the $L$. ocellus group with some structural similarity, in that the zone immediately beyond the cortical shell shows pronounced concentric zonation, and the structure beyond that is irregularly spongy (Plate 2, Figures 7,8).

${ }^{\mathrm{j}}$ The existence of a period of time between the latest occurrence of Theocyrtis tuberosa and the earliest $T$, annosa, indicated in the tabulations of radiolarians at Sites 94 and 95 of DSDP Leg 10 (Sanfilippo and Riedel, in press) is confirmed by these results from Site 149. 
TABLE 4

Radiolarians from Site 149 (Lower Section)

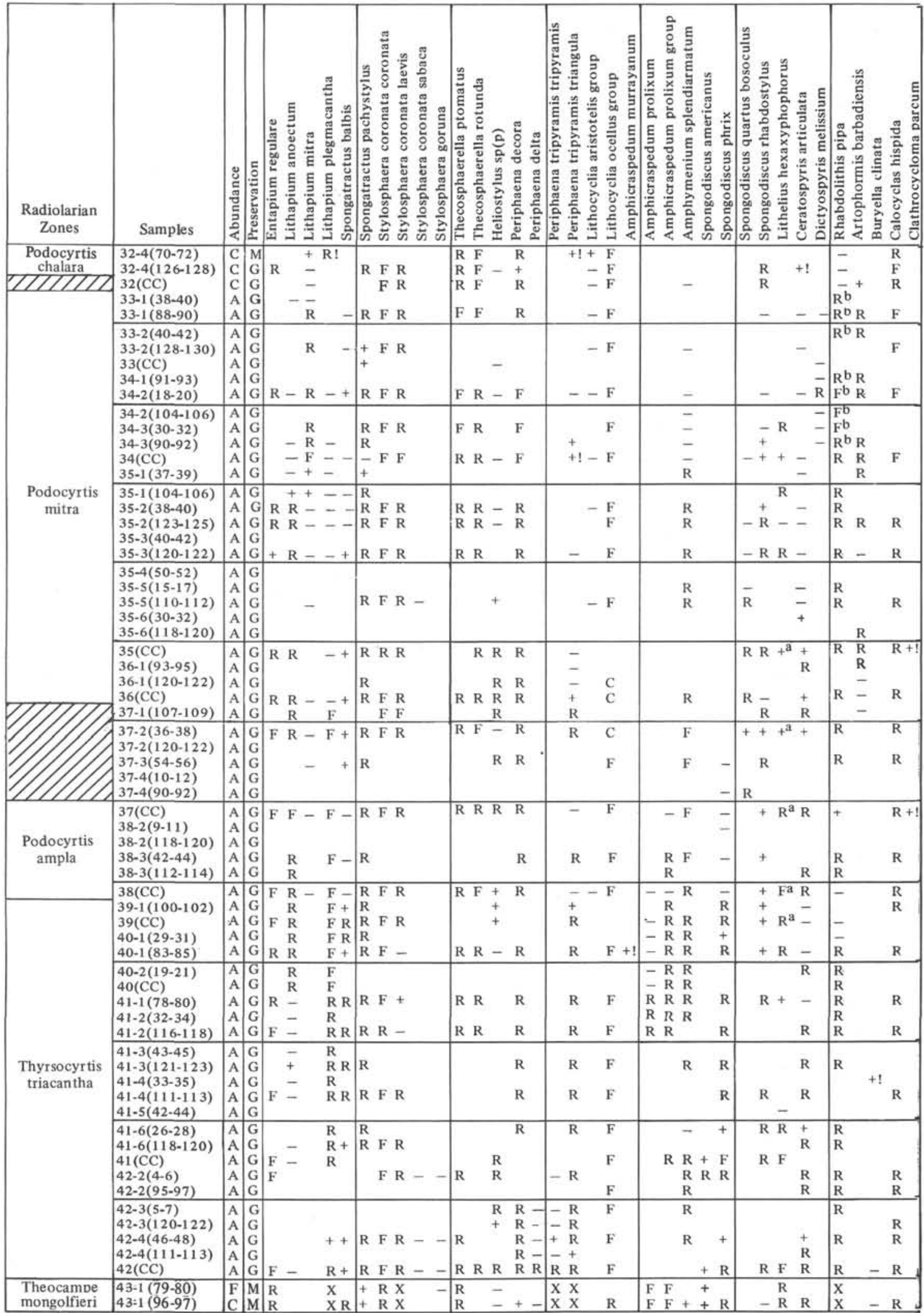

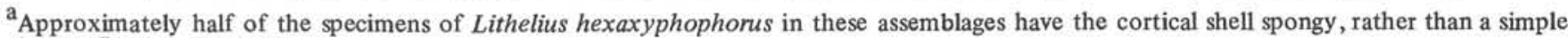
lattice (Plate 3, Figure 6).

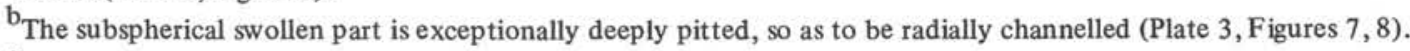

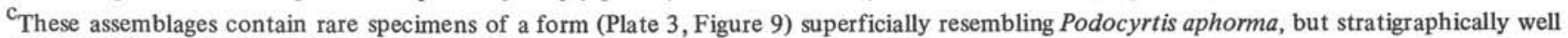
separated from the range of that earlier species. 
TABLE 4 - Continued

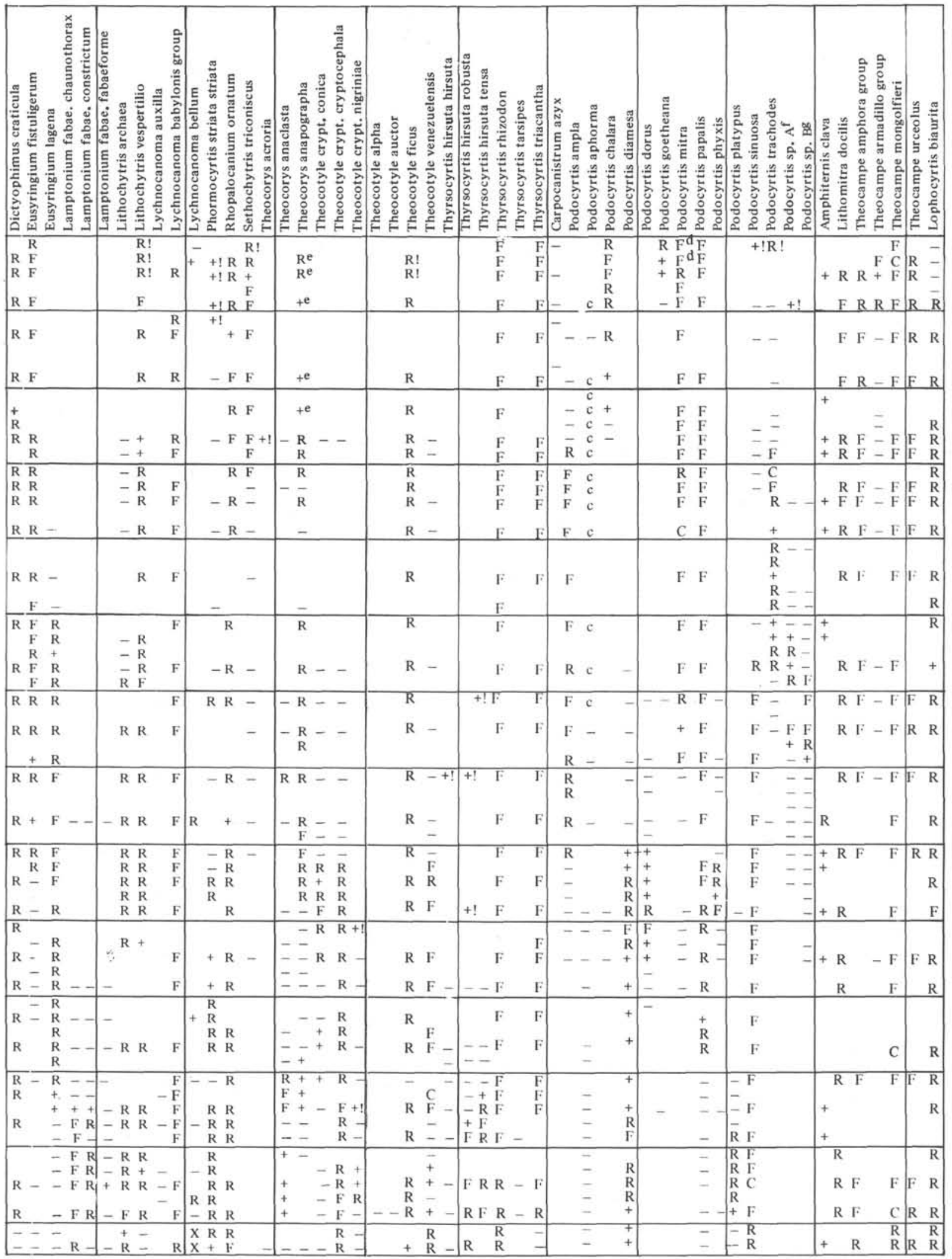

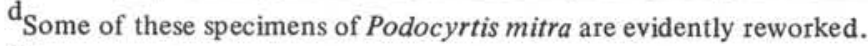

'These assemblages contain rare specimens of a small, hyaline, late form of Theocorys anapographa with very few pores (Plate 3, Figure 11).

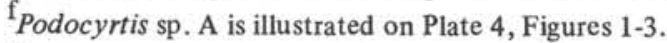

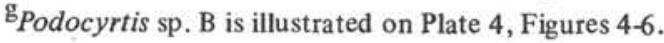




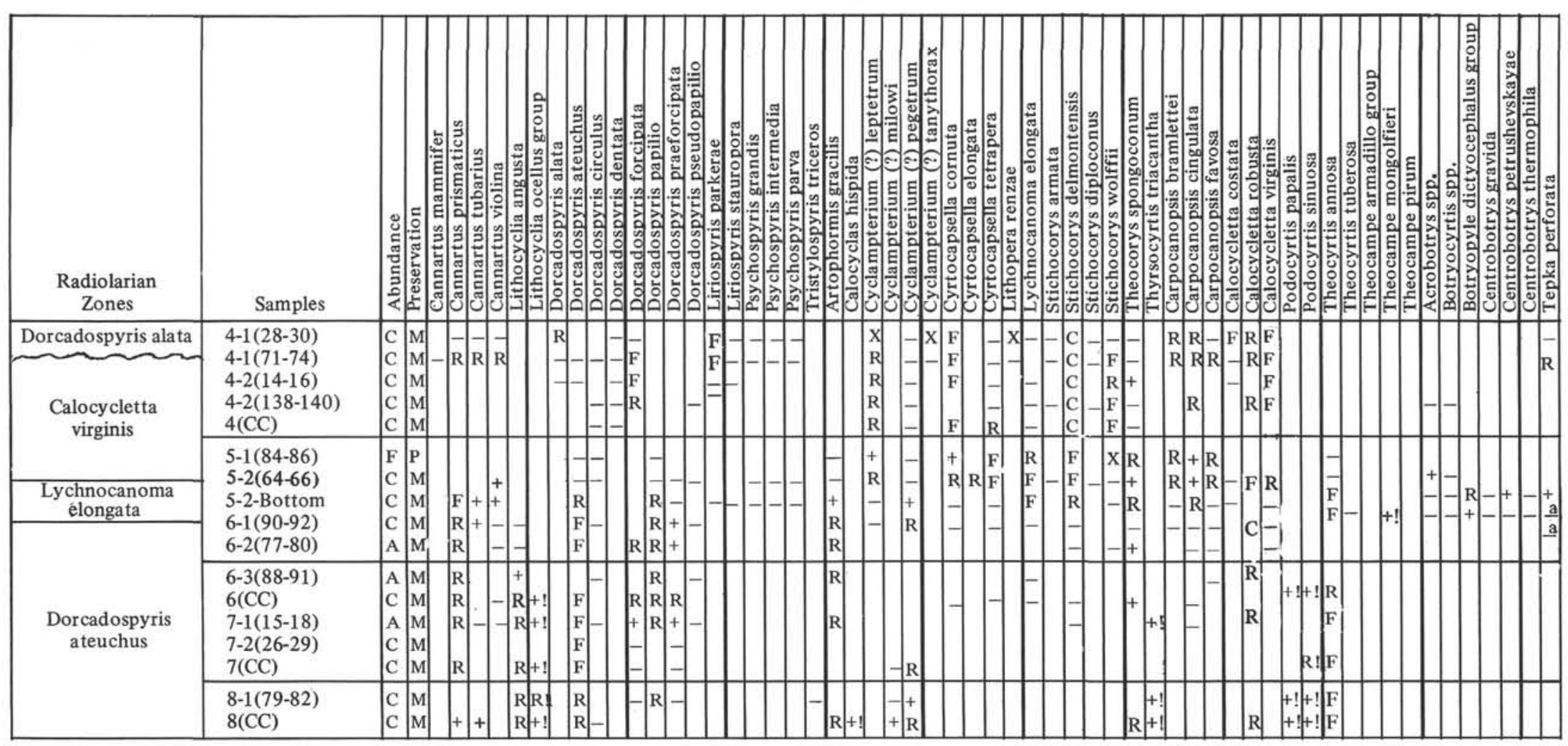

${ }^{\mathrm{a}}$ These samples contain a species of Tepka in which the perforated plate is represented only by a series of radii surrounding the central ring (Plate 5, Figures 2,3). 
TABLE 6

Radiolarians from Site 152

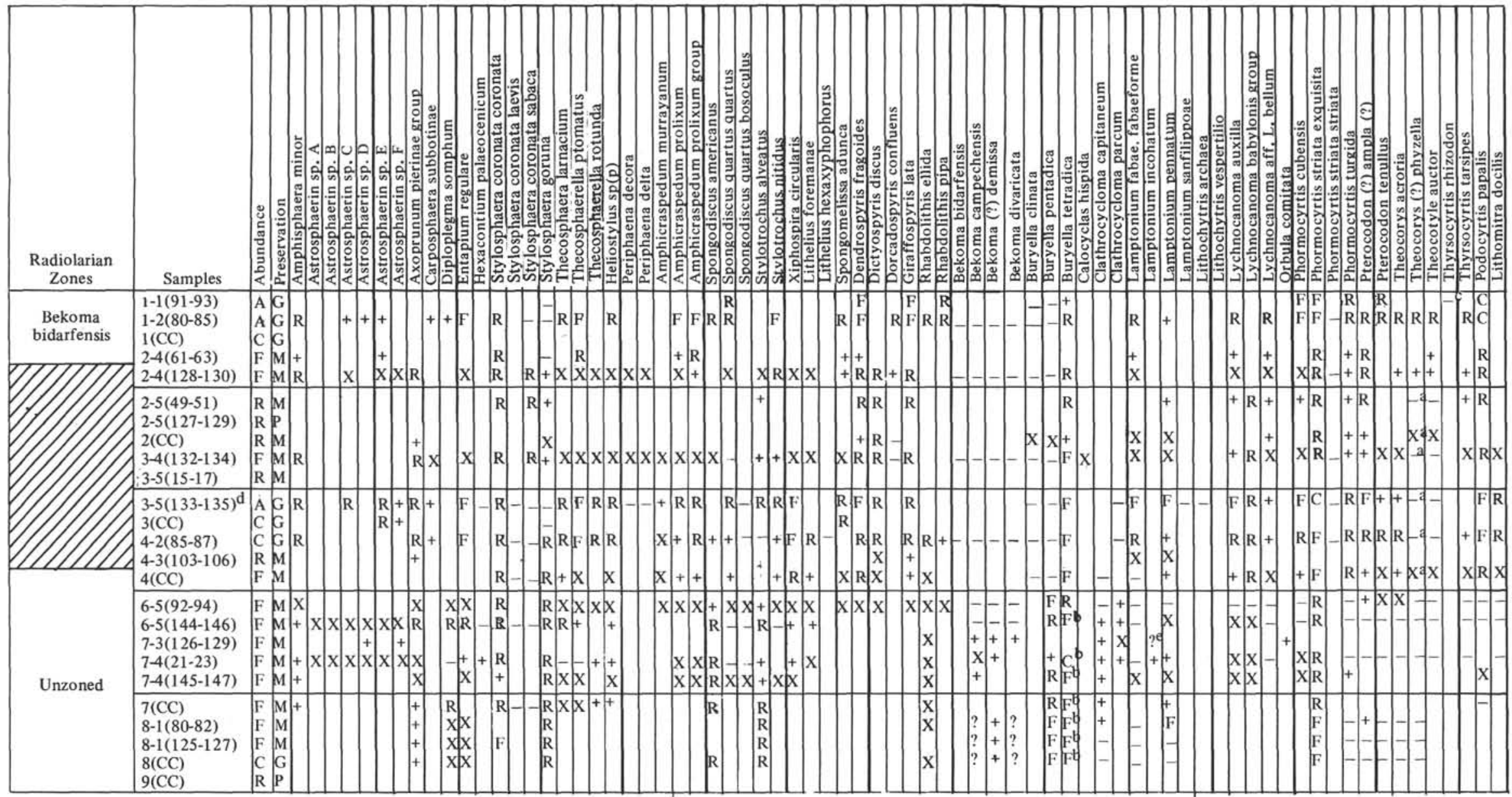

Footnotes to Table 6

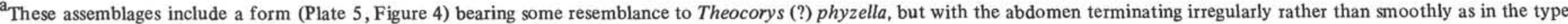
specimen.

${ }^{\mathrm{b}}$ Specimens of Buryella tetradica in these assemblages commonly have the fourth (downwardly tapering) segment subdivided rather than simple (Plate 5, Figure 5),

${ }^{c}$ This assemblage contains a form apparently ancestral to Thyrsocyrtis rhizodon, with lamellar triangular feet tending to be cleft distally (Plate 5, Figures 6,7 ).

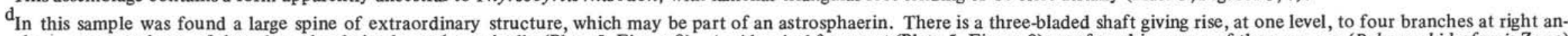
gles - some, at least, of these branches being looped terminally (Plate 5, Figure 8). An identical fragment (Plate 5, Figure 9) was found in a core of the same age (Bekoma bidarfensis Zone) collected by C. D. Hollister of Woods Hole Oceanographic Institution - Chain 100, Core 80 , taken in the tropical Pacific at lat. $08^{\circ} 18.8^{\prime} \mathrm{S}, 1$ long. $168^{\circ} 32.3^{\prime} \mathrm{W}$, in $4732 \mathrm{~m}$ of water.

$\mathrm{e}_{\mathrm{R}}$ are specimens in this assemblage resemble Lamptonium (?) incohatum, but have a delicate abdomen of large meshes (Plate 5, Figure 10), foreshadowed in the original description of the species (Foreman, 1973). 


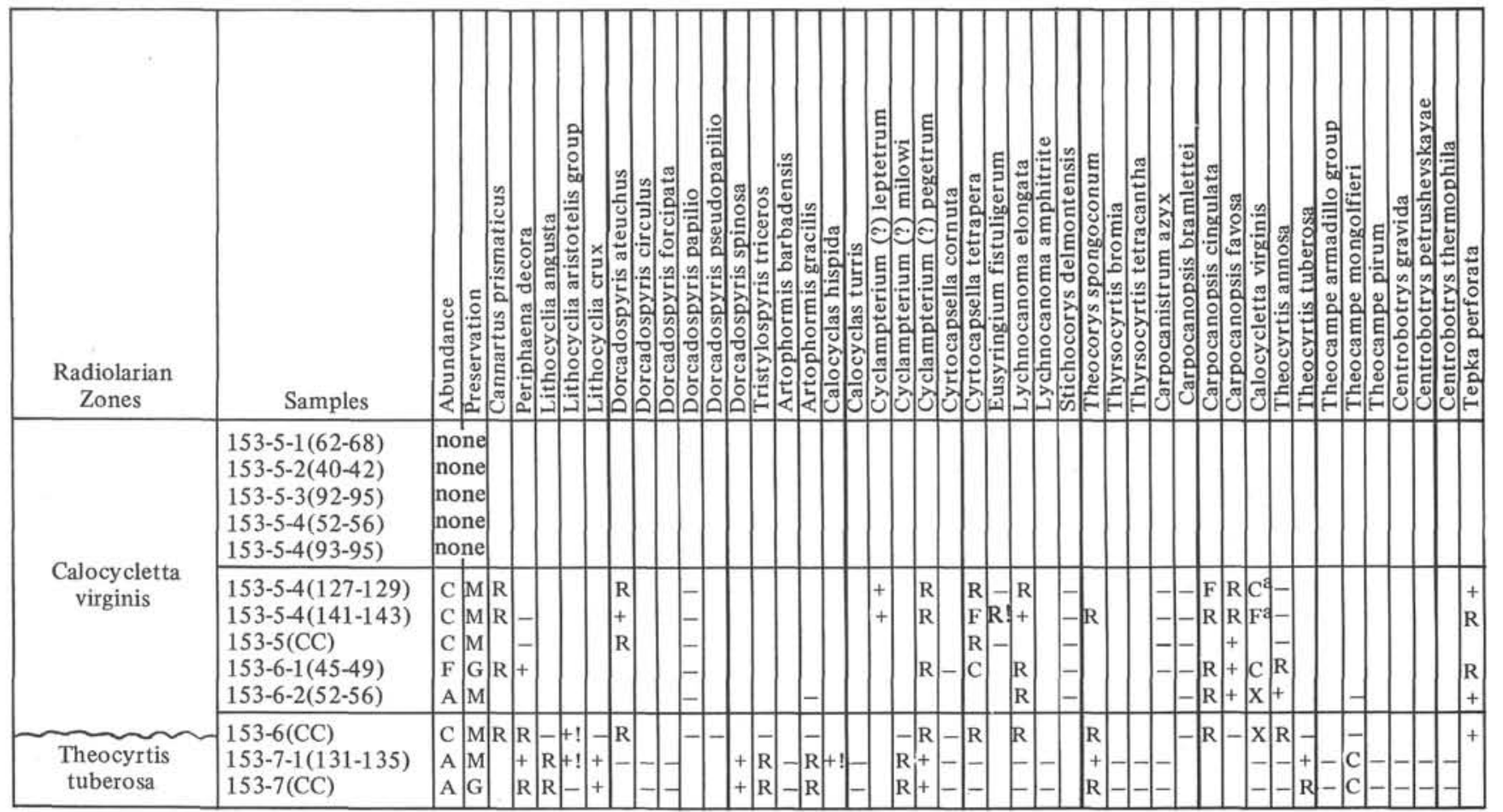

${ }^{\mathrm{a}}$ These assemblages contain specimens with shovel-shaped feet, as illustrated by Riedel and Sanfilippo (1970, pl. 14, fig. 11; 1971, pl. 8, fig. 12), and described as the new species Calocycletta serrata by Moore (1972).

This list of events differs slightly from those that we have presented previously, in that for some taxa both morphotypic and evolutionary limits are given (instead of only one of these). A "top" and a "bottom" is indicated for each included taxon, except for the following (listed in alphabetical order of species or subspecies) in which the evidence was insufficient.

"Tops" are not listed for -

Lychnocanoma sp. aff. L. bellum

Stylosphaera coronata coronata

Lithelius hexaxyphophorus

Stylosphaera coronata laevis

Centrobotrys petrushevskayae

Thecosphaerella ptomatus

Thecosphaerella rotunda

Diploplegma somphum

Carposphaera subbotinae

"Bottoms" are not listed for -

Lychnocanoma bellum

Spongodiscus quartus bosoculus

Theocotyle cryptocephala nigriniae

Figure 2 is a range-chart, in which most but not all of the taxa in Table 9 are included. The vertical scale here does not correspond with depths in any sediment column, but is such that a constant width is assigned to each of the planktonic foraminiferal zones shown in the biostratigraphic summary chapter. In Figure 2, the limits of ranges are plotted according to their observed positions in a sequence of selected Leg 15 cores-Cores 151-4 and 151-5, $149-23$ to $149-30,153-7,149-31$ to $149-43$ and $152-1$ to 152-9. Arrows indicate that ranges extend for an unknown distance beyond the plotted lines. The ranges plotted are for morphotypes, and evolutionary limits are not shown.
TABLE 8

Radiolarians from Site 153 (Lower Portion)

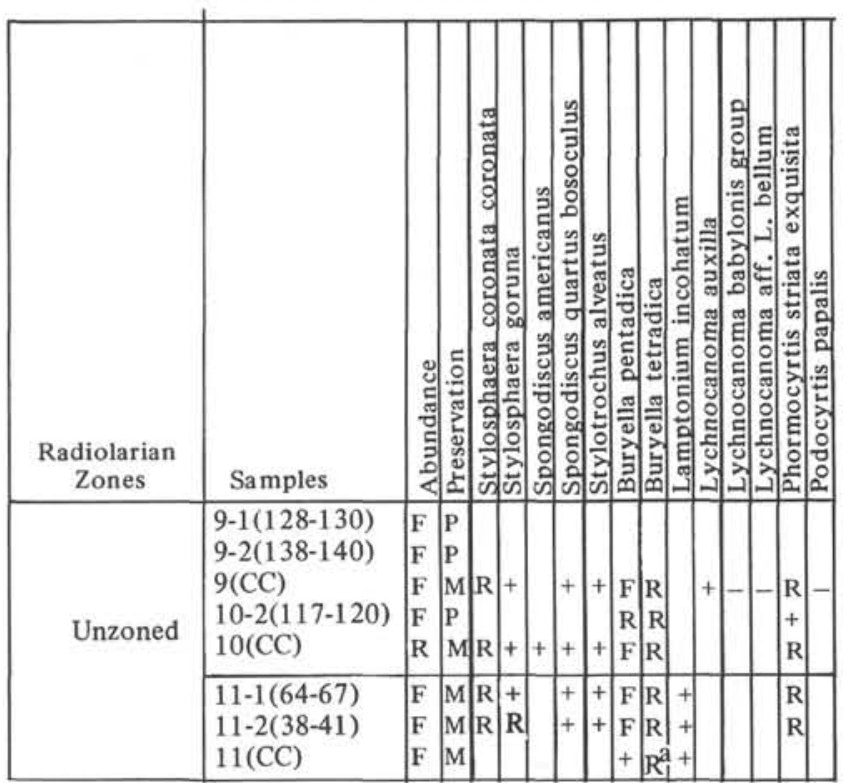

${ }^{\mathrm{a}}$ Specimens of Buryella tetradica in this assemblage commonly have the fourth (downwardly tapering) segment divided rather than simple, as in Plate 5, Figure 5.

Some ranges do not correspond completely with the order given in Table 9, since information from other legs as well was taken into account in ordering that list of events. 


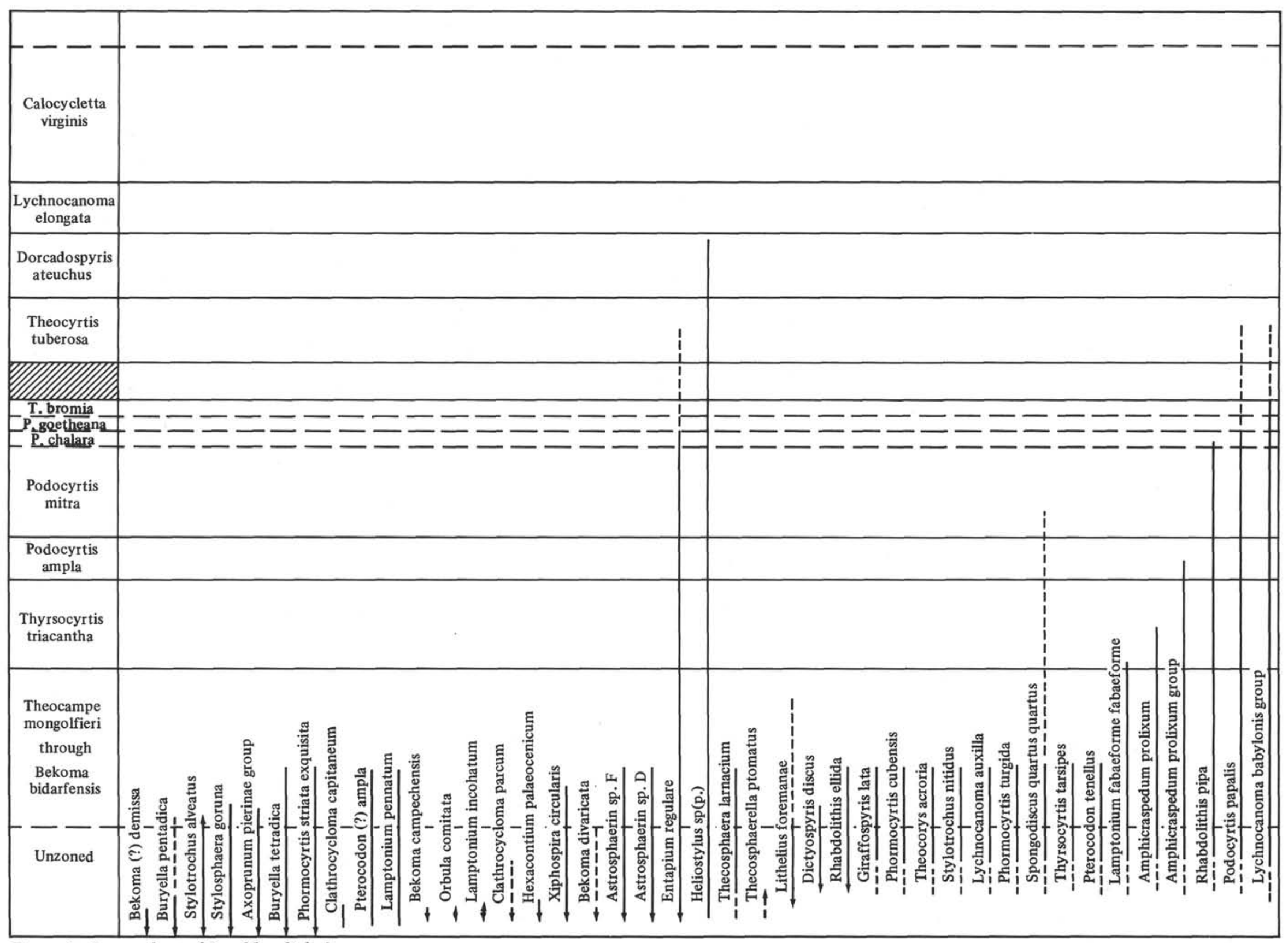




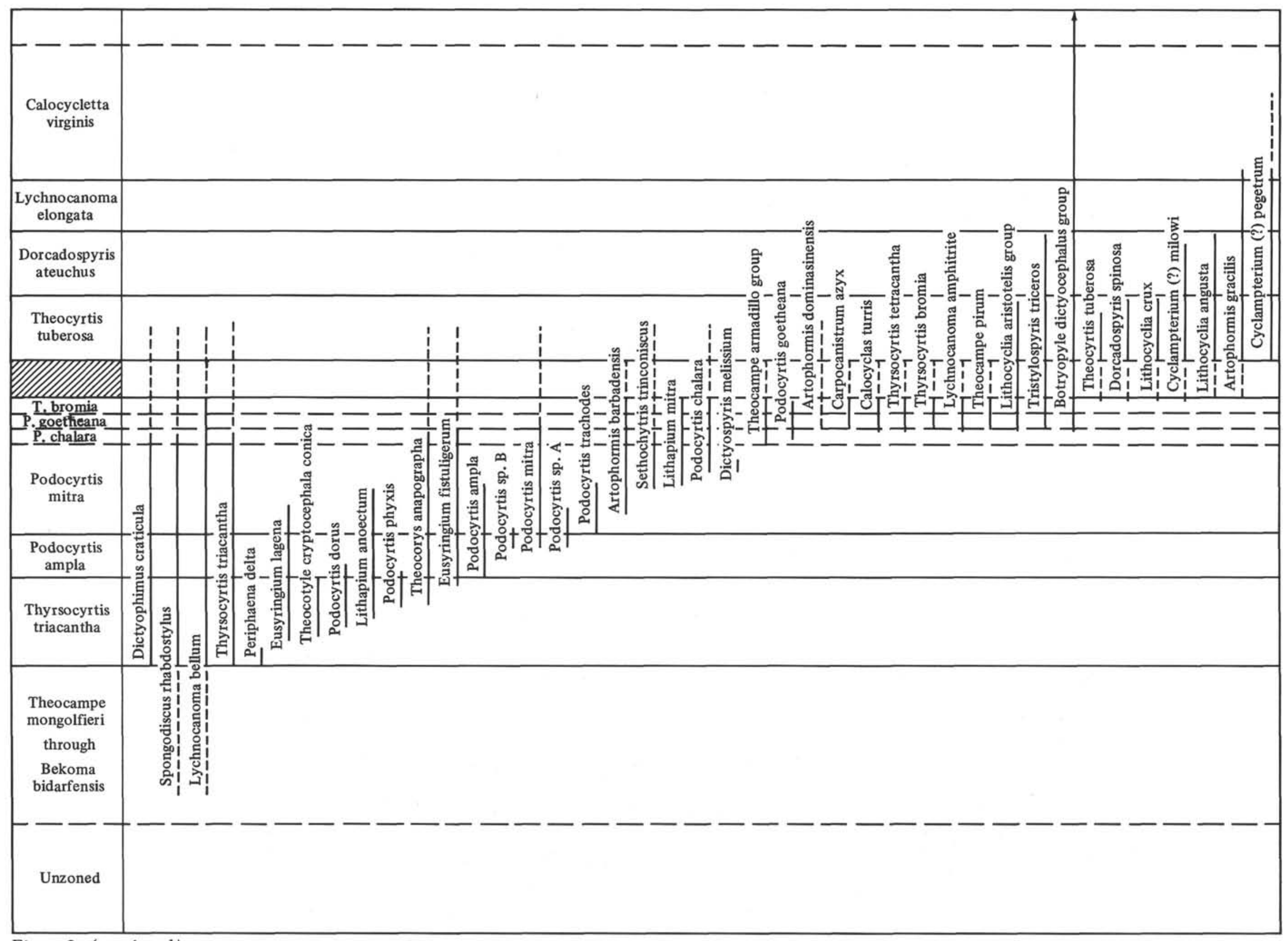




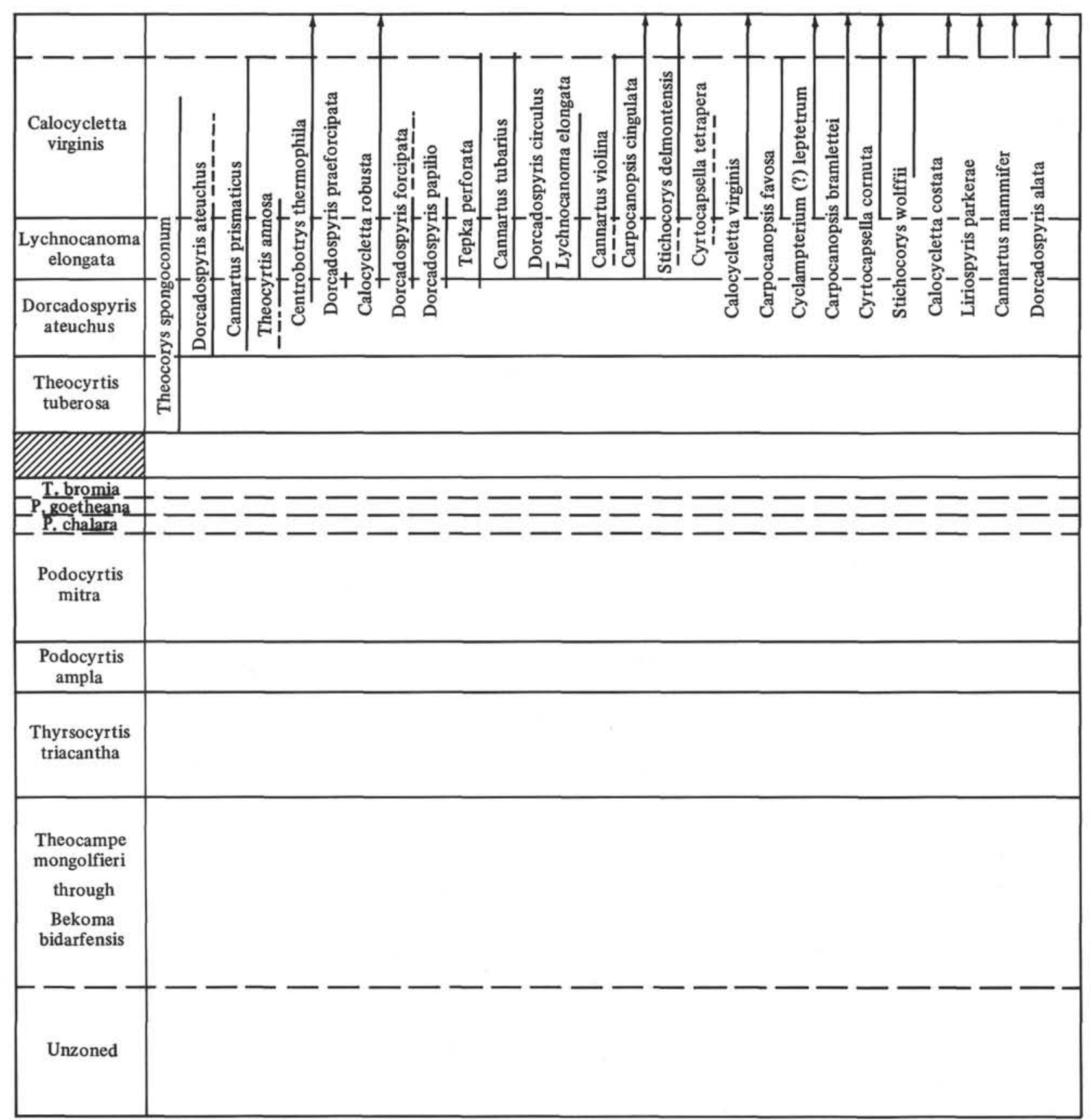


TABLE 9

Radiolarian Events at Sites 146, 149, 151, 152, and 153

(For Explanation, See Text)

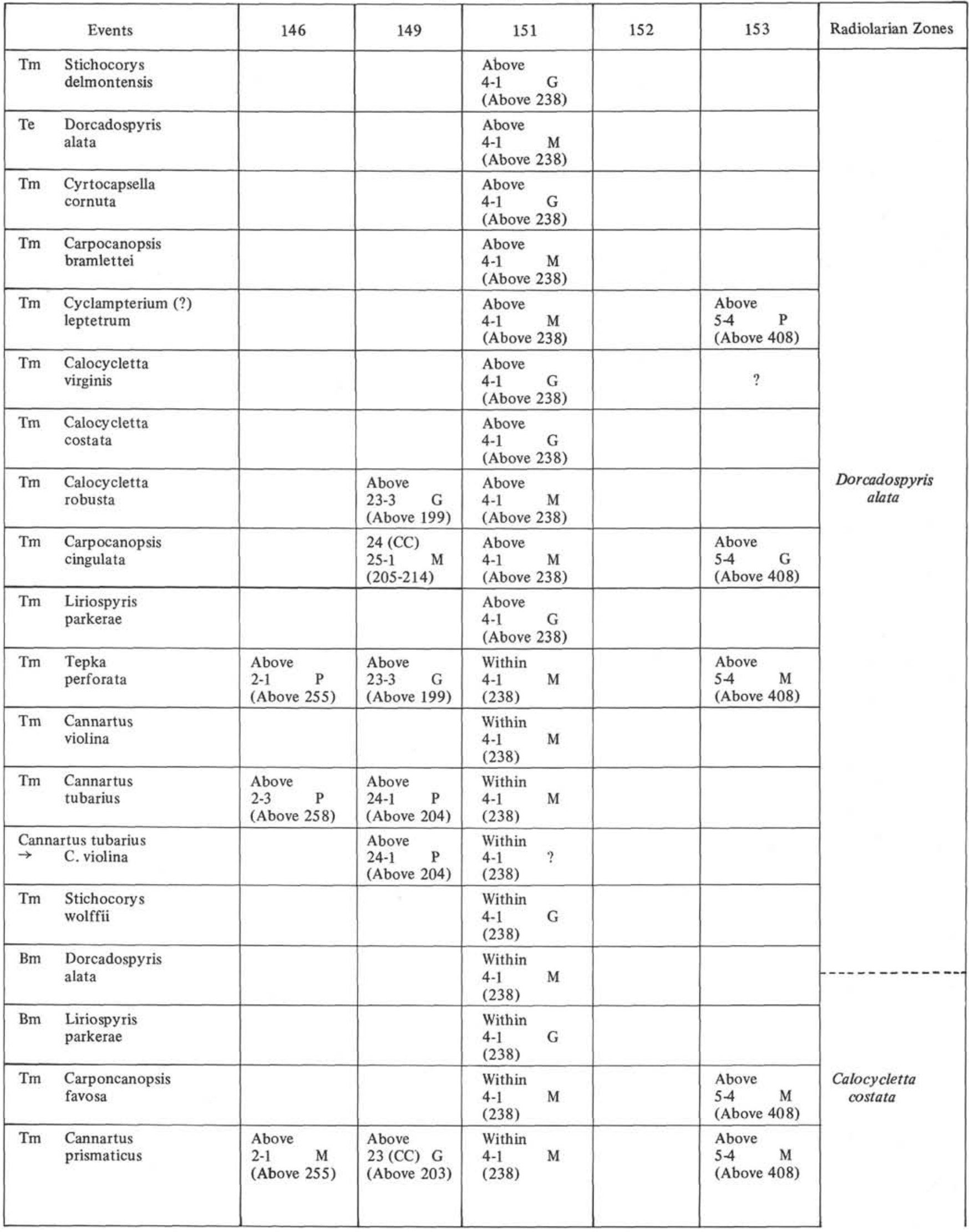


TABLE 9 - Continued

\begin{tabular}{|c|c|c|c|c|c|c|c|}
\hline & Events & 146 & 149 & 151 & 152 & 153 & Radiolarian Zones \\
\hline \multirow[t]{2}{*}{ Bm-e } & \multirow{2}{*}{$\begin{array}{l}\text { Calocycletta } \\
\text { costata }\end{array}$} & & & \multirow{2}{*}{$\begin{array}{l}\text { Within } \\
4-1 \\
(238)\end{array} \quad$ G } & & & \\
\hline & & & & & & & \multirow{17}{*}{$\begin{array}{c}\text { Calocycletta } \\
\text { virginis }\end{array}$} \\
\hline $\mathrm{Tm}$ & $\begin{array}{l}\text { Theocorys } \\
\text { spongoconum }\end{array}$ & $\begin{array}{l}\text { Above } \\
2-1 \quad P \\
\text { (Above } 255 \text { ) }\end{array}$ & $\begin{array}{l}\text { Above } \\
23-5 \quad M \\
\text { (Above 202) }\end{array}$ & $\begin{array}{l}4-1 \\
4-2 \quad P \\
(238-239)\end{array}$ & & $\begin{array}{l}\text { Above } \\
5-4 \quad M \\
\text { (Above } 408 \text { ) } \\
\end{array}$ & \\
\hline $\operatorname{Tm}$ & $\begin{array}{l}\text { Cyrtocapsella } \\
\text { tetrapera }\end{array}$ & & $\begin{array}{l}\text { Above } \\
23-5 \quad P \\
\text { (Above 202) }\end{array}$ & $\begin{array}{l}4-2 \\
4(\mathrm{CC}) \quad \text { G } \\
(239-240)\end{array}$ & & $\begin{array}{l}\text { Above } \\
5-4 \quad M \\
\text { (Above } 408 \text { ) }\end{array}$ & \\
\hline $\operatorname{Tm}$ & $\begin{array}{l}\text { Lychnocanoma } \\
\text { elongata }\end{array}$ & $\begin{array}{l}\text { Above } \\
2-1 \quad G \\
\text { (Above 255) }\end{array}$ & $\begin{array}{l}\text { Above } \\
23-5 \quad M \\
\text { (Above 202) }\end{array}$ & $\begin{array}{l}4(C C) \\
5-1 \quad G \\
(240-303)\end{array}$ & & $\begin{array}{l}\text { Above } \\
5-4 \quad M \\
\text { (Above } 408 \text { ) } \\
\end{array}$ & \\
\hline Bm-e & $\begin{array}{l}\text { Stichocorys } \\
\text { wolffii }\end{array}$ & & & $\begin{array}{l}4(C C) \\
5-2 \quad G \\
(240-304)\end{array}$ & & & \\
\hline $\mathrm{Tm}$ & $\begin{array}{l}\text { Dorcadospyris } \\
\text { ateuchus }\end{array}$ & 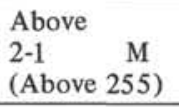 & $\begin{array}{l}\text { Above } \\
23(\mathrm{CC}) \mathrm{G} \\
\text { (Above 203) }\end{array}$ & $\begin{array}{l}\text { Within } \\
5-2 \\
(304)\end{array}$ & & $\begin{array}{l}\text { Above } \\
5-4 \quad M \\
\text { (Above } 408 \text { ) } \\
\end{array}$ & \\
\hline $\mathrm{Tm}$ & $\begin{array}{l}\text { Dorcadospyris } \\
\text { papilio }\end{array}$ & $\begin{array}{l}\text { Above } \\
2-1 \quad M \\
\text { (Above } 255 \text { ) }\end{array}$ & $\begin{array}{l}25-1 \\
25-2 \quad P \\
(214-215)\end{array}$ & $\begin{array}{l}\text { Within } \\
5-2 \\
(304)\end{array}$ & & & \\
\hline $\mathrm{Tm}$ & $\begin{array}{l}\text { Cyclampterium (?) } \\
\text { pegetrum }\end{array}$ & $\begin{array}{l}\text { Above } \\
2-1 \quad P \\
\text { (Above } 255 \text { ) }\end{array}$ & $\begin{array}{l}\text { Above } \\
23(\mathrm{CC}) \mathrm{M} \\
\text { (Above 203) }\end{array}$ & $\begin{array}{l}\text { Within } \\
5-2 \\
(304)\end{array}$ & & $\begin{array}{l}\text { Above } \\
5-4 \quad M \\
\text { (Above } 408 \text { ) }\end{array}$ & \\
\hline \multicolumn{2}{|c|}{$\begin{array}{l}\text { Cyclampterium (?) pegetrum } \\
\rightarrow \quad \text { C. (?) leptetrum }\end{array}$} & & $\begin{array}{l}\text { Above } \\
23(\mathrm{CC}) \mathrm{M} \\
\text { (Above 203) }\end{array}$ & $\begin{array}{l}\text { Within } \\
5-2 \\
(304)\end{array}$ & & & \\
\hline $\mathrm{Bm}$ & $\begin{array}{l}\text { Cyclampterium (?) } \\
\text { leptetrum }\end{array}$ & & & $\begin{array}{l}\text { Within } \\
5-2 \\
(304)\end{array}$ & & $?$ & \\
\hline $\operatorname{Tm}$ & $\begin{array}{l}\text { Theocyrtis } \\
\text { annosa }\end{array}$ & $\begin{array}{l}\text { Above } \\
2-1 \quad M \\
\text { (Above } 255 \text { ) }\end{array}$ & $\begin{array}{l}\text { Above } \\
23-5 \quad G \\
\text { (Above 202) }\end{array}$ & $\begin{array}{l}\text { Within } \\
5-2 \\
(304)\end{array} \quad$ G & & $\begin{array}{l}5(\mathrm{CC}) \quad \mathrm{M} \\
6-1 \quad \mathrm{M} \\
(410-413)\end{array}$ & \\
\hline $\operatorname{Tm}$ & $\begin{array}{l}\text { Buryella } \\
\text { clinata }\end{array}$ & $\begin{array}{l}\text { Above } \\
4 \text { (CC) M } \\
\text { (Above 413) }\end{array}$ & & & & & \\
\hline $\mathrm{Bm}$ & $\begin{array}{l}\text { Carpocanopsis } \\
\text { bramlettei }\end{array}$ & & & $\begin{array}{l}\text { Within } \\
5-2 \\
(304)\end{array}$ & & & \\
\hline $\mathrm{Bm}$ & $\begin{array}{l}\text { Cyrtocapsella } \\
\text { cornuta }\end{array}$ & & & $\begin{array}{l}\text { Within } \\
5-2 \\
(304)\end{array}$ & & & \\
\hline $\mathrm{Bm}$ & $\begin{array}{l}\text { Carpocanopsis } \\
\text { favosa }\end{array}$ & & & $\begin{array}{l}\text { Within } \\
5-2 \\
(304)\end{array}$ & & $\begin{array}{l}6-2 \\
6(\mathrm{CC}) \quad P \\
(414-415) \\
\end{array}$ & \\
\hline $\mathrm{Bm}$ & $\begin{array}{l}\text { Cyrtocapsella } \\
\text { tetrapera }\end{array}$ & & $\begin{array}{l}23-5 \\
23(\mathrm{CC}) \quad P \\
(202-203)\end{array}$ & $\begin{array}{l}\text { Within } \\
5-2 \\
(304)\end{array}$ & & $\begin{array}{l}6(\mathrm{CC}) \\
7-1 \quad \mathrm{M} \\
(415-500)\end{array}$ & \\
\hline \multirow[t]{2}{*}{$\mathrm{Bm}$} & \multirow{2}{*}{$\begin{array}{l}\text { Calocycletta } \\
\text { virginis }\end{array}$} & & & Within & & $6-1$ & \\
\hline & & & & $\begin{array}{ll}5-2 & \text { G } \\
(304) & \\
\end{array}$ & & $\begin{array}{l}7-1 \quad \text { G } \\
(413-500)\end{array}$ & \multirow{4}{*}{$\begin{array}{c}\text { Lychnocanoma } \\
\text { elongata }\end{array}$} \\
\hline $\mathrm{Tm}$ & $\begin{array}{l}\text { Artophormis } \\
\text { gracilis }\end{array}$ & $\begin{array}{l}\text { Above } \\
2-3 \quad P \\
\text { (Above } 258 \text { ) }\end{array}$ & $\begin{array}{l}\text { Within } \\
25-2 \\
(215)\end{array} \quad$ G & $\begin{array}{l}\text { Within } \\
5-2 \\
(304)\end{array}$ & & $\begin{array}{l}6(\mathrm{CC}) \\
7-1 \quad M \\
(415-500)\end{array}$ & \\
\hline $\mathrm{Bm}$ & $\begin{array}{l}\text { Stichocorys } \\
\text { delmontensis }\end{array}$ & & & $\begin{array}{l}5-2 \\
6-1 \quad G \\
(304-312)\end{array}$ & & 0 & \\
\hline $\operatorname{Tm}$ & $\begin{array}{l}\text { Dorcadospyris } \\
\text { circulus }\end{array}$ & & $\begin{array}{l}25(C C) \\
26-1 \quad M \\
(218-224)\end{array}$ & & & & \\
\hline
\end{tabular}


TABLE 9 - Continued

\begin{tabular}{|c|c|c|c|c|c|c|c|}
\hline & Events & 146 & 149 & 151 & 152 & 153 & Radiolarian Zones \\
\hline $\mathrm{Tm}$ & $\begin{array}{l}\text { Dorcadospyris } \\
\text { forcipata }\end{array}$ & $\begin{array}{l}\text { Above } \\
2-2 \\
\text { (Above } 256 \text { ) }\end{array}$ & $\begin{array}{l}25(\mathrm{CC}) \\
26-2 \quad \mathrm{M} \\
(218-225)\end{array}$ & $\begin{array}{l}\text { Within } \\
4-1 \quad \text { G } \\
(238)\end{array}$ & & & \multirow{5}{*}{$\begin{array}{l}\text { Lychnocanoma } \\
\text { elongata }\end{array}$} \\
\hline $\mathrm{Bm}$ & $\begin{array}{l}\text { Cannartus } \\
\text { violina }\end{array}$ & & & $\begin{array}{l}5-2 \\
6-1 \quad M \\
(304-312)\end{array}$ & & & \\
\hline $\mathrm{Tm}$ & $\begin{array}{l}\text { Dorcadospyris } \\
\text { praeforcipata }\end{array}$ & $\begin{array}{l}2-3 \\
2-4 \quad M \\
(258-259)\end{array}$ & $\begin{array}{l}\text { Within } \\
26-2\end{array}$ & $\begin{array}{lr}5-2 & \\
6-1 & P \\
(304-312)\end{array}$ & & & \\
\hline $\mathrm{Bm}$ & $\begin{array}{l}\text { Dorcadospyris } \\
\text { circulus }\end{array}$ & & $\begin{array}{l}26-3 \\
26(\mathrm{CC}) \quad \mathrm{M} \\
(226-227)\end{array}$ & & & & \\
\hline \multirow[t]{2}{*}{$\mathrm{Bm}$} & \multirow{2}{*}{$\begin{array}{l}\text { Lychnocanoma } \\
\text { elongata }\end{array}$} & \multirow{2}{*}{$\begin{array}{l}\text { Within } \\
2-4 \quad \text { M } \\
(259)\end{array}$} & \multirow{2}{*}{$\begin{array}{l}26-3 \\
26(\mathrm{CC}) \quad \mathrm{M} \\
(226-227)\end{array}$} & \multirow{2}{*}{$\begin{array}{l}5-2 \\
6-1 \quad G \\
(304-312)\end{array}$} & & \multirow{2}{*}{$\begin{array}{l}6(\mathrm{CC}) \\
7-1 \quad M \\
(415-500)\end{array}$} & \\
\hline & & & & & & & \multirow{14}{*}{$\begin{array}{l}\text { Dorcadospyris } \\
\text { ateuchus }\end{array}$} \\
\hline $\mathrm{Bm}$ & $\begin{array}{l}\text { Carpocanopsis } \\
\text { cingulata }\end{array}$ & & $\begin{array}{l}26(\mathrm{CC}) \\
27-2 \quad \mathrm{M} \\
(227-234)\end{array}$ & $\begin{array}{l}5-2 \\
6-1 \quad M \\
(304-312)\end{array}$ & & $\begin{array}{l}\text { Below } \\
6 \text { (CC) M } \\
\text { (Below 415) }\end{array}$ & \\
\hline $\mathrm{Bm}$ & $\begin{array}{l}\text { Tepka } \\
\text { perforata }\end{array}$ & $\begin{array}{l}\text { Within } \\
2-4 \\
(259)\end{array}$ & $\begin{array}{l}26(\mathrm{CC}) \\
27-2 \quad M \\
(227-234)\end{array}$ & $\begin{array}{l}5-2 \\
6-1 \quad P \\
(304-312)\end{array}$ & & $\begin{array}{l}\text { Below } \\
6 \text { (CC) P } \\
\text { (Below 415) }\end{array}$ & \\
\hline $\operatorname{Tm}$ & $\begin{array}{l}\text { Lithocyclia } \\
\text { angusta }\end{array}$ & $\begin{array}{l}2-5 \\
2-6 \quad M \\
(261-262)\end{array}$ & $\begin{array}{l}26(\mathrm{CC}) \\
27-2 \quad M \\
(227-234)\end{array}$ & $\begin{array}{l}6-2 \\
6-3 \quad M \\
(313-314)\end{array}$ & & $\begin{array}{l}\begin{array}{l}6(\mathrm{CC}) \\
7-1 \quad M \\
(415-500)\end{array} \\
\end{array}$ & \\
\hline \multicolumn{2}{|c|}{$\begin{array}{l}\text { Lithocyclia angusta } \\
\rightarrow \quad \text { Cannartus prismaticus }\end{array}$} & $\begin{array}{l}2-6 \\
2(\mathrm{CC}) \quad M \\
(262-263)\end{array}$ & $\begin{array}{l}26(C C) \\
27-2 \quad G \\
(227-234)\end{array}$ & $\begin{array}{l}6-3 \\
8(\mathrm{CC}) \quad \mathrm{M} \\
(314-330)\end{array}$ & & $\begin{array}{l}6(\mathrm{CC}) \\
7-1 \quad M \\
(415-500)\end{array}$ & \\
\hline $\mathrm{Bm}$ & $\begin{array}{l}\text { Dorcadospyris } \\
\text { forcipata }\end{array}$ & $\begin{array}{l}\text { Below } \\
2 \text { (CC) M } \\
\text { (Below 263) }\end{array}$ & $\begin{array}{l}27-2 \\
27-3 \quad M \\
(234-236)\end{array}$ & $\begin{array}{l}7-1 \\
7-2 \quad M \\
(321-322)\end{array}$ & & & \\
\hline $\mathrm{Bm}$ & $\begin{array}{l}\text { Cannartus } \\
\text { tubarius }\end{array}$ & $\begin{array}{l}\text { Below } \\
2-4 \quad P \\
\text { (Below }\end{array}$ & $\begin{array}{l}26(\mathrm{CC}) \\
27-2 \quad P \\
(227-234)\end{array}$ & $\begin{array}{l}\text { Below } \\
8(\mathrm{CC}) \quad \mathrm{P} \\
\text { (Below } 330)\end{array}$ & & & \\
\hline $\mathrm{Bm}$ & $\begin{array}{l}\text { Dorcadospyris } \\
\text { papilio }\end{array}$ & $\begin{array}{l}2-6 \\
2(\mathrm{CC}) \quad P \\
(262-263)\end{array}$ & $\begin{array}{l}27-3 \\
27(\mathrm{CC}) \quad P \\
(236-237)\end{array}$ & $\begin{array}{l}\text { Below } \\
8-1 \quad M \\
\text { (Below } \\
\end{array}$ & & & \\
\hline $\operatorname{Tm}$ & $\begin{array}{l}\text { Tristylospyris } \\
\text { triceros }\end{array}$ & $\begin{array}{l}\text { Below } \\
2 \text { (CC) } \\
\text { (Below 263) }\end{array}$ & $\begin{array}{l}27-3 \\
27(C C) \quad P \\
(236-237)\end{array}$ & & & $\begin{array}{l}6(\mathrm{CC}) \\
7-1 \quad M \\
(415-500)\end{array}$ & \\
\hline $\mathrm{Bm}$ & $\begin{array}{l}\text { Calocycletta } \\
\text { robusta }\end{array}$ & & $\begin{array}{l}27-3 \\
27(C C) \quad G \\
(236-237)\end{array}$ & $\begin{array}{l}\text { Below } \\
8(\text { CC) M } \\
\text { (Below 330) }\end{array}$ & & & \\
\hline $\mathrm{Bm}$ & $\begin{array}{l}\text { Dorcadospyris } \\
\text { praeforcipata }\end{array}$ & $\begin{array}{l}\text { Below } \\
2 \text { (CC) M } \\
\text { (Below 263) } \\
\end{array}$ & $\begin{array}{l}27(\mathrm{CC}) \\
28-1 \quad P \\
(237-242)\end{array}$ & $\begin{array}{l}7-1 \\
7-2 \quad P \\
(321-322)\end{array}$ & & & \\
\hline $\mathrm{Tm}$ & Heliostylus sp (p) & $\begin{array}{l}\text { Above } \\
7-1 \quad P \\
\text { (Above } 441 \text { ) }\end{array}$ & $\begin{array}{l}27(\mathrm{CC}) \\
28-1 \quad P \\
(237-242)\end{array}$ & & $\begin{array}{l}\text { Above } \\
1-2 \quad G \\
\text { (Above } 155 \text { ) } \\
\end{array}$ & & \\
\hline $\mathrm{Tm}$ & $\begin{array}{l}\text { Cyclampterium (?) } \\
\text { milowi }\end{array}$ & & $\begin{array}{l}28-2 \\
28-3 \\
(243-244)\end{array}$ & $\begin{array}{l}8-1 \\
8(\mathrm{CC}) \quad P \\
(329-330)\end{array}$ & & $\begin{array}{l}6(C C) \\
7-1 \quad M \\
(415-500)\end{array}$ & \\
\hline $\mathrm{Bm}$ & $\begin{array}{l}\text { Centrobotrys } \\
\text { thermophila }\end{array}$ & & $\begin{array}{l}28-4 \\
28(\mathrm{CC}) \quad P \\
(245-246)\end{array}$ & & & & \\
\hline \multicolumn{2}{|c|}{$\begin{array}{l}\text { Tristylospyris triceros } \\
\rightarrow \quad \text { Dorcadospyris ateuchus }\end{array}$} & & $\begin{array}{l}28-4 \\
29-2 \\
(245-253)\end{array}$ & & & $\begin{array}{l}6(C C) \\
7-1 \quad M \\
(415-500)\end{array}$ & \multirow[b]{2}{*}{$\begin{array}{l}\text { Theocyrtis } \\
\text { tuberosa }\end{array}$} \\
\hline $\mathrm{Bm}$ & $\begin{array}{l}\text { Theocyrtis } \\
\text { annosa }\end{array}$ & $\begin{array}{l}\text { Below } \\
2 \text { (CC) P } \\
\text { (Below 263) }\end{array}$ & $\begin{array}{l}28(C C) \\
29-1 \quad M \\
(246-252)\end{array}$ & $\begin{array}{l}\text { Below } \\
8(C C) \quad \text { G } \\
\text { (Below } 330)\end{array}$ & & $\begin{array}{l}6(C C) \\
7-1 \quad M \\
(415-500)\end{array}$ & \\
\hline
\end{tabular}


TABLE 9 - Continued

\begin{tabular}{|c|c|c|c|c|c|c|c|}
\hline & Events & 146 & 149 & 151 & 152 & 153 & Radiolarian Zones \\
\hline $\mathrm{Bm}$ & $\begin{array}{l}\text { Cannartus } \\
\text { prismaticus }\end{array}$ & $\begin{array}{l}\text { Below } \\
2 \text { (CC) M } \\
\text { (Below 263) }\end{array}$ & 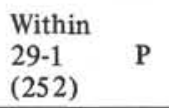 & $\begin{array}{l}\text { Below } \\
8(C C) \quad M \\
\text { (Below } 330)\end{array}$ & & $\begin{array}{l}\text { Below } \\
6 \text { (CC) M } \\
\text { (Below 415) }\end{array}$ & \multirow{21}{*}{$\begin{array}{c}\text { Theocyrtis } \\
\text { tuberosa }\end{array}$} \\
\hline $\mathrm{Tm}$ & $\begin{array}{l}\text { Lithomitra } \\
\text { docilis }\end{array}$ & & $\begin{array}{l}\text { Within } \\
29-2 \\
(253)\end{array} \quad$ M & & $\begin{array}{l}\text { Above } \\
3-5 \\
\text { (Above 177) }\end{array}$ & & \\
\hline $\mathrm{Bm}$ & $\begin{array}{l}\text { Dorcadospyris } \\
\text { ateuchus }\end{array}$ & $\begin{array}{l}\text { Below } \\
2(C C) \quad M \\
\text { (Below 263) }\end{array}$ & $\begin{array}{l}29-2 \\
29-3 \quad M \\
(253-254)\end{array}$ & $\begin{array}{l}\text { Below } \\
8(\text { CC) M } \\
\text { (Below 330) }\end{array}$ & & $\begin{array}{l}6(C C) \\
7-1 \quad M \\
(415-500)\end{array}$ & \\
\hline$\underset{\rightarrow}{\text { Cycl }}$ & $\begin{array}{l}\text { mpterium (?) milowi } \\
\text { (?) pegetrum }\end{array}$ & & $\begin{array}{l}28-3 \\
30-1 \quad M \\
(244-260)\end{array}$ & $\begin{array}{l}\text { Below } \\
8(C C) \quad M \\
\text { (Below } 330)\end{array}$ & & $\begin{array}{l}6(\mathrm{CC}) \\
7-1 \quad M \\
(415-500)\end{array}$ & \\
\hline $\mathrm{Tm}$ & $\begin{array}{l}\text { Dorcadospyris } \\
\text { spinosa }\end{array}$ & & $\begin{array}{l}29(\mathrm{CC}) \\
30-1 \quad \mathrm{M} \\
(255-260)\end{array}$ & & & 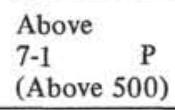 & \\
\hline $\mathrm{Tm}$ & $\begin{array}{l}\text { Lithocyclia } \\
\text { crux }\end{array}$ & & $\begin{array}{l}29(\mathrm{CC}) \\
30-1 \quad M \\
(255-260)\end{array}$ & & & $\begin{array}{l}6(\mathrm{CC}) \\
7-1 \quad P \\
(415-500)\end{array}$ & \\
\hline $\mathrm{Bm}$ & $\begin{array}{l}\text { Theocorys } \\
\text { spongoconum }\end{array}$ & $\begin{array}{l}\text { Below } \\
2(C C) \quad M \\
\text { (Below 263) }\end{array}$ & $\begin{array}{l}29(\mathrm{CC}) \\
30-1 \quad P \\
(255-260)\end{array}$ & $\begin{array}{l}\text { Below } \\
8(C C) \quad M \\
\text { (Below 330) }\end{array}$ & & $\begin{array}{l}\text { Below } \\
7 \text { (CC) M } \\
\text { (Below 501) }\end{array}$ & \\
\hline $\mathrm{Tm}$ & $\begin{array}{l}\text { Lithocyclia } \\
\text { aristotelis group }\end{array}$ & & $\begin{array}{l}\text { Within } \\
30-1 \quad \text { M } \\
(260)\end{array}$ & & & & \\
\hline $\mathrm{Tm}$ & $\begin{array}{l}\text { Theocyrtis } \\
\text { tuberosa }\end{array}$ & & $\begin{array}{l}\text { Within } \\
30-2 \\
(261)\end{array}$ & & & $\begin{array}{l}6(\mathrm{CC}) \\
7-1 \quad M \\
(415-500)\end{array}$ & \\
\hline $\mathrm{Bm}$ & $\begin{array}{l}\text { Cyclampterium (?) } \\
\text { pegetrum }\end{array}$ & $\begin{array}{l}\text { Below } \\
2(\mathrm{CC}) \quad \mathrm{P} \\
\text { (Below 263) }\end{array}$ & $\begin{array}{l}30-1 \\
30(\mathrm{CC}) \quad P \\
(260-262)\end{array}$ & $\begin{array}{l}\text { Below } \\
8(\mathrm{CC}) \quad \mathrm{M} \\
\text { (Below } 330 \text { ) }\end{array}$ & & $\begin{array}{l}\text { Below } \\
7(\mathrm{CC}) \quad \mathrm{P} \\
\text { (Below 501) }\end{array}$ & \\
\hline $\mathrm{Bm}$ & $\begin{array}{l}\text { Centrobotrys } \\
\text { petrushevskayae }\end{array}$ & & $\begin{array}{l}30-2 \\
30(\mathrm{CC}) \quad \mathrm{G} \\
(261-262)\end{array}$ & $?$ & & & \\
\hline $\mathrm{Bm}$ & $\begin{array}{l}\text { Lithocyclia } \\
\text { crux }\end{array}$ & & $\begin{array}{l}30(\mathrm{CC}) \\
31-1 \quad \mathrm{M} \\
(262-271\end{array}$ & & & $\begin{array}{l}\text { Below } \\
7(\mathrm{CC}) \quad \mathrm{P} \\
\text { (Below 501) }\end{array}$ & \\
\hline \multirow{2}{*}{\multicolumn{2}{|c|}{$\begin{array}{l}\text { Lithocyclia aristotelis group } \\
\rightarrow \quad \text { L. angusta }\end{array}$}} & & \multirow{2}{*}{$\begin{array}{l}30-1 \\
31-1 \quad G \\
(260-271)\end{array}$} & & & & \\
\hline & & & & & & & \\
\hline $\operatorname{Tm}$ & $\begin{array}{l}\text { Artophormis } \\
\text { barbadensis }\end{array}$ & & $\begin{array}{l}30(\mathrm{CC}) \\
31-1 \quad \mathrm{P} \\
(262-271)\end{array}$ & & & $\begin{array}{l}\text { Below } \\
7 \text { (CC) M } \\
\text { (Below 501) } \\
\end{array}$ & \\
\hline \multicolumn{2}{|c|}{$\begin{array}{l}\text { Artophormis barbadensis } \\
\rightarrow \quad \text { A. gracilis }\end{array}$} & & $\begin{array}{l}30(C C) \\
31-1 \\
(262-271)\end{array}$ & & & & \\
\hline $\mathrm{Bm}$ & $\begin{array}{l}\text { Dorcadospyris } \\
\text { spinosa }\end{array}$ & & $\begin{array}{l}30(\mathrm{CC}) \\
31-1 \quad \mathrm{M} \\
(262-271)\end{array}$ & & & $\begin{array}{l}\text { Below } \\
7(\text { CC) P } \\
\text { (Below 501) }\end{array}$ & \\
\hline $\mathrm{Tm}$ & $\begin{array}{l}\text { Theocampe } \\
\text { amphora group }\end{array}$ & & $\begin{array}{l}30(\mathrm{CC}) \\
32-2 \quad \mathrm{P} \\
(262-281)\end{array}$ & & & & \\
\hline $\mathrm{Tm}$ & $\begin{array}{l}\text { Theocampe } \\
\text { pirum }\end{array}$ & & $\begin{array}{l}30(C C) \\
31-1 \quad M \\
(262-271)\end{array}$ & & & $\begin{array}{lr}\text { Below } & \\
7 \text { (CC) } & \text { M } \\
\text { (Below 501) }\end{array}$ & \\
\hline $\mathrm{Tm}$ & $\begin{array}{l}\text { Lychnocanoma } \\
\text { babylonis group }\end{array}$ & $\begin{array}{l}\text { Above } \\
7-1 \quad M \\
\text { (Above } 441 \text { ) }\end{array}$ & $\begin{array}{l}30(\mathrm{CC}) \\
31-1 \quad P \\
(262-271)\end{array}$ & & $\begin{array}{l}\text { Above } \\
2-5 \\
\text { (Above 167) }\end{array}$ & & \\
\hline $\mathrm{Tm}$ & $\begin{array}{l}\text { Theocampe } \\
\text { mongolfieri }\end{array}$ & & $\begin{array}{l}30(\mathrm{CC}) \\
31-1 \\
(262-271)\end{array}$ & & & $\begin{array}{l}6(C C) \\
7-1 \\
(415-500)\end{array}$ & \\
\hline
\end{tabular}


TABLE 9 - Continued

\begin{tabular}{|c|c|c|c|c|c|c|c|}
\hline & Events & 146 & 149 & 151 & 152 & 153 & Radiolarian Zones \\
\hline $\mathrm{Tm}$ & $\begin{array}{l}\text { Lophocyrtis } \\
\text { jacchia }\end{array}$ & & $\begin{array}{l}30(\mathrm{CC}) \\
31-1 \quad \mathrm{M} \\
(262-271)\end{array}$ & & & & \multirow{20}{*}{$\begin{array}{l}\text { Thyrsocyrtis } \\
\text { bromia }\end{array}$} \\
\hline $\mathrm{Tm}$ & $\begin{array}{l}\text { Lychnocanoma } \\
\text { amphitrite }\end{array}$ & & $\begin{array}{l}30(\mathrm{CC}) \\
31-1 \quad \mathrm{M} \\
(262-271)\end{array}$ & & & $\begin{array}{l}\text { Below } \\
7(\text { CC) M } \\
\text { (Below 501) }\end{array}$ & \\
\hline $\mathrm{Tm}$ & $\begin{array}{l}\text { Theocampe } \\
\text { armadillo group }\end{array}$ & & $\begin{array}{l}30(\mathrm{CC}) \\
31-1 \quad \mathrm{M} \\
(262-271)\end{array}$ & & & $\begin{array}{l}\text { Below } \\
7(\mathrm{CC}) \quad \mathrm{M} \\
\text { (Below 501) } \\
\end{array}$ & \\
\hline $\mathrm{Tm}$ & $\begin{array}{l}\text { Periphaena } \\
\text { decora }\end{array}$ & & $\begin{array}{l}30(\mathrm{CC}) \\
31-1 \quad \mathrm{M} \\
(262-271)\end{array}$ & . & & $\begin{array}{l}5(C C) \\
6-1 \quad P \\
(410-413)\end{array}$ & \\
\hline $\mathrm{Bm}$ & $\begin{array}{l}\text { Artophormis } \\
\text { gracilis }\end{array}$ & $\begin{array}{l}\text { Below } \\
2(\text { CC) M } \\
\text { (Below 263) }\end{array}$ & $\begin{array}{l}30(\mathrm{CC}) \\
31-1 \quad \mathrm{G} \\
(262-271)\end{array}$ & $\begin{array}{lr}\text { Below } & \\
8(\text { CC) } & \text { M } \\
\text { (Below } 330)\end{array}$ & & $\begin{array}{l}\text { Below } \\
7(\mathrm{CC}) \quad \mathrm{M} \\
\text { (Below 501) }\end{array}$ & \\
\hline $\mathrm{Bm}$ & $\begin{array}{l}\text { Cyclampterium (?) } \\
\text { milowi }\end{array}$ & & $\begin{array}{l}30(\mathrm{CC}) \\
31-1 \quad \mathrm{M} \\
(262-271)\end{array}$ & $\begin{array}{l}\text { Below } \\
8(\text { CC) } \quad \text { P } \\
\text { (Below 330) }\end{array}$ & & $\begin{array}{l}\text { Below } \\
7 \text { (CC) M } \\
\text { (Below 501) }\end{array}$ & \\
\hline $\mathrm{Tm}$ & $\begin{array}{l}\text { Theocampe } \\
\text { urceolus }\end{array}$ & & $\begin{array}{l}30(\mathrm{CC}) \\
32-2 \quad P \\
(262-281)\end{array}$ & & & & \\
\hline $\mathrm{Bm}$ & $\begin{array}{l}\text { Theocyrtis } \\
\text { tuberosa }\end{array}$ & & $\begin{array}{l}30(\mathrm{CC}) \\
31-1 \quad \mathrm{G} \\
(262-271)\end{array}$ & & & $\begin{array}{l}\text { Below } \\
7(\mathrm{CC}) \quad \mathrm{M} \\
\text { (Below 501) }\end{array}$ & \\
\hline $\mathrm{Bm}$ & $\begin{array}{l}\text { Lithocy clia } \\
\text { angusta }\end{array}$ & $\begin{array}{l}\text { Below } \\
2(\text { CC) G } \\
\text { (Below 263) }\end{array}$ & $\begin{array}{l}30(\mathrm{CC}) \\
31-1 \quad \mathrm{M} \\
(262-271)\end{array}$ & $\begin{array}{l}\text { Below } \\
8(\text { CC) } \\
\text { (Below 330) }\end{array}$ & & $\begin{array}{l}\text { Below } \\
7 \text { (CC) M } \\
\text { (Below 501) }\end{array}$ & \\
\hline $\mathrm{Tm}$ & $\begin{array}{l}\text { Thyrsocyrtis } \\
\text { bromia }\end{array}$ & & $\begin{array}{l}30(\mathrm{CC}) \\
31-1 \quad \mathrm{G} \\
(262-271)\end{array}$ & & & $\begin{array}{l}\text { Below } \\
7 \text { (CC) M } \\
\text { (Below 501) }\end{array}$ & \\
\hline $\mathrm{Tm}$ & $\begin{array}{l}\text { Thyrsocyrtis } \\
\text { tetracantha }\end{array}$ & & $\begin{array}{l}30(\mathrm{CC}) \\
31-1 \quad \mathrm{M} \\
(262-271)\end{array}$ & & & $\begin{array}{l}\text { Below } \\
7 \text { (CC) M } \\
\text { (Below 501) }\end{array}$ & \\
\hline $\mathrm{Tm}$ & $\begin{array}{l}\text { Calocyclas } \\
\text { turris }\end{array}$ & & $\begin{array}{l}30(\mathrm{CC}) \\
31-1 \quad \mathrm{G} \\
(262-271)\end{array}$ & & & $\begin{array}{l}\text { Below } \\
7(\mathrm{CC}) \quad \mathrm{P} \\
\text { (Below 501) }\end{array}$ & \\
\hline $\mathrm{Tm}$ & $\begin{array}{l}\text { Thyrsocyrtis } \\
\text { rhizodon }\end{array}$ & & $\begin{array}{l}30(\mathrm{CC}) \\
31-1 \quad \mathrm{M} \\
(262-271)\end{array}$ & & & & \\
\hline $\mathrm{Tm}$ & $\begin{array}{l}\text { Thyrsocyrtis } \\
\text { triacantha }\end{array}$ & & $\begin{array}{l}30-2 \\
32-2 \quad P \\
(261-281)\end{array}$ & & & & \\
\hline $\mathrm{Tm}$ & $\begin{array}{l}\text { Lychnocanoma } \\
\text { bellum }\end{array}$ & & $\begin{array}{l}30(\mathrm{CC}) \\
31-1 \quad P \\
(262-271)\end{array}$ & & & & \\
\hline $\mathrm{Tm}$ & $\begin{array}{l}\text { Podocyrtis } \\
\text { chalara }\end{array}$ & & $\begin{array}{l}30(\mathrm{CC}) \\
31(\mathrm{CC}) \quad \mathrm{P} \\
(262-273)\end{array}$ & & & & \\
\hline $\mathrm{Tm}$ & $\begin{array}{l}\text { Eusyringium } \\
\text { fistuligerum }\end{array}$ & & $\begin{array}{l}30(\mathrm{CC}) \\
32-2 \quad P \\
(262-281)\end{array}$ & & & & \\
\hline $\mathrm{Tm}$ & $\begin{array}{l}\text { Calocyclas } \\
\text { hispida }\end{array}$ & & $\begin{array}{l}30(\mathrm{CC}) \\
32-2 \quad \mathrm{P} \\
(262-281)\end{array}$ & & & & \\
\hline $\mathrm{Bm}$ & $\begin{array}{l}\text { Lophocyrtis } \\
\text { jacchia }\end{array}$ & & $\begin{array}{l}31(\mathrm{CC}) \\
21-1 \quad P \\
(273-280)\end{array}$ & & & & \\
\hline $\mathrm{Tm}$ & $\begin{array}{l}\text { Podocyrtis } \\
\text { papalis }\end{array}$ & $\begin{array}{l}\text { Above } \\
4 \text { (CC) P } \\
\text { (Above 413) }\end{array}$ & $\begin{array}{l}30(\mathrm{CC}) \\
32-3 \quad P \\
(262-282)\end{array}$ & & $\begin{array}{l}\text { Above } \\
1-1 \quad \text { G } \\
\text { (Above 154) }\end{array}$ & & \\
\hline
\end{tabular}


TABLE 9 - Continued

\begin{tabular}{|c|c|c|c|c|c|c|c|}
\hline & Events & 146 & 149 & 151 & 152 & 153 & Radiolarian Zones \\
\hline $\mathrm{Tm}$ & $\begin{array}{l}\text { Lithocyclia } \\
\text { ocellus group }\end{array}$ & & $\begin{array}{l}30(\mathrm{CC}) \\
32-3 \quad P \\
(262-282)\end{array}$ & & & & \multirow{20}{*}{$\begin{array}{l}\text { Thyrsocyrtis } \\
\text { bromia }\end{array}$} \\
\hline $\operatorname{Tm}$ & $\begin{array}{l}\text { Sethochytris } \\
\text { triconiscus }\end{array}$ & & $\begin{array}{l}30(\mathrm{CC}) \\
32-4 \quad P \\
(262-283)\end{array}$ & & & & \\
\hline $\mathrm{Tm}$ & $\begin{array}{l}\text { Carpocanistrum } \\
\text { azyx }\end{array}$ & & $\begin{array}{l}30(\mathrm{CC}) \\
31-1 \quad \mathrm{M} \\
(262-271)\end{array}$ & & & $\begin{array}{l}\text { Below } \\
7 \text { (CC) M } \\
\text { (Below 501) }\end{array}$ & \\
\hline $\operatorname{Tm}$ & $\begin{array}{l}\text { Spongodiscus } \\
\text { rhabdostylus }\end{array}$ & & $\begin{array}{l}30(\mathrm{CC}) \\
32-4 \quad \mathrm{P} \\
(262-283)\end{array}$ & & & & \\
\hline $\mathrm{Tm}$ & $\begin{array}{l}\text { Entapium } \\
\text { regulare }\end{array}$ & $\begin{array}{l}\text { Above } \\
7-1 \quad \text { P } \\
\text { (Above } 441 \text { ) }\end{array}$ & $\begin{array}{l}30(\mathrm{CC}) \\
32-2 \quad P \\
(262-281)\end{array}$ & & $\begin{array}{l}\text { Above } \\
1-2 \quad G \\
\text { (Above } 155 \text { ) }\end{array}$ & & \\
\hline $\mathrm{Tm}$ & $\begin{array}{l}\text { Dictyophimus } \\
\text { craticula }\end{array}$ & & $\begin{array}{l}30(\mathrm{CC}) \\
32-4 \quad P \\
(262-283)\end{array}$ & & & & \\
\hline $\mathrm{Tm}$ & $\begin{array}{l}\text { Rhopalocanium } \\
\text { ornatum }\end{array}$ & & $\begin{array}{l}30(\mathrm{CC}) \\
32-4 \quad \mathrm{P} \\
(262-283)\end{array}$ & & & & \\
\hline $\mathrm{Tm}$ & $\begin{array}{l}\text { Spongatractus } \\
\text { pachystylus }\end{array}$ & & $\begin{array}{l}30(\mathrm{CC}) \\
32-2 \quad \mathrm{M} \\
(262-281)\end{array}$ & & & & \\
\hline $\mathrm{Tm}$ & $\begin{array}{l}\text { Theocorys } \\
\text { anapographa }\end{array}$ & & $\begin{array}{l}30(\mathrm{CC}) \\
32-4 \quad \mathrm{P} \\
(262-283)\end{array}$ & & & & \\
\hline $\mathrm{Te}$ & $\begin{array}{l}\text { Lithapium } \\
\text { mitra }\end{array}$ & & $\begin{array}{l}31-1 \\
31-2 \quad M \\
(271-272)\end{array}$ & & & & \\
\hline $\mathrm{Te}$ & $\begin{array}{l}\text { Podocyrtis } \\
\text { goetheana }\end{array}$ & & $\begin{array}{l}\text { Within } \\
31-2\end{array}$ & & & & \\
\hline $\mathrm{Tm}$ & $\begin{array}{l}\text { Artophormis } \\
\text { dominasinensis }\end{array}$ & & $\begin{array}{l}30(\mathrm{CC}) \\
32-2 \quad P \\
(262-281)\end{array}$ & & & & \\
\hline $\mathrm{Tm}$ & $\begin{array}{l}\text { Podocyrtis } \\
\text { mitra }\end{array}$ & & $\begin{array}{l}30(\mathrm{CC}) \\
32-2 \quad \mathrm{P} \\
(262-281)\end{array}$ & & & & \\
\hline $\mathrm{Bm}$ & $\begin{array}{l}\text { Botryopyle } \\
\text { dictyocephalus group }\end{array}$ & & $\begin{array}{l}32-3 \\
32-4 \quad M \\
(282-283)\end{array}$ & ? & & & \\
\hline $\mathrm{Bm}$ & $\begin{array}{l}\text { Thyrsocyrtis } \\
\text { tetracantha }\end{array}$ & & $\begin{array}{l}\text { Within } \\
32-4 \\
(283)\end{array}$ & & & & \\
\hline $\mathrm{Bm}$ & $\begin{array}{l}\text { Theocampe } \\
\text { pirum }\end{array}$ & & $\begin{array}{l}\text { Within } \\
32-4 \\
(283)\end{array}$ & & & & \\
\hline $\mathrm{Bm}$ & $\begin{array}{l}\text { Lychnocanoma } \\
\text { amphitrite }\end{array}$ & & $\begin{array}{l}\text { Within } \\
32-4 \\
(283)\end{array}$ & & & & \\
\hline $\mathrm{Bm}$ & $\begin{array}{l}\text { Lithocyclia } \\
\text { aristotelis group }\end{array}$ & & $\begin{array}{l}\text { Within } \\
32-4 \quad P \\
(283\end{array}$ & & & & \\
\hline $\mathrm{Bm}$ & $\begin{array}{l}\text { Carpocanistrum } \\
\text { azyx }\end{array}$ & & $\begin{array}{l}\text { Within } \\
32-4 \quad G \\
(283)\end{array}$ & & & & \\
\hline \multirow[t]{2}{*}{$\mathrm{Bm}$} & \multirow[t]{2}{*}{$\begin{array}{l}\text { Thyrsocyrtis } \\
\text { bromia }\end{array}$} & & \multirow{2}{*}{$\begin{array}{l}\text { Within } \\
32-4 \quad M \\
(283)\end{array}$} & & & & \\
\hline & & & & & & & \\
\hline
\end{tabular}


TABLE 9 - Continued

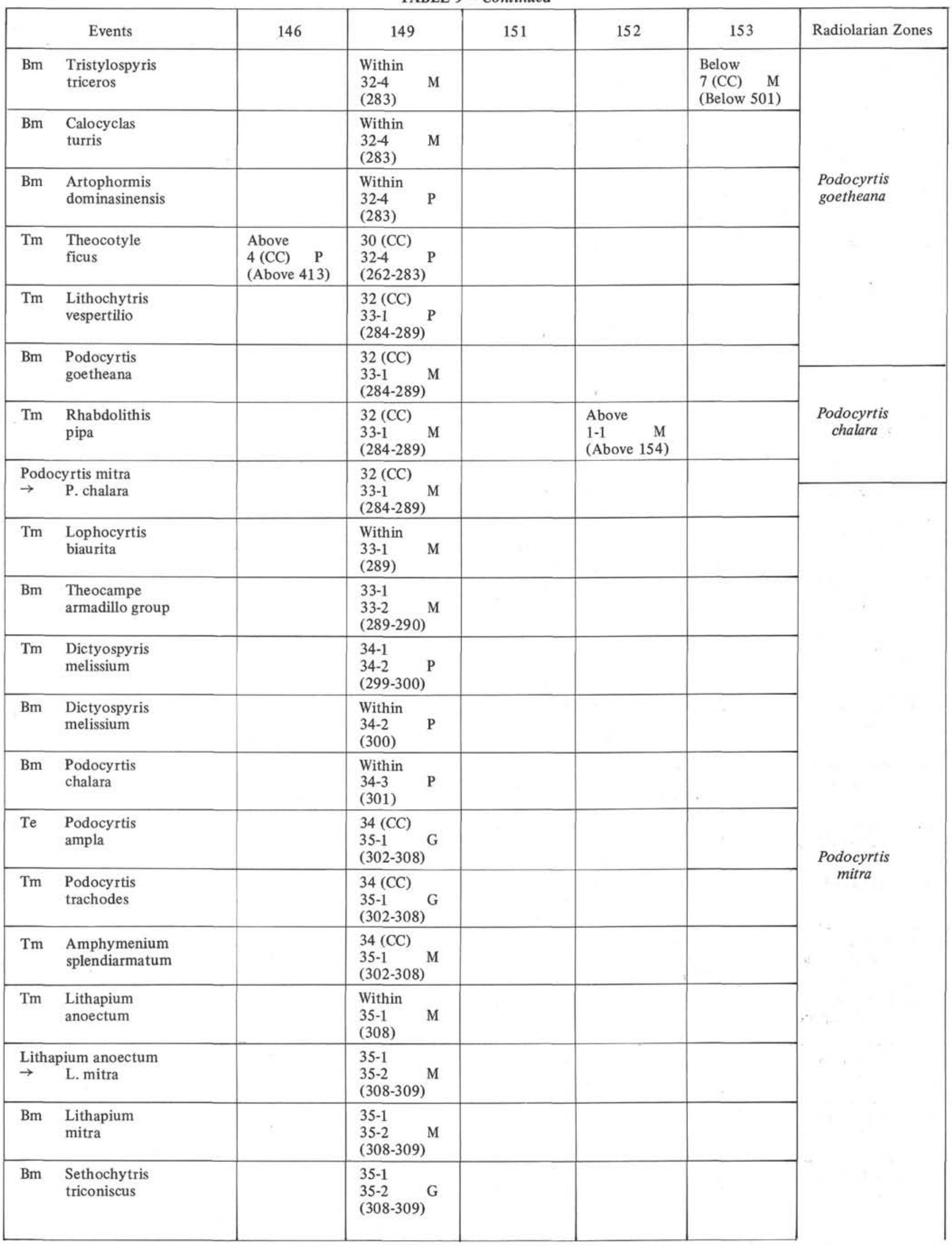


TABLE 9 - Continued

\begin{tabular}{|c|c|c|c|c|c|c|c|}
\hline & Events & 146 & 149 & 151 & 152 & 153 & Radiolarian Zones \\
\hline $\mathrm{Tm}$ & $\begin{array}{l}\text { Spongatractus } \\
\text { balbis }\end{array}$ & & $\begin{array}{l}35-2 \\
35-3 \quad P \\
(309-311)\end{array}$ & & & & \multirow{12}{*}{$\begin{array}{l}\text { Podocyrtis } \\
\text { mitra }\end{array}$} \\
\hline $\operatorname{Tm}$ & $\begin{array}{l}\text { Spongodiscus } \\
\text { quartus bosoculus }\end{array}$ & $\begin{array}{l}\text { Above } \\
6-1 \quad P \\
\text { (Above } 432 \text { ) }\end{array}$ & $\begin{array}{l}\text { Within } \\
35-5 \quad \mathrm{M} \\
(314)\end{array}$ & & & $\begin{array}{l}\text { Above } \\
9(\mathrm{CC}) \quad \mathrm{P} \\
\text { (Above 588) }\end{array}$ & \\
\hline $\operatorname{Tm}$ & $\begin{array}{l}\text { Spongodiscus } \\
\text { quartus quartus }\end{array}$ & $\begin{array}{l}\text { Above } \\
7-1 \quad \text { P } \\
\text { (Above } 441 \text { ) }\end{array}$ & & & $\begin{array}{l}\text { Above } \\
1-1\end{array}$ & & \\
\hline $\operatorname{Tm}$ & $\begin{array}{l}\text { Ceratospyris } \\
\text { articulata }\end{array}$ & & $\begin{array}{l}35-5 \\
35-6 \quad M \\
(314-315)\end{array}$ & & & & \\
\hline $\operatorname{Tm}$ & $\begin{array}{l}\text { Eusyringium } \\
\text { lagena }\end{array}$ & & $\begin{array}{l}35-6 \\
35(\mathrm{CC}) \quad \mathrm{M} \\
(315-316)\end{array}$ & & & & \\
\hline $\mathrm{Tm}$ & $\begin{array}{l}\text { Podocyrtis } \\
\text { sp. A }\end{array}$ & & $\begin{array}{l}35(\mathrm{CC}) \\
36-1 \quad \mathrm{M} \\
(316-317)\end{array}$ & & & & \\
\hline $\mathrm{Bm}$ & $\begin{array}{l}\text { Artophormis } \\
\text { barbadensis }\end{array}$ & & $\begin{array}{l}\text { Within } \\
36-1 \\
(317)\end{array}$ & & & & \\
\hline $\mathrm{Tm}$ & $\begin{array}{l}\text { Periphaena } \\
\text { tripyramis triangula }\end{array}$ & & $\begin{array}{l}36-1 \\
36(\mathrm{CC}) \quad P \\
(317)\end{array}$ & & & & \\
\hline $\operatorname{Tm}$ & $\begin{array}{l}\text { Lithochytris } \\
\text { archaea }\end{array}$ & & $\begin{array}{l}36(\mathrm{CC}) \\
37-1 \quad \mathrm{M} \\
(317-326)\end{array}$ & & & & \\
\hline $\mathrm{Bm}$ & $\begin{array}{l}\text { Podocyrtis } \\
\text { trachodes }\end{array}$ & & $\begin{array}{l}36(C C) \\
37-1 \quad M \\
(317-326)\end{array}$ & & & & \\
\hline $\operatorname{Tm}$ & $\begin{array}{l}\text { Podocyrtis } \\
\text { sinuosa }\end{array}$ & & $\begin{array}{l}35(\mathrm{CC}) \\
36(\mathrm{CC}) \quad \mathrm{M} \\
(316-317)\end{array}$ & & & & \\
\hline \multirow{2}{*}{\multicolumn{2}{|c|}{$\begin{array}{l}\text { Podocyrtis sinuosa } \\
\rightarrow \quad \text { P. mitra }\end{array}$}} & & $36(\mathrm{CC})$ & & & & \\
\hline & & & $\begin{array}{l}37-2 \quad G \\
(317-327)\end{array}$ & & & & \multirow{9}{*}{$\begin{array}{l}\text { Podocyrtis } \\
\text { ampla }\end{array}$} \\
\hline $\operatorname{Tm}$ & $\begin{array}{l}\text { Spongodiscus } \\
\text { americanus }\end{array}$ & $\begin{array}{l}\text { Above } \\
7-1 \quad M \\
\text { (Above }\end{array}$ & & & $\begin{array}{lr}\text { Above } \\
1-2\end{array}$ & $\begin{array}{l}\text { Above } \\
10(\text { CC) P } \\
\text { (Above 593) }\end{array}$ & \\
\hline $\operatorname{Tm}$ & $\begin{array}{l}\text { Lithapium } \\
\text { plegmacantha }\end{array}$ & & $\begin{array}{l}36(\mathrm{CC}) \\
37-1 \\
(317-326)\end{array}$ & & & & \\
\hline $\mathrm{Tm}$ & $\begin{array}{l}\text { Podocyrtis } \\
\text { sp. B }\end{array}$ & & $\begin{array}{l}36(\mathrm{CC}) \\
37-1 \quad \mathrm{G} \\
(317-326)\end{array}$ & & & & \\
\hline $\operatorname{Tm}$ & $\begin{array}{l}\text { Phormocyrtis } \\
\text { striata striata }\end{array}$ & $\begin{array}{l}\text { Above } \\
4 \text { (CC) P } \\
\text { (Above 413) }\end{array}$ & $\begin{array}{l}36(C C) \\
37-2 \quad P \\
(317-327)\end{array}$ & & & & \\
\hline \multicolumn{2}{|c|}{$\begin{array}{l}\text { Eusyringium lagena } \\
\rightarrow \quad \text { E. fistuligerum }\end{array}$} & & $\begin{array}{l}37-1 \\
37-4 \quad G \\
(326-329)\end{array}$ & & & & \\
\hline $\mathrm{Bm}$ & $\begin{array}{l}\text { Podocyrtis } \\
\text { sp. A. }\end{array}$ & & $\begin{array}{l}\text { Within } \\
37-4 \quad \text { M } \\
(329)\end{array}$ & & & & \\
\hline $\mathrm{Bm}$ & $\begin{array}{l}\text { Podocyrtis } \\
\text { mitra }\end{array}$ & & $\begin{array}{l}37-4 \\
37(\mathrm{CC}) \quad \mathrm{G} \\
(329-330)\end{array}$ & & & & \\
\hline $\mathrm{Bm}$ & $\begin{array}{l}\text { Podocyrtis } \\
\text { sp. B }\end{array}$ & & $\begin{array}{l}37-4 \\
37(\mathrm{CC}) \quad \mathrm{M} \\
(329-330)\end{array}$ & & & & \\
\hline
\end{tabular}


TABLE 9 - Continued

\begin{tabular}{|c|c|c|c|c|c|c|c|}
\hline & Events & 146 & 149 & 151 & 152 & 153 & Radiolarian Zones \\
\hline $\mathrm{Tm}$ & $\begin{array}{l}\text { Amphicraspedum } \\
\text { prolixum group }\end{array}$ & $\begin{array}{l}\text { Above } \\
6-1 \quad P \\
\text { (Above } 432 \text { ) }\end{array}$ & $\begin{array}{l}37(\mathrm{CC}) \\
38-3 \quad \mathrm{M} \\
(330-336)\end{array}$ & & $\begin{array}{l}\text { Above } \\
1-2 \quad G \\
\text { (Above 155) }\end{array}$ & & \multirow{7}{*}{$\begin{array}{l}\text { Podocyrtis } \\
\text { ampla }\end{array}$} \\
\hline $\operatorname{Tm}$ & $\begin{array}{l}\text { Podocyrtis } \\
\text { dorus }\end{array}$ & & $\begin{array}{l}38-3 \\
38(\mathrm{CC}) \quad \mathrm{M} \\
(336-337)\end{array}$ & & & ' & \\
\hline $\mathrm{Tm}$ & $\begin{array}{l}\text { Podocyrtis } \\
\text { diamesa }\end{array}$ & & $\begin{array}{l}38-3 \\
38(\mathrm{CC}) \quad \mathrm{M} \\
(336-337)\end{array}$ & & & & \\
\hline $\mathrm{Tm}$ & $\begin{array}{l}\text { Theocotyle } \\
\text { cryp to. cryptocephala }\end{array}$ & & $\begin{array}{l}38(\mathrm{CC}) \\
39-1 \quad \mathrm{M} \\
(337-344)\end{array}$ & & & & \\
\hline $\mathrm{Tm}$ & $\begin{array}{l}\text { Spongodiscus } \\
\text { phrix }\end{array}$ & & $\begin{array}{l}\begin{array}{l}38(\mathrm{CC}) \\
39-1 \quad \mathrm{M} \\
(337-344)\end{array} \\
\end{array}$ & & & & \\
\hline $\mathrm{Tm}$ & $\begin{array}{l}\text { Podocyrtis } \\
\text { phyxis }\end{array}$ & & $\begin{array}{l}38(\mathrm{CC}) \\
39-1 \quad M \\
(337-344)\end{array}$ & & & & \\
\hline \multirow{2}{*}{\multicolumn{2}{|c|}{$\begin{array}{l}\text { Podocyrtis phyxis } \\
\rightarrow \quad \text { P. ampla }\end{array}$}} & & \multirow{2}{*}{$\begin{array}{l}38(\mathrm{CC}) \\
39-1 \quad \mathrm{M} \\
(337-344)\end{array}$} & & & & \\
\hline & & & & & & & \multirow{14}{*}{$\begin{array}{l}\text { Thyrsocyrtis } \\
\text { triacantha }\end{array}$} \\
\hline $\mathrm{Bm}$ & $\begin{array}{l}\text { Podocyrtis } \\
\text { ampla }\end{array}$ & & $\begin{array}{l}38(\mathrm{CC}) \\
39-1 \quad \mathrm{M} \\
(337-344)\end{array}$ & & & & \\
\hline $\mathrm{Tm}$ & $\begin{array}{l}\text { Theocotyle } \\
\text { venezuelensis }\end{array}$ & & $\begin{array}{l}38(\mathrm{CC}) \\
39-1 \quad G \\
(337-344)\end{array}$ & & & & \\
\hline $\mathrm{Tm}$ & $\begin{array}{l}\text { Theocotyle } \\
\text { cryptocephala conica }\end{array}$ & & $\begin{array}{l}38(\mathrm{CC}) \\
39-1 \quad M \\
(337-344)\end{array}$ & & & & \\
\hline $\mathrm{Bm}$ & $\begin{array}{l}\text { Eusyringium } \\
\text { fistuligerum }\end{array}$ & & $\begin{array}{l}39-1 \\
39(\mathrm{CC}) \quad \mathrm{M} \\
(344-345)\end{array}$ & & & & \\
\hline \multicolumn{2}{|c|}{$\begin{array}{l}\text { Podocyrtis diamesa } \\
\rightarrow \quad \text { P.phyxis }\end{array}$} & & $\begin{array}{l}39-1 \\
40-1 \quad M \\
(344-353)\end{array}$ & & & & \\
\hline \multicolumn{2}{|c|}{$\begin{array}{l}\text { Lithochytris archaea } \\
\rightarrow \quad \text { L. vespertilio }\end{array}$} & & $\begin{array}{l}37-1 \\
42(\mathrm{CC}) \quad M \\
(326-376)\end{array}$ & & & & \\
\hline $\mathrm{Bm}$ & $\begin{array}{l}\text { Theocorys } \\
\text { anapographa }\end{array}$ & & $\begin{array}{l}\text { Within } \\
40-1 \quad M \\
(353)\end{array}$ & & & & \\
\hline $\mathrm{Bm}$ & $\begin{array}{l}\text { Podocyrtis } \\
\text { phyxis }\end{array}$ & & $\begin{array}{l}40-1 \\
40-2 \\
(353-354)\end{array}$ & & & & \\
\hline $\mathrm{Bm}$ & $\begin{array}{l}\text { Lithapium } \\
\text { anoectum }\end{array}$ & & $\begin{array}{l}40(\mathrm{CC}) \\
41-1 \quad \mathrm{M} \\
(355-363)\end{array}$ & & & & \\
\hline $\mathrm{Tm}$ & $\begin{array}{l}\text { Amphicraspedum } \\
\text { prolixum }\end{array}$ & $\begin{array}{l}\text { Above } \\
6(\text { CC) P } \\
\text { (Above 433) }\end{array}$ & $\begin{array}{l}40(\mathrm{CC}) \\
41-1 \quad \mathrm{M} \\
(355-363)\end{array}$ & & $\begin{array}{l}\text { Above } \\
1-2 \quad G \\
\text { (Above 155) }\end{array}$ & & \\
\hline Bm-e & $\begin{array}{l}\text { Podocyrtis } \\
\text { dorus }\end{array}$ & & $\begin{array}{l}41-1 \\
41-2 \quad M \\
(363-364)\end{array}$ & & & & \\
\hline $\operatorname{Tm}$ & $\begin{array}{l}\text { Theocorys } \\
\text { anaclasta }\end{array}$ & & $\begin{array}{l}41-5 \\
41-6 \quad M \\
(367-368)\end{array}$ & & & & \\
\hline $\mathrm{Tm}$ & $\begin{array}{l}\text { Thyrsocyrtis } \\
\text { hirsuta tensa }\end{array}$ & & $\begin{array}{l}\text { Within } \\
41-6 \quad \mathrm{M} \\
(368)\end{array}$ & & & & \\
\hline
\end{tabular}


TABLE 9 - Continued




TABLE 9 - Continued

\begin{tabular}{|c|c|c|c|c|c|c|c|}
\hline & Events & 146 & 149 & 151 & 152 & 153 & Radiolarian Zones \\
\hline Bm-e & $\begin{array}{l}\text { Podocyrtis } \\
\text { platypus }\end{array}$ & & $\begin{array}{l}42(\mathrm{CC}) \\
43-1 \quad M \\
(376-382)\end{array}$ & & & & \multirow{6}{*}{$\begin{array}{l}\text { Theocampe } \\
\text { mongolfieri }\end{array}$} \\
\hline $\mathrm{Bm}$ & $\begin{array}{l}\text { Lamptonium } \\
\text { fabae. constrictum }\end{array}$ & & $\begin{array}{l}42(\mathrm{CC}) \\
43-1 \quad P \\
(376-382)\end{array}$ & & & & \\
\hline $\mathrm{Bm}$ & $\begin{array}{l}\text { Theocorys } \\
\text { anaclasta }\end{array}$ & & $\begin{array}{l}42(\mathrm{CC}) \\
43-1 \quad P \\
(376-382)\end{array}$ & & & & \\
\hline $\mathrm{Bm}$ & $\begin{array}{l}\text { Dictyophimus } \\
\text { craticula }\end{array}$ & & $\begin{array}{l}42(\mathrm{CC}) \\
43-1 \quad \mathrm{M} \\
(376-382)\end{array}$ & & & & \\
\hline $\mathrm{Bm}$ & $\begin{array}{l}\text { Lithochytris } \\
\text { vespertilio }\end{array}$ & & $\begin{array}{l}42(\mathrm{CC}) \\
43-1 \quad \mathrm{M} \\
(376-382)\end{array}$ & & & & \\
\hline \multirow[t]{2}{*}{$\mathrm{Bm}$} & \multirow{2}{*}{$\begin{array}{l}\text { Theocampe } \\
\text { mongolfieri }\end{array}$} & & \multirow{2}{*}{$\begin{array}{l}\text { Below } \\
43-1 \\
\text { (Below }\end{array}$} & & & \multirow{2}{*}{$\begin{array}{l}\text { Below } \\
7 \text { (CC) G } \\
\text { (Below 501) }\end{array}$} & \\
\hline & & & & & & & \multirow{4}{*}{$\begin{array}{l}\text { Theocotyle } \\
\text { cryptocephala } \\
\text { cryptocephala }\end{array}$} \\
\hline $\mathrm{Bm}$ & $\begin{array}{l}\text { Podocyrtis } \\
\text { sinuosa }\end{array}$ & . & $\begin{array}{l}\text { Below } \\
43-1 \quad \text { G } \\
\text { (Below } 382 \text { ) }\end{array}$ & & & & \\
\hline $\mathrm{Bm}$ & $\begin{array}{l}\text { Thyrsocyrtis } \\
\text { hirsuta robusta }\end{array}$ & & $\begin{array}{l}\text { Below } \\
43-1 \quad M \\
\text { (Below }\end{array}$ & & & & \\
\hline \multirow[t]{2}{*}{$\mathrm{Bm}$} & \multirow{2}{*}{$\begin{array}{l}\text { Theocotyle } \\
\text { crypto. cryptocephala }\end{array}$} & & \multirow{2}{*}{$\begin{array}{l}\text { Below } \\
43-1 \quad \mathrm{M} \\
\text { (Below } \\
\end{array}$} & & & & \\
\hline & & & & & & & \multirow{5}{*}{$\begin{array}{l}\text { Phormocyrtis } \\
\text { striata striata }\end{array}$} \\
\hline $\mathrm{Bm}$ & $\begin{array}{l}\text { Rhopalocanium } \\
\text { ornatum }\end{array}$ & & $\begin{array}{l}\text { Below } \\
43-1 \quad \text { G } \\
\text { (Below } 382 \text { ) }\end{array}$ & & & & \\
\hline $\mathrm{Bm}$ & $\begin{array}{l}\text { Spongodiscus } \\
\text { phrix }\end{array}$ & & $\begin{array}{l}\text { Below } \\
43-1 \\
\text { (Below }\end{array}$ & & & & \\
\hline $\mathrm{Bm}$ & $\begin{array}{l}\text { Thyrsocyrtis } \\
\text { rhizodon }\end{array}$ & & $\begin{array}{l}\text { Below } \\
43-1 \quad M \\
\text { (Below } 382 \text { ) }\end{array}$ & & & & \\
\hline $\mathrm{Bm}$ & $\begin{array}{l}\text { Amphymenium } \\
\text { splendiarmatum }\end{array}$ & & $\begin{array}{l}\text { Below } \\
43-1 \quad P \\
\text { (Below }\end{array}$ & & & & \\
\hline $\mathrm{Bm}$ & $\begin{array}{l}\text { Phormocyrtis } \\
\text { striata striata }\end{array}$ & $\begin{array}{l}\text { Within } \\
7-1 \quad \text { M } \\
(441)\end{array}$ & $\begin{array}{l}\text { Below } \\
43-1 \quad M \\
\text { (Below }\end{array}$ & & & & \multirow{7}{*}{$\begin{array}{l}\text { Buryella } \\
\text { clinata }\end{array}$} \\
\hline $\mathrm{Bm}$ & $\begin{array}{l}\text { Theocampe } \\
\text { amphora group }\end{array}$ & & $\begin{array}{l}\text { Below } \\
\text { 43-1 } \quad \text { M } \\
\text { (Below } 382 \text { ) }\end{array}$ & & & , & \\
\hline $\mathrm{Tm}$ & $\begin{array}{l}\text { Dorcadospyris } \\
\text { confluens }\end{array}$ & & & & 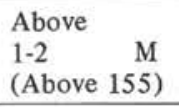 & & \\
\hline $\mathrm{Tm}$ & $\begin{array}{l}\text { Rhabdolithis } \\
\text { ellida }\end{array}$ & & & & $\begin{array}{l}\text { Above } \\
1-2 \\
\text { (Above } 155 \text { ) } \\
\end{array}$ & & \\
\hline $\mathrm{Bm}$ & $\begin{array}{l}\text { Lithocyclia } \\
\text { ocellus group }\end{array}$ & & $\begin{array}{l}\text { Below } \\
43-1 \quad G \\
\text { (Below }\end{array}$ & & & & \\
\hline $\mathrm{Bm}$ & $\begin{array}{l}\text { Spongatractus } \\
\text { pachystylus }\end{array}$ & & $\begin{array}{l}\text { Below } \\
43-1 \quad \mathrm{M} \\
\text { (Below }\end{array}$ & & . & & \\
\hline $\mathrm{Bm}$ & $\begin{array}{l}\text { Lithelius } \\
\text { hexaxyphophorus }\end{array}$ & & $\begin{array}{l}\text { Below } \\
43-1 \quad M \\
\text { (Below } 382 \text { ) }\end{array}$ & & & & \\
\hline
\end{tabular}


TABLE 9 - Continued

\begin{tabular}{|c|c|c|c|c|c|c|c|}
\hline & Events & 146 & 149 & 151 & 152 & 153 & Radiolarian Zones \\
\hline $\operatorname{Tm}$ & $\begin{array}{l}\text { Theocotyle } \\
\text { auctor }\end{array}$ & & & & $\begin{array}{l}\text { Above } \\
1-2 \\
\text { (Above } 155 \text { ) } \\
\end{array}$ & & \multirow{20}{*}{ 1 } \\
\hline $\mathrm{Bm}$ & $\begin{array}{l}\text { Ceratospyris } \\
\text { articulata }\end{array}$ & & $\begin{array}{lr}\text { Below } & \\
43-1 & M \\
\text { (Below 382) }\end{array}$ & & & & \\
\hline $\mathrm{Bm}$ & $\begin{array}{l}\text { Spongatractus } \\
\text { balbis }\end{array}$ & & $\begin{array}{lr}\text { Below } \\
43-1 & \mathrm{M} \\
\text { (Below } & 382 \text { ) } \\
\end{array}$ & & & & \\
\hline $\mathrm{Bm}$ & $\begin{array}{l}\text { Periphaena } \\
\text { decora }\end{array}$ & & $\begin{array}{lr}\text { Below } & \\
43-1 & M \\
\text { (Below } 382)\end{array}$ & & & $\begin{array}{l}\text { Below } \\
7 \text { (CC) M } \\
\text { (Below 501) }\end{array}$ & \\
\hline $\mathrm{Bm}$ & $\begin{array}{l}\text { Theocotyle } \\
\text { ficus }\end{array}$ & $\begin{array}{l}\text { Below } \\
4 \text { (CC) P } \\
\text { (Below } 413 \text { ) }\end{array}$ & $\begin{array}{lr}\text { Below } & \\
43-1 & \text { M } \\
\text { (Below } 382 \text { ) }\end{array}$ & & & & \\
\hline $\mathrm{Bm}$ & $\begin{array}{l}\text { Calocyclas } \\
\text { hispida }\end{array}$ & & $\begin{array}{l}\text { Below } \\
43-1 \\
\text { (Below } 382 \text { ) } \\
\end{array}$ & & & & \\
\hline $\mathrm{Tm}$ & $\begin{array}{l}\text { Theocorys (?) } \\
\text { phyzella }\end{array}$ & 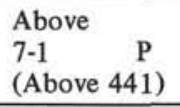 & & & $\begin{array}{l}\text { Above } \\
1-2 \quad M \\
\text { (Above } 155 \text { ) }\end{array}$ & & \\
\hline $\mathrm{Tm}$ & $\begin{array}{l}\text { Pterocodon } \\
\text { tenellus }\end{array}$ & $?$ & & & $\begin{array}{l}\text { Above } \\
1-1\end{array}$ & & \\
\hline $\mathrm{Bm}$ & $\begin{array}{l}\text { Lithochytris } \\
\text { archaea }\end{array}$ & & $\begin{array}{l}\text { Below } \\
\text { 43-1 M } \\
\text { (Below 382) }\end{array}$ & & & & \\
\hline $\mathrm{Bm}$ & $\begin{array}{l}\text { Lophocyrtis } \\
\text { biaurita }\end{array}$ & & $\begin{array}{lr}\text { Below } & \\
43-1 & \text { M } \\
\text { (Below } & 382)\end{array}$ & & & & \\
\hline $\mathrm{Bm}$ & $\begin{array}{l}\text { Theocampe } \\
\text { urceolus }\end{array}$ & & $\begin{array}{l}\text { Below } \\
\text { 43-1 M } \\
\text { (Below 382) }\end{array}$ & & & & \\
\hline $\mathrm{Tm}$ & $\begin{array}{l}\text { Astrosphaerin } \\
\text { sp. C }\end{array}$ & & & & 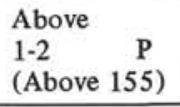 & & \\
\hline $\mathrm{Bm}$ & $\begin{array}{l}\text { Lamptonium } \\
\text { fabae. chaunothorax }\end{array}$ & & $\begin{array}{lr}\text { Below } & \\
43-1 & \mathrm{M} \\
\text { (Below } & 382 \text { ) }\end{array}$ & & & & \\
\hline $\mathrm{Bm}$ & $\begin{array}{l}\text { Theocotyle } \\
\text { venezuelensis }\end{array}$ & & $\begin{array}{lr}\text { Below } \\
43-1 \\
\text { (Below } 382 \text { ) } \\
\end{array}$ & & & & \\
\hline $\mathrm{Tm}$ & $\begin{array}{l}\text { Phormocyrtis } \\
\text { striata exquisita }\end{array}$ & $\begin{array}{l}4(\mathrm{CC}) \\
6-1 \quad M \\
(413-432)\end{array}$ & & & $\begin{array}{l}\text { Above } \\
1-1 \quad \text { G } \\
\text { (Above 154) }\end{array}$ & $\begin{array}{l}\text { Above } \\
9(\mathrm{CC}) \quad \mathrm{M} \\
\text { (Above 588) }\end{array}$ & \\
\hline $\mathrm{Tm}$ & $\begin{array}{l}\text { Phormocyrtis } \\
\text { turgida }\end{array}$ & $\begin{array}{l}\text { Above } \\
\text { 6-1 } \quad P \\
\text { (Above } 432 \text { ) }\end{array}$ & & & $\begin{array}{l}\text { Above } \\
1-1\end{array}$ & & \\
\hline $\operatorname{Tm}$ & $\begin{array}{l}\text { Buryella } \\
\text { tetradica }\end{array}$ & $\begin{array}{l}4(\mathrm{CC}) \\
6-1 \quad \mathrm{M} \\
(413-432)\end{array}$ & & & $\begin{array}{l}\text { Above } \\
1-1 \\
\text { (Above } 154 \text { ) } \\
\end{array}$ & $\begin{array}{l}\text { Above } \\
9 \text { (CC) G } \\
\text { (Above 588) }\end{array}$ & \\
\hline $\mathrm{Tm}$ & $\begin{array}{l}\text { Lychnocanoma } \\
\text { auxilla }\end{array}$ & $\begin{array}{l}\text { Above } \\
6(\text { CC) P } \\
\text { (Above 433) }\end{array}$ & & & $\begin{array}{l}\text { Above } \\
1-2 \quad M \\
\text { (Above } 155 \text { ) } \\
\end{array}$ & $\begin{array}{l}\text { Above } \\
9(\text { CC) } \quad \text { P } \\
\text { (Above 588) }\end{array}$ & \\
\hline $\mathrm{Tm}$ & $\begin{array}{l}\text { Dendrospyris } \\
\text { fragoides }\end{array}$ & $\begin{array}{l}\text { Above } \\
7-1 \quad M \\
\text { (Above } 441 \text { ) }\end{array}$ & & & $\begin{array}{l}\text { Above } \\
1-1 \\
\text { (Above } 154 \text { ) }\end{array}$ & & \\
\hline $\mathrm{Tm}$ & $\begin{array}{l}\text { Stylotrochus } \\
\text { nitidus }\end{array}$ & $\begin{array}{l}\text { Above } \\
7-1 \quad M \\
\text { (Above } 441 \text { ) }\end{array}$ & & & $\begin{array}{l}\text { Above } \\
1-2 \quad G \\
\text { (Above 155) }\end{array}$ & & \\
\hline
\end{tabular}


TABLE 9 - Continued

\begin{tabular}{|c|c|c|c|c|c|c|c|}
\hline & Events & 146 & 149 & 151 & 152 & 153 & \multirow{7}{*}{ Radiolarian Zones } \\
\hline $\mathrm{Tm}$ & $\begin{array}{l}\text { Theocorys } \\
\text { acroria }\end{array}$ & $\begin{array}{l}\text { Above } \\
7-1 \quad P \\
\text { (Above } 441 \text { ) } \\
\end{array}$ & & & $\begin{array}{l}\text { Above } \\
1-2 \quad M \\
\text { (Above } 155 \text { ) }\end{array}$ & & \\
\hline $\mathrm{Tm}$ & $\begin{array}{l}\text { Phormocyrtis } \\
\text { cubensis }\end{array}$ & $\begin{array}{l}\text { Above } \\
7-1 \quad M \\
\text { (Above } 441 \text { ) }\end{array}$ & & & $\begin{array}{l}\text { Above } \\
1-1 \quad G \\
\text { (Above } 154 \text { ) }\end{array}$ & & \\
\hline $\mathrm{Tm}$ & $\begin{array}{l}\text { Giraffospyris } \\
\text { lata }\end{array}$ & $\begin{array}{l}\text { Above } \\
7-1 \quad M \\
\text { (Above } 441 \text { ) } \\
\end{array}$ & & & $\begin{array}{l}\text { Above } \\
1-1 \quad G \\
\text { (Above } 154 \text { ) }\end{array}$ & & \\
\hline $\mathrm{Tm}$ & $\begin{array}{l}\text { Thecosphaera } \\
\text { larnacium }\end{array}$ & $\begin{array}{l}\text { Above } \\
7-1 \quad P \\
\text { (Above } 441 \text { ) }\end{array}$ & & & $\begin{array}{lr}\text { Above } & \\
1-2 & M \\
\text { (Above } 155 \text { ) }\end{array}$ & & \\
\hline $\mathrm{Tm}$ & $\begin{array}{l}\text { Spongomelissa } \\
\text { adunca }\end{array}$ & $\begin{array}{l}\text { Above } \\
7-1 \quad P \\
\text { (Above } 441 \text { ) }\end{array}$ & & & $\begin{array}{l}\text { Above } \\
1-2 \quad M \\
\text { (Above } 155 \text { ) }\end{array}$ & & \\
\hline $\mathrm{Bm}$ & $\begin{array}{l}\text { Buryella } \\
\text { clinata }\end{array}$ & $\begin{array}{l}\text { Below } \\
4 \text { (CC) M } \\
\text { (Below 413) } \\
\end{array}$ & & & & & \\
\hline $\mathrm{Tm}$ & $\begin{array}{l}\text { Thyrsocyrtis } \\
\text { tarsipes }\end{array}$ & $\begin{array}{l}\text { Above } \\
7-1 \quad P \\
\text { (Above } 441 \text { ) }\end{array}$ & & & $\begin{array}{l}\text { Above } \\
1-2 \quad M \\
\text { (Above } 155 \text { ) }\end{array}$ & & \\
\hline $\mathrm{Tm}$ & $\begin{array}{l}\text { Lamptonium } \\
\text { pennatum }\end{array}$ & $\begin{array}{l}\text { Above } \\
\text { (Above 441) }\end{array}$ & & & $\begin{array}{l}\text { Above } \\
\text { (Above 155) }\end{array}$ & & \\
\hline $\mathrm{Tm}$ & $\begin{array}{l}\text { Pterocodon (?) } \\
\text { ampla (?) }\end{array}$ & $\begin{array}{l}\text { Above } \\
7-1 \quad P \\
\text { (Above } 441 \text { ) }\end{array}$ & & & $\begin{array}{l}\text { Above } \\
1-2 \quad M \\
\text { (Above } 155 \text { ) }\end{array}$ & & \\
\hline $\mathrm{Tm}$ & $\begin{array}{l}\text { Amphisphaera } \\
\text { minor }\end{array}$ & $\begin{array}{l}\text { Above } \\
7-1 \quad P \\
\text { (Above } 441 \text { ) }\end{array}$ & & & $\begin{array}{l}\text { Above } \\
1-2 \quad M \\
\text { (Above } 155 \text { ) }\end{array}$ & & \\
\hline $\mathrm{Tm}$ & $\begin{array}{l}\text { Astrosphaerin } \\
\text { sp. E }\end{array}$ & $\begin{array}{l}\text { Above } \\
10-2 \quad P \\
\text { (Above 469) }\end{array}$ & & & $\begin{array}{l}\text { Above } \\
1-2 \quad M \\
\text { (Above } 155 \text { ) }\end{array}$ & & \\
\hline $\mathrm{Tm}$ & $\begin{array}{l}\text { Astrosphaerin } \\
\text { sp. D }\end{array}$ & $\begin{array}{l}\text { Above } \\
10-2 \quad P \\
\text { (Above } 469 \text { ) }\end{array}$ & & & $\begin{array}{l}\text { Above } \\
1-2 \quad P \\
\text { (Above } 155 \text { ) }\end{array}$ & & \\
\hline $\mathrm{Tm}$ & $\begin{array}{l}\text { Stylosphaera } \\
\text { coronata sabaca }\end{array}$ & $\begin{array}{l}\text { Above } \\
7-1 \quad P \\
\text { (Above } 441 \text { ) }\end{array}$ & & & $\begin{array}{l}1-2 \\
2-4 \\
(155-166)\end{array}$ & & Bekoma \\
\hline $\mathrm{Tm}$ & $\begin{array}{l}\text { Axoprunum } \\
\text { pierinae group }\end{array}$ & $\begin{array}{l}\text { Above } \\
4 \text { (CC) M } \\
\text { (Above 413) }\end{array}$ & & & $\begin{array}{l}\text { Above } \\
2-4 \quad M \\
\text { (Above } 166 \text { ) }\end{array}$ & & \\
\hline $\mathrm{Tm}$ & $\begin{array}{l}\text { Dictyospyris } \\
\text { discus }\end{array}$ & $\begin{array}{l}\text { Above } \\
7-1 \quad P \\
\text { (Above } 441 \text { ) }\end{array}$ & & & $\begin{array}{l}\text { Above } \\
2-4\end{array}$ & & \\
\hline $\operatorname{Tm}$ & $\begin{array}{l}\text { Stylosphaera } \\
\text { goruna }\end{array}$ & $\begin{array}{l}\text { Above } \\
7-1 \quad P \\
\text { (Above } 441 \text { ) }\end{array}$ & & & $\begin{array}{l}\text { Within } \\
2-4 \\
(166)\end{array}$ & $\begin{array}{l}\text { Above } \\
9 \text { (CC) M } \\
\text { (Above 588) }\end{array}$ & \\
\hline $\mathrm{Bm}$ & $\begin{array}{l}\text { Theocorys (?) } \\
\text { phyzella }\end{array}$ & $\begin{array}{l}\text { Below } \\
7 \text { (CC) P } \\
\text { (Below 442) }\end{array}$ & & & $\begin{array}{l}2-4 \\
2-5 \quad P \\
(166-167)\end{array}$ & & \\
\hline $\mathrm{Bm}$ & $\begin{array}{l}\text { Theocotyle } \\
\text { auctor }\end{array}$ & & & & $\begin{array}{l}2-4 \\
2-5 \quad M \\
(166-167)\end{array}$ & & \\
\hline $\mathrm{Tm}$ & $\begin{array}{l}\text { Stylotrochus } \\
\text { alveatus }\end{array}$ & $\begin{array}{l}\text { Above } \\
7-1 \quad \text { M } \\
\text { (Above 441) }\end{array}$ & & & $\begin{array}{l}\text { Above } \\
2-5 \\
\text { (Above 167) }\end{array}$ & $\begin{array}{l}\text { Above } \\
9 \text { (CC) M } \\
\text { (Above 588) }\end{array}$ & \\
\hline $\mathrm{Bm}$ & $\begin{array}{l}\text { Dorcadospyris } \\
\text { confluens }\end{array}$ & & & & $\begin{array}{l}2-4 \\
2(\mathrm{CC}) \quad M \\
(166-168)\end{array}$ & & \\
\hline
\end{tabular}


TABLE 9 - Continued

\begin{tabular}{|c|c|c|c|c|c|c|c|}
\hline & Events & 146 & 149 & 151 & 152 & 153 & Radiolarian Zones \\
\hline $\mathrm{Bm}$ & $\begin{array}{l}\text { Stylosphaera } \\
\text { coronata sabaca }\end{array}$ & $\begin{array}{l}\text { Below } \\
7-1 \quad P \\
\text { (Below } 441)\end{array}$ & & & $\begin{array}{l}3-4 \\
3-5 \\
(176-177)\end{array}$ & & \multirow{4}{*}{$\begin{array}{c}\text { Bekoma } \\
\text { bidarfensis }\end{array}$} \\
\hline $\mathrm{Tm}$ & $\begin{array}{l}\text { Xiphospira } \\
\text { circularis }\end{array}$ & $\begin{array}{l}\text { Above } \\
7-1 \\
\text { (Above }\end{array}$ & & & $\begin{array}{l}\text { Above } \\
3-5 \quad G \\
\text { (Above 177) }\end{array}$ & & \\
\hline $\mathrm{Tm}$ & $\begin{array}{l}\text { Amphicraspedum } \\
\text { murrayanum }\end{array}$ & $\begin{array}{l}\text { Above } \\
7-1 \quad P \\
\text { (Above } 441 \text { ) }\end{array}$ & & & $\begin{array}{l}\text { Above } \\
3-5 \quad P \\
\text { (Above } 177 \text { ) }\end{array}$ & & \\
\hline $\mathrm{Tm}$ & $\begin{array}{l}\text { Astrosphaerin } \\
\text { sp. F }\end{array}$ & $\begin{array}{l}\text { Above } \\
10-2 \quad P \\
\text { (Above } 469 \text { ) }\end{array}$ & & & $\begin{array}{l}\text { Above } \\
3-5 \quad M \\
\text { (Above 177) }\end{array}$ & & \\
\hline $\mathrm{Bm}$ & $\begin{array}{l}\text { Amphicraspedum } \\
\text { murrayanum }\end{array}$ & $\begin{array}{l}\text { Below } \\
7-1 \quad P \\
\text { (Below } 441)\end{array}$ & & & $\begin{array}{l}\text { Below } \\
3-5 \quad P \\
\text { (Below 177) }\end{array}$ & & \multirow{12}{*}{ 更 } \\
\hline $\mathrm{Bm}$ & $\begin{array}{l}\text { Astrosphaerin } \\
\text { sp. C }\end{array}$ & & & & $\begin{array}{l}\text { Below } \\
3-5 \quad \text { M } \\
\text { (Below 177) }\end{array}$ & & \\
\hline $\mathrm{Bm}$ & $\begin{array}{l}\text { Spongomelissa } \\
\text { adunca }\end{array}$ & $\begin{array}{l}\text { Below } \\
7-1 \quad \text { P } \\
\text { (Below } 441)\end{array}$ & & & $\begin{array}{l}\text { Below } \\
3(\mathrm{CC}) \quad \mathrm{M} \\
\text { (Below 178) }\end{array}$ & & \\
\hline $\mathrm{Bm}$ & $\begin{array}{l}\text { Astrosphaerin } \\
\text { sp. E }\end{array}$ & $\begin{array}{l}\text { Below } \\
10-2 \quad P \\
\text { (Below } 469)\end{array}$ & & & $\begin{array}{l}\text { Below } \\
3 \text { (CC) M } \\
\text { (Below 178) }\end{array}$ & & \\
\hline $\mathrm{Tm}$ & $\begin{array}{l}\text { Lithelius } \\
\text { foremanae }\end{array}$ & $\begin{array}{l}\text { Above } \\
7-1 \quad P \\
\text { (Above } 441 \text { ) }\end{array}$ & & & $\begin{array}{l}\text { Above } \\
4-2 \quad M \\
\text { (Above 184) }\end{array}$ & & \\
\hline \multicolumn{2}{|c|}{$\begin{array}{l}\text { Lamptonium pennatum } \\
\rightarrow \quad \text { L. fabae. fabaeforme }\end{array}$} & & & & $\begin{array}{l}1-2 \\
4(\mathrm{CC}) \quad \mathrm{M} \\
(155-186)\end{array}$ & & \\
\hline $\mathrm{Bm}$ & $\begin{array}{l}\text { Lamptonium } \\
\text { fabae. fabaeforme }\end{array}$ & $\begin{array}{l}\text { Below } \\
7-1 \quad P \\
\text { (Below } 441 \text { ) } \\
\end{array}$ & \multirow[t]{2}{*}{$\begin{array}{l}42-4 \\
42(\mathrm{CC}) \quad P \\
(375-376)\end{array}$} & & $\begin{array}{l}4-2 \\
4(C C) \quad G \\
(184-186)\end{array}$ & & \\
\hline $\mathrm{Bm}$ & $\begin{array}{l}\text { Lychnocanoma } \\
\text { sp. aff. L. bellum }\end{array}$ & & & & $\begin{array}{l}4-2 \\
6-5 \quad M \\
(184-207)\end{array}$ & & \\
\hline $\mathrm{Bm}$ & $\begin{array}{l}\text { Lithomitra } \\
\text { docilis }\end{array}$ & & $\begin{array}{l}\text { Below } \\
42 \text { (CC) M } \\
\text { (Below 376) }\end{array}$ & & $\begin{array}{l}4-2 \\
6-5 \quad M \\
(184-207)\end{array}$ & & \\
\hline $\operatorname{Tm}$ & $\begin{array}{l}\text { Clathrocycloma } \\
\text { parcum }\end{array}$ & & & & $\begin{array}{l}4-2 \\
6-5 \quad M \\
(184-207)\end{array}$ & & \\
\hline $\mathrm{Bm}$ & $\begin{array}{l}\text { Thyrsocyrtis } \\
\text { tarsipes }\end{array}$ & $\begin{array}{l}\text { Below } \\
7-1 \quad P \\
\text { (Below } 441 \text { ) }\end{array}$ & & & $\begin{array}{l}4-2 \\
6-5 \quad P \\
(184-207)\end{array}$ & & \\
\hline $\mathrm{Bm}$ & $\begin{array}{l}\text { Pterocodon } \\
\text { tenellus }\end{array}$ & $?$ & & & $\begin{array}{l}4-2 \\
6-5 \quad M \\
(184-207)\end{array}$ & & \\
\hline $\mathrm{Bm}$ & $\begin{array}{l}\text { Dictyospyris } \\
\text { discus }\end{array}$ & 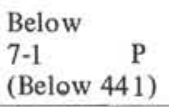 & & & $\begin{array}{l}\text { Below } \\
4-2 \quad \text { G } \\
\text { (Below 184) }\end{array}$ & & \multirow{4}{*}{ Unzoned } \\
\hline $\mathrm{Bm}$ & $\begin{array}{l}\text { Rhabdolithis } \\
\text { pipa }\end{array}$ & & $\begin{array}{l}\text { Below } \\
42(\mathrm{CC}) \quad \mathrm{M} \\
\text { (Below 376) }\end{array}$ & & $\begin{array}{l}\text { Below } \\
4-2 \quad M \\
\text { (Below } 184 \text { ) }\end{array}$ & & \\
\hline $\mathrm{Bm}$ & $\begin{array}{l}\text { Rhabdolithis } \\
\text { ellida }\end{array}$ & & & & $\begin{array}{l}\text { Below } \\
4-2 \quad M \\
\text { (Below } 184)\end{array}$ & & \\
\hline $\mathrm{Bm}$ & $\begin{array}{l}\text { Carposphaera } \\
\text { subbotinae }\end{array}$ & & & & $\begin{array}{l}\text { Below } \\
4-2 \quad P \\
\text { (Below } 184 \text { ) }\end{array}$ & & \\
\hline
\end{tabular}


TABLE 9 - Continued

\begin{tabular}{|c|c|c|c|c|c|c|c|}
\hline & Events & 146 & 149 & 151 & 152 & 153 & Radiolarian Zones \\
\hline $\mathrm{Bm}$ & $\begin{array}{l}\text { Phormocyrtis } \\
\text { turgida }\end{array}$ & 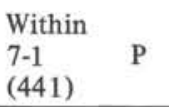 & & & $\begin{array}{l}4(\mathrm{CC}) \\
6-5 \quad M \\
(186-207)\end{array}$ & & \multirow{20}{*}{ Unzoned } \\
\hline $\mathrm{Bm}$ & $\begin{array}{l}\text { Podocyrtis } \\
\text { papalis }\end{array}$ & $\begin{array}{l}\text { Below } \\
7-1 \quad P \\
\text { (Below } 441 \text { ) }\end{array}$ & $\begin{array}{l}41-4 \\
41-6 \quad M \\
(366-368)\end{array}$ & & $\begin{array}{l}4(\mathrm{CC}) \\
6-5 \quad \mathrm{G} \\
(186-207)\end{array}$ & & \\
\hline $\mathrm{Bm}$ & $\begin{array}{l}\text { Theocorys } \\
\text { acroria }\end{array}$ & $\begin{array}{l}\text { Below } \\
7-1 \\
\text { (Below } 441)\end{array}$ & & & $\begin{array}{l}4(\mathrm{CC}) \\
6-5 \quad M \\
(186-207)\end{array}$ & & \\
\hline $\mathrm{Bm}$ & $\begin{array}{l}\text { Lychnocanoma } \\
\text { auxilla }\end{array}$ & $\begin{array}{l}\text { Below } \\
7-1 \\
\text { (Below }\end{array}$ & & & $\begin{array}{l}4(\mathrm{CC}) \\
6-5 \quad M \\
(186-207)\end{array}$ & $\begin{array}{l}\text { Below } \\
9 \text { (CC) P } \\
\text { (Below 588) }\end{array}$ & \\
\hline $\mathrm{Tm}$ & $\begin{array}{l}\text { Buryella } \\
\text { pentadica }\end{array}$ & $\begin{array}{l}7-1 \\
7(\mathrm{CC}) \quad P \\
(441-442)\end{array}$ & & & $\begin{array}{l}4(\mathrm{CC}) \\
6-5 \quad \mathrm{G} \\
(186-207)\end{array}$ & $\begin{array}{l}\text { Above } \\
9(\text { CC) G } \\
\text { (Above 588) }\end{array}$ & \\
\hline $\mathrm{Bm}$ & $\begin{array}{l}\text { Phormocyrtis } \\
\text { cubensis }\end{array}$ & $\begin{array}{l}\text { Below } \\
7-1 \quad P \\
\text { (Below } 441 \text { ) }\end{array}$ & & & $\begin{array}{l}4(\mathrm{CC}) \\
6-5 \quad M \\
(186-207)\end{array}$ & & \\
\hline $\mathrm{Bm}$ & $\begin{array}{l}\text { Stylotrochus } \\
\text { nitidus }\end{array}$ & $\begin{array}{l}\text { Below } \\
7-1 \\
\text { (Below } 441 \text { ) }\end{array}$ & & & $\begin{array}{l}4(\mathrm{CC}) \\
6-5 \quad M \\
(186-207)\end{array}$ & & \\
\hline $\mathrm{Bm}$ & $\begin{array}{l}\text { Spongodiscus } \\
\text { quartus quartus }\end{array}$ & $\begin{array}{l}\text { Below } \\
7-1 \\
\text { (Below } 441 \text { ) }\end{array}$ & & & $\begin{array}{l}4(\mathrm{CC}) \\
6-5 \quad M \\
(186-207)\end{array}$ & & \\
\hline $\mathrm{Bm}$ & $\begin{array}{l}\text { Lychnocanoma } \\
\text { babylonis group }\end{array}$ & $\begin{array}{l}\text { Below } \\
7 \text { (CC) M } \\
\text { (Below 442) }\end{array}$ & $\begin{array}{l}\text { Below } \\
43-1 \quad M \\
\text { (Below }\end{array}$ & & $\begin{array}{l}4(\mathrm{CC}) \\
6-5 \quad \mathrm{G} \\
(186-207)\end{array}$ & & \\
\hline $\mathrm{Bm}$ & $\begin{array}{l}\text { Dendrospyris } \\
\text { fragoides }\end{array}$ & $\begin{array}{l}\text { Below } \\
7-1 \quad \text { M } \\
\text { (Below } 441 \text { ) }\end{array}$ & & & $\begin{array}{l}\text { Below } \\
4 \text { (CC) G } \\
\text { (Below 186) }\end{array}$ & & \\
\hline $\mathrm{Bm}$ & $\begin{array}{l}\text { Giraffospyris } \\
\text { lata }\end{array}$ & $\begin{array}{ll}\text { Below } & \\
7-1 & M \\
\text { (Below } & 441)\end{array}$ & & & $\begin{array}{l}\text { Below } \\
4(C C) \quad M \\
\text { (Below 186) }\end{array}$ & & \\
\hline $\mathrm{Bm}$ & $\begin{array}{l}\text { Amphicraspedum } \\
\text { prolixum }\end{array}$ & $\begin{array}{l}\text { Below } \\
7-1 \quad P \\
\text { (Below } 441 \text { ) }\end{array}$ & $\begin{array}{l}\text { Below } \\
43-1 \quad \text { G } \\
\text { (Below } 382 \text { ) }\end{array}$ & & $\begin{array}{l}\text { Below } \\
4(\text { CC) M } \\
\text { (Below 186) }\end{array}$ & & \\
\hline $\mathrm{Bm}$ & $\begin{array}{l}\text { Amphicraspedum } \\
\text { prolixum group }\end{array}$ & $\begin{array}{l}\text { Below } \\
7-1 \quad M \\
\text { (Below } 441 \text { ) }\end{array}$ & $\begin{array}{l}\text { Below } \\
43-1 \quad \text { G } \\
\text { (Below 382) }\end{array}$ & & $\begin{array}{l}\text { Below } \\
4 \text { (CC) M } \\
\text { (Below 186) }\end{array}$ & & \\
\hline $\mathrm{Tm}$ & $\begin{array}{l}\text { Clathrocycloma } \\
\text { capitaneum }\end{array}$ & $\begin{array}{l}9-2 \\
10-2 \quad P \\
(460-469)\end{array}$ & & & $\begin{array}{l}\text { Within } \\
6-5 \\
(207)\end{array}$ & & \\
\hline $\mathrm{Tm}$ & $\begin{array}{l}\text { Bekoma } \\
\text { campechensis }\end{array}$ & & & & $\begin{array}{l}6-5 \\
7-3 \quad P \\
(207-214)\end{array}$ & & \\
\hline $\mathrm{Tm}$ & $\begin{array}{l}\text { Bekoma (?) } \\
\text { demissa }\end{array}$ & $\begin{array}{l}\text { Above } \\
10-2 \\
\text { (Above 469) }\end{array}$ & & & $\begin{array}{l}6-5 \\
7-3 \quad M \\
(207-214)\end{array}$ & & \\
\hline $\mathrm{Tm}$ & $\begin{array}{l}\text { Bekoma } \\
\text { divaricata }\end{array}$ & $\begin{array}{l}\text { Above } \\
7-1 \quad P \\
\text { (Above } 441 \text { ) }\end{array}$ & & & $\begin{array}{l}6-5 \\
7-3 \quad P \\
(207-214)\end{array}$ & & \\
\hline $\mathrm{Tm}$ & $\begin{array}{l}\text { Hexacontium } \\
\text { palaeocenicum }\end{array}$ & $\begin{array}{l}\text { Above } \\
7-1 \quad P \\
\text { (Above } 441 \text { ) }\end{array}$ & & & $\begin{array}{l}6-5 \\
7-4 \quad M \\
(207-215)\end{array}$ & & \\
\hline $\mathrm{Bm}$ & $\begin{array}{l}\text { Thecosphaera } \\
\text { larnacium }\end{array}$ & $\begin{array}{l}\text { Below } \\
7-1 \quad P \\
\text { (Below } 441 \text { ) }\end{array}$ & & & $\begin{array}{l}6-5 \\
7-4 \quad P \\
(207-215)\end{array}$ & & \\
\hline $\mathrm{Bm}$ & $\begin{array}{l}\text { Thecosphaerella } \\
\text { ptomatus }\end{array}$ & & $\begin{array}{l}\text { Below } \\
43-1 \quad M \\
\text { (Below } 382 \text { ) }\end{array}$ & & $\begin{array}{l}6-5 \\
7-4 \quad P \\
(207-215)\end{array}$ & & \\
\hline
\end{tabular}


TABLE 9 - Continued

\begin{tabular}{|c|c|c|c|c|c|c|c|}
\hline & Events & 146 & 149 & 151 & 152 & 153 & Radiolarian Zones \\
\hline $\mathrm{Bm}$ & $\begin{array}{l}\text { Lithelius } \\
\text { foremanae }\end{array}$ & $\begin{array}{l}\text { Below } \\
7-1 \quad M \\
\text { (Below } 441)\end{array}$ & & & $\begin{array}{l}\text { Below } \\
6-5 \quad M \\
\text { (Below 207) }\end{array}$ & & \multirow{20}{*}{ Unzoned } \\
\hline $\mathrm{Bm}$ & $\begin{array}{l}\text { Bekoma } \\
\text { divaricata }\end{array}$ & $\begin{array}{l}\text { Below } \\
7-1 \quad P \\
\text { (Below } 441 \text { ) }\end{array}$ & & & $\begin{array}{l}\text { Below } \\
7-3 \\
\text { (Below } 214 \text { ) }\end{array}$ & & \\
\hline $\mathrm{Bm}$ & $\begin{array}{l}\text { Astrosphaerin } \\
\text { sp. D }\end{array}$ & $\begin{array}{l}\text { Below } \\
10-2 \quad \text { G } \\
\text { (Below 469) }\end{array}$ & & & $\begin{array}{l}\text { Below } \\
7-3 \quad \text { P } \\
\text { (Below } 214 \text { ) }\end{array}$ & & \\
\hline $\mathrm{Bm}$ & $\begin{array}{l}\text { Astrosphaerin } \\
\text { sp. F }\end{array}$ & $\begin{array}{l}\text { Below } \\
10-2 \quad M \\
\text { (Below } 469 \text { ) }\end{array}$ & & & $\begin{array}{l}\text { Below } \\
7-3 \\
\text { (Below } 214 \text { ) }\end{array}$ & & \\
\hline $\mathrm{Tm}$ & $\begin{array}{l}\text { Lamptonium } \\
\text { incohatum }\end{array}$ & $\begin{array}{l}\text { Above } \\
9-2 \quad P \\
\text { (Above } 460 \text { ) }\end{array}$ & & & $?$ & $\begin{array}{lr}\text { Above } & \\
11-1 & M \\
\text { (Above } & 601 \text { ) }\end{array}$ & \\
\hline $\mathrm{Bm}$ & $\begin{array}{l}\text { Xiphospira } \\
\text { circularis }\end{array}$ & $\begin{array}{l}\text { Below } \\
7-1 \quad M \\
\text { (Below } 441 \text { ) }\end{array}$ & & & $\begin{array}{l}\text { Below } \\
7-4 \\
\text { (Below } 215 \text { ) }\end{array}$ & & \\
\hline $\mathrm{Bm}$ & $\begin{array}{l}\text { Hexacontium } \\
\text { palaeocenicum }\end{array}$ & $\begin{array}{l}\text { Below } \\
7-1 \quad P \\
\text { (Below }\end{array}$ & & &  & & \\
\hline $\mathrm{Bm}$ & $\begin{array}{l}\text { Entapium } \\
\text { regulare }\end{array}$ & $\begin{array}{l}\text { Below } \\
7-1 \quad \text { P } \\
\text { (Below }\end{array}$ & $\begin{array}{l}\text { Below } \\
43-1 \quad \text { G } \\
\text { (Below } 382 \text { ) }\end{array}$ & & $\begin{array}{l}\text { Below } \\
7-4 \\
\text { (Below 215) }\end{array}$ & & \\
\hline $\mathrm{Bm}$ & $\begin{array}{l}\text { Bekoma } \\
\text { campechensis }\end{array}$ & & & & $\begin{array}{l}\text { Below } \\
7-4 \quad \quad P \\
\text { (Below } 215 \text { ) }\end{array}$ & & \\
\hline $\mathrm{Bm}$ & $\begin{array}{l}\text { Clathrocycloma } \\
\text { parcum }\end{array}$ & & & & $\begin{array}{l}\text { Below } \\
7-4 \\
\text { (Below }\end{array}$ & & \\
\hline $\mathrm{Bm}$ & $\begin{array}{l}\text { Lamptonium } \\
\text { incohatum }\end{array}$ & $\begin{array}{l}\text { Below } \\
10-2 \quad P \\
\text { (Below 469) }\end{array}$ & & & ? & $\begin{array}{l}\text { Below } \\
11(\text { CC) M } \\
\text { (Below 604) }\end{array}$ & \\
\hline $\mathrm{Bm}$ & $\begin{array}{l}\text { Thecosphaerella } \\
\text { rotunda }\end{array}$ & $\begin{array}{l}\text { Below } \\
7-1 \quad P \\
\text { (Below } 441 \text { ) }\end{array}$ & $\begin{array}{l}\text { Below } \\
42 \text { (CC) M } \\
\text { (Below 376) }\end{array}$ & & $\begin{array}{l}\text { Below } \\
7(\mathrm{CC}) \quad \mathrm{M} \\
\text { (Below 216) }\end{array}$ & & \\
\hline $\mathrm{Bm}$ & $\begin{array}{l}\text { Amphisphaera } \\
\text { minor }\end{array}$ & $\begin{array}{l}\text { Below } \\
10-2 \quad M \\
\text { (Below 469) }\end{array}$ & & & $\begin{array}{l}\text { Below } \\
7 \text { (CC) M } \\
\text { (Below 216) }\end{array}$ & & \\
\hline $\mathrm{Bm}$ & $\begin{array}{l}\text { Heliostylus } \\
\text { sp (p) }\end{array}$ & $\begin{array}{l}\text { Below } \\
10-2 \quad M \\
\text { (Below } 469 \text { ) }\end{array}$ & $\begin{array}{l}42(\mathrm{CC}) \\
43-1 \quad P \\
(376-382)\end{array}$ & & $\begin{array}{l}\text { Below } \\
7(\text { CC) M } \\
\text { (Below 216) }\end{array}$ & & \\
\hline $\mathrm{Bm}$ & $\begin{array}{l}\text { Diploplegma } \\
\text { somphum }\end{array}$ & & & & $\begin{array}{l}\text { Below } \\
7(\mathrm{CC}) \quad \mathrm{P} \\
\text { (Below 216) }\end{array}$ & & \\
\hline $\mathrm{Bm}$ & $\begin{array}{l}\text { Pterocodon (?) } \\
\text { ampla (?) }\end{array}$ & $\begin{array}{l}\text { Below } \\
7-1 \quad M \\
\text { (Below } 441 \text { ) }\end{array}$ & & & $\begin{array}{l}\text { Within } \\
8-1 \\
(220)\end{array}$ & & \\
\hline $\mathrm{Bm}$ & $\begin{array}{l}\text { Clathrocycloma } \\
\text { capitaneum }\end{array}$ & $\begin{array}{l}\text { Below } \\
10-2 \quad P \\
\text { (Below } 469 \text { ) }\end{array}$ & & & $\begin{array}{l}\text { Within } \\
8-1 \quad \text { M } \\
(220)\end{array}$ & & \\
\hline $\mathrm{Bm}$ & $\begin{array}{l}\text { Lamptonium } \\
\text { pennatum }\end{array}$ & $\begin{array}{l}\text { Below } \\
10-2 \quad \text { G } \\
\text { (Below 469) }\end{array}$ & & & $\begin{array}{l}\text { Within } \\
8-1 \\
(220)\end{array}$ & & \\
\hline $\mathrm{Bm}$ & $\begin{array}{l}\text { Spongodiscus } \\
\text { americanus }\end{array}$ & $\begin{array}{l}\text { Below } \\
7-1 \quad M \\
\text { (Below } 441 \text { ) }\end{array}$ & $\begin{array}{l}\text { Below } \\
\text { 43-1 } \quad \text { P } \\
\text { (Below } 382 \text { ) }\end{array}$ & & $\begin{array}{l}\text { Below } \\
\begin{array}{l}8(\mathrm{CC}) \quad \mathrm{G} \\
\text { (Below 221) }\end{array}\end{array}$ & $\begin{array}{l}\text { Below } \\
10(C C) \quad P \\
\text { (Below 593) }\end{array}$ & \\
\hline $\mathrm{Bm}$ & $\begin{array}{l}\text { Phormocyrtis } \\
\text { striata exquisita }\end{array}$ & $\begin{array}{l}9-2 \\
10-2 \quad M \\
(460-469)\end{array}$ & & & $\begin{array}{l}\text { Below } \\
8(\mathrm{CC}) \quad \mathrm{G} \\
\text { (Below 221) }\end{array}$ & $\begin{array}{lr}\text { Below } & \\
11-2 & M \\
\text { (Below 602) }\end{array}$ & \\
\hline
\end{tabular}


TABLE 9 - Continued

\begin{tabular}{|c|c|c|c|c|c|c|c|}
\hline & Events & 146 & 149 & 151 & 152 & 153 & Radiolarian Zones \\
\hline $\mathrm{Bm}$ & $\begin{array}{l}\text { Buryella } \\
\text { pentadica }\end{array}$ & $\begin{array}{l}\text { Below } \\
9-2 \quad \text { P } \\
\text { (Below 460) }\end{array}$ & & & $\begin{array}{l}\text { Below } \\
8 \text { (CC) G } \\
\text { (Below 221) }\end{array}$ & $\begin{array}{l}\text { Below } \\
11(\text { CC) M } \\
\text { (Below 604) }\end{array}$ & \multirow{7}{*}{ Unzoned } \\
\hline $\mathrm{Bm}$ & $\begin{array}{l}\text { Bekoma (?) } \\
\text { demissa }\end{array}$ & $\begin{array}{l}10-2 \\
10(\mathrm{CC}) \quad \mathrm{P} \\
(469)\end{array}$ & & & $\begin{array}{l}\text { Below } \\
8(\text { CC) } \\
\text { (Below 221) }\end{array}$ & & \\
\hline $\mathrm{Bm}$ & $\begin{array}{l}\text { Stylotrochus } \\
\text { alveatus }\end{array}$ & $\begin{array}{l}\text { Below } \\
10-2 \quad \text { M } \\
\text { (Below 469) }\end{array}$ & & & $\begin{array}{l}\text { Below } \\
8 \text { (CC) G } \\
\text { (Below 221) }\end{array}$ & $\begin{array}{lr}\text { Below } \\
11-2 \quad M \\
\text { (Below } 602 \text { ) }\end{array}$ & \\
\hline $\mathrm{Bm}$ & $\begin{array}{l}\text { Stylosphaera } \\
\text { coronata coronata }\end{array}$ & $\begin{array}{l}\text { Below } \\
10-2 \quad \text { G } \\
\text { (Below 469) }\end{array}$ & $\begin{array}{l}\text { Below } \\
43-1 \quad \text { G } \\
\text { (Below } 382 \text { ) }\end{array}$ & & $\begin{array}{l}\text { Below } \\
8 \text { (CC) G } \\
\text { (Below 221) }\end{array}$ & $\begin{array}{l}\text { Below } \\
11-2 \quad \text { G } \\
\text { (Below 602) }\end{array}$ & \\
\hline $\mathrm{Bm}$ & $\begin{array}{l}\text { Stylosphaera } \\
\text { goruna }\end{array}$ & $\begin{array}{l}\text { Below } \\
10-2 \quad M \\
\text { (Below 469) }\end{array}$ & & & $\begin{array}{l}\text { Below } \\
8 \text { (CC) G } \\
\text { (Below 221) }\end{array}$ & $\begin{array}{lr}\text { Below } & \\
11-2 & M \\
\text { (Below 602) }\end{array}$ & \\
\hline $\mathrm{Bm}$ & $\begin{array}{l}\text { Axoprunum } \\
\text { pierinae group }\end{array}$ & $\begin{array}{l}\text { Below } \\
10-2 \quad M \\
\text { (Below } 469 \text { ) }\end{array}$ & & & $\begin{array}{l}\text { Below } \\
8 \text { (CC) M } \\
\text { (Below 221) }\end{array}$ & & \\
\hline $\mathrm{Bm}$ & $\begin{array}{l}\text { Buryella } \\
\text { tetradica }\end{array}$ & $\begin{array}{l}\text { Below } \\
10-2 \quad \text { G } \\
\text { (Below 469) }\end{array}$ & & & $\begin{array}{l}\text { Below } \\
8 \text { (CC) G } \\
\text { (Below 221) }\end{array}$ & $\begin{array}{l}\text { Below } \\
11 \text { (CC) M } \\
\text { (Below 604) }\end{array}$ & \\
\hline
\end{tabular}

\section{Species List}

The purpose of this list is to provide bibliographic references to the taxa mentioned in this chapter. If the published literature contains several different concepts of the limits of a species, the reference cited conforms to the concept as applied here. The list also serves as an index, since it directs the reader to the references to each taxon within this chapter.

Acrobotrys spp. Riedel and Sanfilippo, 1971, p. 1601, pl. 1J, figs.

12-16; pl. 2J, figs. 13-15; pl. 3F, fig. 8 .

This chapter: Tab. 3,5 .

Amphicraspedum murrayanum Haeckel - Sanfilippo and Riedel, 1973.

This chapter: Tab. 2, 4, 6, 9; Site 150 list; Text-fig. 2.

Amphicraspedum prolixum group Sanfilippo and Riedel, 1973.

This chapter: Tab. 2, 4, 6, 9; Site 150 list; Text-fig. 2 .

Amphicraspedum prolixum Sanfilippo and Riedel, 1973.

This chapter: Tab. 2, 4, 6, 9; Site 150 list; Text-fig. 2.

Amphisphaera minor (Clark and Campbell) - Sanfilippo and Riedel,

1973.

This chapter: Tab. 2, 6, 9; Site 150 list.

Amphiternis clava (Ehrenberg) - Foreman, 1973.

This chapter: Tab. 2, 4 .

Amphiternis sp. cf. Stichomitra alamedaensis (Campbell and Clark)

- Foreman, 1973.

This chapter: Tab. 2; Site 150 list.

Amphymenium splendiarmatum Clark and Campbell, 1942, p. 46, pl. 1, figs. 12, 14 .

This chapter: Tab. 4, 9; Text-fig. 2.

Anthocyrtoma sp. Riedel and Sanfilippo, 1970, p. 524, pl. 6, figs. $2-4$.

This chapter: Pl. 3, fig. 5.

Artophormis barbadensis (Ehrenberg) - Riedel and Sanfilippo, 1970 , p. 532, pl. 13, fig. 5.

This chapter: Tab. 3, 7, 9; Text-fig. 2.

Artophormis dominasinensis (Ehrenberg) - Riedel and Sanfilippo, 1971 , p. 1592 , pl. 6 , fig. 6 .

This chapter: Tab. 3, 9; Text-fig. 2.

Artophormis gracilis Riedel, 1959, p. 300, pl. 2, figs. 12, 13.

This chapter: Tab. 1, 3, 5, 7, 9; Text-fig. 2 .

Astrosphaerin sp. A Sanfilippo and Riedel, 1973.

This chapter: Tab. 6 .
Astrosphaerin sp. B Sanfilippo and Riedel, 1973.

This chapter: Tab. 6.

Astrosphaerin sp. C Sanfilippo and Riedel, 1973.

This chapter: Tab. 6, 9; Text-fig. 2 .

Astrosphaerin sp. D. Sanfilippo and Riedel, 1973.

This chapter: Tab. 2, 6, 9; Text-fig. 2.

Astrosphaerin sp. E. Sanfilippo and Riedel, 1973.

This chapter: Tab. 2, 6, 9; Site 150 list; Text-fig. 2.

Astrosphaerin sp. F Sanfilippo and Riedel, 1973.

This chapter: Tab. 2, 6, 9; Text-fig. 2 .

Astrosphaerin (?) sp.

This chapter: Footnote to Tab. 6; Pl. 5, figs. 8, 9.

Axoprunum pierinae (Clark and Campbell) group - Sanfilippo and Riedel, 1973.

This chapter: Tab. 2, 6, 9; Site 150 list; Text-fig. 2.

Bekoma bidarfensis Riedel and Sanfilippo - Foreman, 1973.

This chapter: Tab. 2, 6 .

Bekoma campechensis Foreman, 1973.

This chapter: Tab. 2, 6, 9; Text-fig. 2.

Bekoma (?) demissa Foreman, 1973.

This chapter: Tab. 2, 6, 9; Text-fig. 2.

Bekoma divaricata Foreman, 1973.

This chapter: Tab. 2, 6, 9; Text-fig. 2.

Botryocyrtis spp. Riedel and Sanfilippo, 1971, p. 1602, pl. 1J, figs. $1-11$; pl. 2 J, figs. $10-12$; pl. $3 \mathrm{~F}$, fig. 7 .

This chapter: Tab. 3,5 .

Botryopyle dictyocephalus Haeckel group - Riedel and Sanfilippo, 1971 , p. 1602 , pl. 1 J, figs. $21-26$; pl. 2 J, figs. $16-18$; pl. $3 \mathrm{~F}$, figs. 9-12.

This chapter: Tab. 3, 5, 9; Text-fig. 2.

Botryopyle sp. A Riedel and Sanfilippo, 1971, p. 1602, pl. 2J, figs. 20,$21 ;$ pl. $3 \mathrm{~F}$, fig. 13.

This chapter: Tab. 3.

Buryella clinata Foreman, 1973.

This chapter: Tab. 2, 4, 6, 9; Site 150 list.

Buryella pentadica Foreman, 1973.

This chapter: Tab. 2, 6, 8, 9; Text-fig. 2.

Buryella tetradica Foreman, 1973.

This chapter: Tab. 2, 6 (with footnote), 8 (with footnote), 9;

Site 150 list; PI. 5, fig. 5; Text-fig. 2.

Calocyclas hispida (Ehrenberg) - Foreman, 1973.

This chapter: Tab. 2-7, 9; Text-fig. 2. 
Calocyclas turris Ehrenberg - (as Cycladophora turris) Riedel and Sanfilippo, 1970 , p. 529, pl. 13, figs. 3, 4.

This chapter: Tab. 3, 7, 9; Text-fig. 2.

Calocycletta costata (Riedel) - Riedel and Sanfilippo, 1970, p. 535, pl. 14, fig. 12 .

This chapter: Tab. 5, 9; Text-fig. 2.

Calocycletta robusta Moore, 1971, p. 743 , pl. 10, figs. 5, 6.

This chapter: Tab. 3, 5, 9; Text-fig. 2.

Calocycletta serrata Moore, 1972 , p. 148, pl. 2, figs. 1-3, This chapter: Footnote to Tab. 7.

Calocycletta virginis Haeckel - Riedel and Sanfilippo, 1970, p. 535, pl. 14 , fig. 10

This chapter: Tab. 3, 5, 7 (with footnote), 9; Text-fig. 2.

Calocycloma castum (Haeckel) - Foreman, 1973. This chapter: Site 150 list.

Cannartus mammifer (Haeckel) - Riedel, 1959, p. 291, pl. 1, fig. 4. This chapter: Tab. 3, 5; Text-fig. 2 .

Cannartus prismaticus (Haeckel) - Riedel and Sanfilippo, 1971, p. 1588 , pl. 2 C, figs. $11-13$; pl. 4 , fig. 5 .

This chapter: Tab. 1, 3, 5, 7, 9; Text-fig. 2.

Cannartus tubarius (Haeckel) - Riedel and Sanfilippo, 1971, p. 1588 , pl. $2 \mathrm{C}$, figs. 8-10.

This chapter: Tab. 1, 3, 5, 9; Text-fig. 2.

Cannartus violina Haeckel - Riedel, 1959, p. 290, pl. 1, fig. 3. This chapter: Tab. 3, 5, 9; Text-fig. 2 .

Carpocanistrum (?) azyx Sanfilippo and Riedel, 1973.

This chapter: Tab. 3 (with footnote), 4, 7, 9; Pl. 2, fig. 4, Text-fig. 2.

Carpocanopsis bramlettei Riedel and Sanfilippo, 1971, p. 1597, pl, 2G, figs. 8-14; pl. 8, fig. 7.

This chapter: Tab. 3, 5, 7, 9; Text-fig. 2.

Carpocanopsis cingulata Riedel and Sanfilippo, 1971, p. 1597, pl. 2G, figs. $17-21$; pl. 8 , fig. 8 .

This chapter: Tab. 1, 3, 5, 7, 9; Text-fig. 2 .

Carpocanopsis favosa (Haeckel) - Riedel and Sanfilippo, 1971, p. 1597 , pl. $2 \mathrm{G}$, figs. 15,16 ; pl. 8 , figs. 9-11.

This chapter: Tab. 5, 7, 9; Text-fig. 2 .

Carposphaera subbotinae (Borisenko) - Sanfilippo and Riedel, 1973.

This chapter: Tab. 6,9.

Centrobotrys gravida Moore, 1971 , p. 744 , pl. 5 , fig. 8 . This chapter: Tab. 3, 5, 7 .

Centrobotrys petrushevskayae Sanfilippo and Riedel, 1973. This chapter: Tab. 3, 5, 7, 9 .

Centrobotrys thermophila Petrushevskaya - Nigrini, 1967, p. 49, text-fig. 26, pl. 5, fig. 7 .

This chapter: Tab. 3, 5, 7, 9; Text-fig. 2 .

Ceratospyris articulata Ehrenberg - Sanfilippo and Riedel, 1973. This chapter: Tab. 4, 9; Text-fig. 2.

Clathrocycloma capitaneum Foreman, 1973.

This chapter: Tab. 2, 6, 9; Text-fig. 2.

Clathrocycloma parcum Foreman, 1973.

This chapter: Tab. 4, 6, 9; Text-fig. 2.

Coccodiscid cf. Lithocyclia aristotelis group. This chapter: Pl. 2, figs. 5, 6 .

Cryptoprora sp. Sanfilippo and Riedel, 1973, pl. 35, fig. 5. This chapter: Footnote to Tab. 3.

Cyclampterium (?) leptetrum Sanfilippo and Riedel, 1970, p. 456 , pl. 2, figs. 11, 12 .

This chapter: Tab. 1, 5, 7, 9; Text-fig. 2 .

Cyclampterium (?) milowi Riedel and Sanfilippo - Sanfilippo and Riedel in Sanfilippo et al., in press. This chapter: Tab. 1, 3, 5, 7, 9; Text-fig. 2 .

Cyclampterium (?) pegetrum Sanfilippo and Riedel - Sanfilippo and Riedel in Sanfilippo et al., in press.

This chapter: Tab. 1, 3, 5, 7, 9; Text-fig. 2 .

Cyclampterium (?) tanythorax Sanfilippo and Riedel, 1970, p. 457, pl. 2, figs. 13,14 .

This chapter: Tab. 5 .

Cyrtocapsella cornuta Haeckel - Sanfilippo and Riedel, 1970, p. 453 , pl. 1 , figs. $19,20$.

This chapter: Tab. 3, 5, 7, 9; Text-fig. 2 .

Cyrtocapsella elongata (Nakaseko) - Sanfilippo and Riedel, 1970, p. 452 , pl. 1 , figs. 11,12 .

This chapter: Tab. 3, 5 .
Cyrtocapsella japonica (Nakaseko) - Sanfilippo and Riedel, 1970, p. 452, pl. 1, figs. 13-15.

This chapter: Tab. 3.

Cyrtocapsella tetrapera Haeckel - Sanfilippo and Riedel, 1970, p. 453 , pl. 1, figs. 16-18.

This chapter: Tab. 1, 3, 5, 7, 9; Text-fig. 2.

Dendrospyris fragoides Sanfilippo and Riedel, 1973. This chapter: Tab. 2, 6, 9; Site 150 list.

Dictyophimus craticula Ehrenberg - Sanfilippo and Riedel, 1973. This chapter: Tab. 3, 4, 9; Text-fig. 2 .

Dictyospyris discus Sanfilippo and Riedel, 1973. This chapter: Tab. 2, 6, 9; Site 150 list; Text-fig. 2.

Dictyospyris melissium Sanfilippo and Riedel, 1973. This chapter: Tab. 4, 9; Text-fig. 2.

Diploplegma somphum Sanfilippo and Riedel, 1973. This chapter: Tab. 6, 9 .

Dorcadospyris alata (Riedel) - Riedel and Sanfilippo, 1970, p. 523, pl. 14 , fig. 5 .

This chapter: Tab. 5, 9; Text-fig. 2 .

Dorcadospyris ateuchus (Ehrenberg) - Riedel and Sanfilippo, 1971, p. 1590 , pl. $2 \mathrm{D}$, fig. 6 ; pl. $3 \mathrm{~A}$, figs. 9,10 .

This chapter: Tab. 1, 3, 5, 7, 9; Text-fig. 2 .

Dorcadospyris circulus (Haeckel) - Moore, 1971, p. 739, pl. 8, figs. 3-5.

This chapter: Tab. 3 (with footnotes), 5, 7, 9; Pl. 1, fig. 8; Text-fig. 2.

Dorcadospyris confluens (Ehrenberg) - Sanfilippo and Riedel, 1973.

This chapter: Tab. 6, 9 .

Dorcadospyris dentata Haeckel - Riedel, 1957, p. 79, pl. 1, fig. 4. This chapter: Tab. 3, 5 .

Dorcadospyris forcipata (Haeckel) - Riedel and Sanfilippo, 1970, p. 523 , pl. 15 , fig. 7.

This chapter: Tab. 1, 3, 5, 7, 9; Text-fig. 2 .

Dorcadospyris papilio (Riedel) - Riedel and Sanfilippo, 1970, p. 523 , pl. 15 , fig. 5 .

This chapter: Tab. 1 (with footnotes), 3, 5, 7, 9; Text-fig. 2.

Dorcadospyris praeforcipata Moore, 1971 , p. 738 , pl. 9, figs. 4-7. This chapter: Tab. 1, 3, 5, 9; Text-fig. 2.

Dorcadospyris pseudopapilio Moore, 1971, p. 738, pl. 6, figs. 7, 8 . This chapter: Tab. 5,7 .

Dorcadospyris spinosa Moore, 1971, p. 739, pl. 7, figs. 1, 2. This chapter: Tab. 3 (with footnote), 7, 9; Pl. 2, fig. 2; Text-fig. 2 .

Entapium regulare Sanfilippo and Riedel, 1973. This chapter: Tab. 2, 3, 4, 6, 9; Text-fig. 2.

Eusyringium fistuligerum (Ehrenberg) - Foreman, 1973. This chapter: Tab. 3, 4, 7, 9; Text-fig. 2 .

Eusyringium lagena (Ehrenberg) - Foreman, 1973. This chapter: Tab. 4, 9; Text-fig. 2.

Giraffospyris lata Goll - Sanfilippo and Riedel, 1973. This chapter: Tab. 2, 6, 9; Site 150 list; Text-fig. 2.

Heliostylus sp(p). Sanfilippo and Riedel, 1973. This chapter: Tab. 2, 3 (with footnote), 4, 6, 9; Site 150 list; Pl. 2, fig. 3; Text-fig. 2.

Hexacontium palaeocenicum Sanfilippo and Riedel, 1973. This chapter: Tab. 2, 6, 9; Text-fig. 2.

Lamptonium (?) colymbus Foreman, 1973. This chapter: Tab. 2 .

Lamptonium (?) fabaeforme (?) chaunothorax Riedel and Sanfilippo, 1970 , p. 524 , pl. 5 , figs. 8,9 . This chapter: Tab. 4, 9; Site 150 list; Text-fig. 2.

Lamptonium fabaeforme (?) constrictum Riedel and Sanfilippo Foreman, 1973.

This chapter: Tab. 4, 9; Text-fig. 2.

Lamptonium fabaeforme fabaeforme (Krasheninnikov) (?) - Foreman, 1973.

This chapter: Tab. 2, 4, 6, 9; Site 150 list; Text-fig. 2.

Lamptonium (?) incohatum Foreman, 1973. This chapter: Tab. 2, 6 (with footnote), 8, 9; cf. pl. 5, fig. 10; Text-fig. 2.

Lamptonium pennatum Foreman, 1973. This chapter: Tab. 2, 6, 9; Site 150 list; Text-fig. 2.

Lamptonium sanfilippoae Foreman, 1973. This chapter: Tab. 6. 
Liriospyris parkerae Riedel and Sanfilippo, 1971, p. 1590, pl. 2C, fig. 15 ; pl. 5 , fig. 4 .

This chapter: Tab. 5, 9; Text-fig. 2.

Liriospyris stauropora (Haeckel) - Goll, 1968, p. 1431, pl. 175, figs. 1-3, 7; text-fig. 9.

This chapter: Tab. 5 .

Lithapium anoectum Riedel and Sanfilippo - Sanfilippo and Riedel, 1973.

This chapter: Tab. 4, 9; Text-fig. 2 .

Lithapium mitra (Ehrenberg) (?) - Riedel and Sanfilippo, 1970, p. 520 , pl. 4 , figs. 6,7 .

This chapter: Tab. 3, 4, 9; Text-fig. 2 .

Lithapium plegmacantha Riedel and Sanfilippo - Sanfilippo and Riedel, 1973.

This chapter: Tab. 3, 4, 9; Text-fig. 2 .

Lithelius foremanae Sanfilippo and Riedel, 1973. This chapter: Tab. 2, 6, 9; Text-fig. 2 .

Lithelius hexaxyphophorus (Clark and Campbell) - Sanfilippo and Riedel, 1973.

This chapter: Tab. 3, 4 (with footnote), 6, 9; PI. 3, fig. 6 .

Lithochytris archaea Riedel and Sanfilippo - Foreman, 1973. This chapter: Tab. 4, 6, 9; Site 150 list; P1. 5, fig. 1; Text-fig. 2.

Lithochytris vespertilio Ehrenberg - Riedel and Sanfilippo, 1970, p. 528 , pl. 9, figs. 8,9 .

This chapter: Tab. 3, 4, 6, 9; Text-fig. 2 .

Lithocyclia angusta (Riedel) - Riedel and Sanfilippo, 1970, p. 522, pl. 13 , figs. 1,2 .

This chapter: Tab. 1, 3, 5, 7, 9; Text-fig. 2 .

Lithocyclia aristotelis (Ehrenberg) group - Riedel and Sanfilippo, 1970, p. 522.

This chapter: Tab. 1, 3 (with footnote), 4, 7, 9; Text-fig. 2.

Lithocyclia crux Moore, 1971, p. 737, pl. 6, fig. 4. This chapter: Tab. 3, 7, 9; Text-fig. 2 .

Lithocyclia ocellus Ehrenberg group - Riedel and Sanfilippo, 1970, p. 522 , pl. 5 , figs. 1,2 .

This chapter: Tab. 1, 3, 4, 5, 9; Pl. 2, figs. 7, 8; Text-fig. 2.

Lithomitra docilis Foreman, 1973. This chapter: Tab. 3, 4, 6, 9; Text-fig. 2 .

Lithopera renzae Sanfilippo and Riedel, 1970, p. 454, pl. 1, figs. 21-23, 27.

This chapter: Tab. 5

Lophocyrtis biaurita (Ehrenberg) - Foreman, 1973.

This chapter: Tab. 4, 9; Text-fig. 2 .

Lophocyrtis (?) jacchia (Ehrenberg) - Riedel and Sanfilippo, 1971, p. 1594 , pl. 3C, figs. 4,5 ; pl. 7, fig. 16.

This chapter: Tab. 3,9 .

Lychnocanoma amphitrite Foreman, 1973.

This chapter: Tab. 3, 7, 9; Text-fig. 2.

Lychnocanoma anacolum Foreman, 1973. This chapter: Tab. 2

Lychnocanoma auxilla Foreman, 1973.

This chapter: Tab. 2, 4, 6, 8, 9; Text-fig. 2 .

Lychnocanoma babylonis (Clark and Campbell) group - Foreman, 1973.

This chapter: Tab. 2, 3, 4, 6, 8, 9; Site 150 list; Text-fig. 2.

Lychnocanoma bellum (Clark and Campbell) - Foreman, 1973. This chapter: Tab. 3, 4, 9; Text-fig. 2.

Lychnocanoma sp. aff. L. bellum (Campbell and Clark) - Foreman, 1973.

This chapter: Tab. $6,8,9$.

Lychnocanoma elongata (Vinassa) - Sanfilippo and Riedel in Sanfilippo et al., in press (=Lychnocanium bipes Riedel, 1959, p. 294 , pl. 2 , figs. 5,6 ).

This chapter: Tab. 1 (with footnote), 3, 5, 7, 9; Text-fig. 2.

Lychnocanoma sp. aff. L. elongata (Vinassa).

This chapter: Footnote to Tab. 1; Pl. 1, fig. 1.

Orbula comitata Foreman, 1973.

This chapter: Tab. 6; Text-fig. 2.

Orbula discipulus Foreman, 1973.

This chapter: Tab. 2.

Orbula ducalis Foreman, 1973.

This chapter: Tab. 2.

Periphaena decora Ehrenberg - Sanfilippo and Riedel, 1973.

This chapter: Tab. 3, 4, 6, 7, 9; Text-fig. 2.

Periphaena delta Sanfilippo and Riedel, 1973.

This chapter: Tab. 2, 4, 6, 9; Text-fig. 2.
Periphaena tripyramis triangula (Sutton) - Riedel and Sanfilippo, 1970 , p. 521 , pl. 4 , figs. $9,10$.

This chapter: Tab. 4, 9; Text-fig. 2.

Periphaena tripyramis tripyramis (Haeckel) - Sanfilippo and Riedel, 1973.

This chapter: Tab. 4, 9; Text-fig. 2.

Phormocyrtis cubensis (Riedel and Sanfilippo) - Foreman, 1973. This chapter: Tab. 2, 6, 9; Site 150 list; Text-fig. 2.

Phormocyrtis striata exquisita (Kozlova) - Foreman, 1973. This chapter: Tab. 2, 6, 8, 9; Site 150 list; Text-fig. 2.

Phormocyrtis striato striata Brandt-Foreman, 1973. This chapter: Tab. 2, 4, 6, 9; Site 150 list; Text-fig. 2.

Phormocyrtis turgida (Krasheninnikov) - Foreman, 1973. This chapter: Tab. 2, 6, 9; Site 150 list; Text-fig. 2.

Podocyrtis ampla Ehrenberg - Riedel and Sanfilippo, 1970, p. 533, pl. 12 , figs. 7,8 .

This chapter: Tab. 3, 4, 9; Text-fig. 2 .

Podocyrtis aphorma Riedel and Sanfilippo, 1970, p. 534, pl. 11, fig. 2.

This chapter: Tab. 4 (with footnote); cf. PI. 3, fig. 9.

Podocyrtis chalara Riedel and Sanfilippo, 1970, p. 535, pl. 12, figs. $2,3$.

This chapter: Tab. 2-4, 9; Text-fig. 2 .

Podocyrtis diamesa Riedel and Sanfilippo - Sanfilippo and Riedel, 1973.

This chapter: Tab. 4, 9; Text-fig. 2.

Podocyrtis dorus Sanfilippo and Riedel, 1973. This chapter: Tab. 4, 9; Text-fig. 2.

Podocyrtis goetheana (Haeckel) - Riedel and Sanfilippo, 1970, p. 535.

This chapter: Tab. 3, 4, 9; Text-fig. 2 .

Podocyrtis mitra Ehrenberg - Riedel and Sanfilippo, 1970, p. 534, pl. 11 , figs. 5,6 .

This chapter: Tab. 3, 4 (with footnote), 9; Pl. 3, fig. 1; Text-fig. 2.

Podocyrtis papalis Ehrenberg - Sanfilippo and Riedel, 1973.

This chapter: Tab. 2-6, 8, 9; Site 150 list; Text-fig. 2.

Podocyrtis phyxis Sanfilippo and Riedel, 1973.

This chapter: Tab. 4, 9; Text-fig. 2.

Podocyrtis platypus Sanfilippo and Riedel, 1973.

This chapter: Tab. 4, 9; Text-fig. 2.

Podocyrtis sinuosa Ehrenberg ? - Riedel and Sanfilippo, 1970, p. 534 , pl. 11 , figs. 3,4 .

This chapter: Tab. 3-5, 9; Text-fig. 2 .

Podocyrtis trachodes Riedel and Sanfilippo, 1970, p. 535, pl. 11, fig. 7 ; pl. 12, fig. 1 . This chapter: Tab. 4, 9; Text-fig. 2.

Podocyrtis sp. A This chapter: Tab. 4 (with footnote), 9; Pl. 4, figs. 1-3; Text-fig.

Podocyrtis sp. B

This chapter: Tab. 4 (with footnote), 9; Pl. 4, figs. 4-6; Text-fig. 2.

Psychospyris grandis Riedel and Sanfilippo, 1971, p. 1591, pl. 6, figs. 3-5. This chapter: Tab. 5 .

Psychospyris intermedia Riedel and Sanfilippo, 1971, p. 1591, pl. 5, fig. 11, pl. 6, figs. 1,2 .

This chapter: Tab. 5.

Psychospyris parva Riedel and Sanfilippo, 1971, p. 1591, pl. 5, figs. 8-10.

This chapter: Tab. 5 .

Pterocodon (?) ampla (Brandt) (?) - Foreman, 1973. This chapter: Tab. 2, 6, 9; Text-fig. 2 .

Pterocodon (?) tenellus Foreman, 1973.

This chapter: Tab. 2, 6, 9; Site 150 list; Text-fig. 2.

Rhabdolithis ellida Sanfilippo and Riedel, 1973. This chapter: Tab. 6, 9; Text-fig. 2.

Rhabdolithis pipa Ehrenberg - Sanfilippo and Riedel, 1973. This chapter: Tab. 4 (with footnote), 6, 9; Site 150 list; Pl. 3, figs. 7, 8; Text-fig. 2 .

Rhopalocanium ornatum Ehrenberg - Foreman, 1973. This chapter: Tab. 3, 4, 9; Text-fig. 2.

Saturnalin. This chapter: Footnote to Tab. 2, Pl. 1, fig. 2. 
Sethochytris triconiscus Haeckel (?) - Riedel and Sanfilippo, 1970, p. 528 , pl. 9 , fig. 6 .

This chapter: Tab. 3, 4, 9; Text-fig. 2 .

Spongatractus balbis Sanfilippo and Riedel, 1973.

This chapter: Tab. 4, 9; Text-fig. 2.

Spongatractus pachystylus (Ehrenberg) - Sanfilippo and Riedel, 1973.

This chapter: Tab. 3, 4, 9; Text-fig. 2 .

Spongodiscus americanus Kozlova - Sanfilippo and Riedel, 1973. This chapter: Tab. 2, 4, 6, 8, 9 .

Spongodiscus cruciferus Clark and Campbell - Sanfilippo and Riedel, 1973.

This chapter: Tab. 2.

Spongodiscus phrix Sanfilippo and Riedel, 1973.

This chapter: Tab. 4, 9; Text-fig. 2.

Spongodiscus quartus bosoculus Sanfilippo and Riedel, 1973.

This chapter: Tab. 2, 4, 6, 8, 9; Text-fig. 2 .

Spongodiscus quartus quartus (Borisenko) - Sanfilippo and Riedel, 1973.

This chapter: Tab. 2, 6, 9; Site 150 list; Text-fig. 2 .

Spongodiscus rhabdostylus (Ehrenberg) - Sanfilippo and Riedel, 1973.

This chapter: Tab. 3, 4, 9; Text-fig. 2.

Spongomelissa adunca Sanfilippo and Riedel, 1973.

This chapter: Tab. 2, 6, 9; Text-fig. 2.

Stichocorys armata (Haeckel) - Sanfilippo and Riedel, in Sanfilippo et al., in press.

This chapter: Tab. 5 .

Stichocorys delmontensis (Campbell and Clark) - Sanfilippo and Riedel, 1970, p. 451 , pl. 1, fig. 9.

This chapter: Tab. 3, 5, 7, 9; Text-fig. 2.

Stichocorys diploconus (Haeckel) - Sanfilippo and Riedel, 1970, p. 451 , pl. 1, figs. 31,32 .

This chapter: Tab. 5 .

Stichocorys wolffii Haeckel - Riedel, 1957, p. 92, pl. 4, figs. 6, 7 . This chapter: Tab. 5, 9; Text-fig. 2 .

Stylosphaera coronata coronata Ehrenberg - Sanfilippo and Riedel, 1973.

This chapter: Tab. 2, 3, 4, 6, 8, 9; Site 150 list.

Stylosphaera coronata laevis Ehrenberg - Sanfilippo and Riedel, 1973.

This chapter: Tab. $3,4,6,9$.

Stylosphaera coronata sabaca Sanfilippo and Riedel, 1973.

This chapter: Tab. 2, 4, 6, 9; Text-fig. 2.

Stylosphaera goruna Sanfilippo and Riedel, 1973.

This chapter: Tab. 2, 4, 6, 8, 9; Text-fig. 2 .

Stylotrochus alveatus Sanfilippo and Riedel, 1973.

This chapter: Tab. 2, 6, 8, 9; Text-fig. 2 .

Stylotrochus nitidus Sanfilippo and Riedel, 1973.

This chapter: Tab. 2, 6, 9; Site 150 list; Text-fig. 2.

Tepka perforata Sanfilippo and Riedel, in Sanfilippo et al., in press. This chapter: Tab. 1, 3, 5 (with footnote), 7, 9; Text-fig. 2.

Tepka sp.

This chapter: Footnote to Tab. 5; Pl. 5, figs. $2,3$.

Thecosphaera larnacium Sanfilippo and Riedel, 1973.

This chapter: Tab. 2, 6, 9; Site 150 list; Text-fig. 2.

Thecosphaerella sp. cf. T. agdaraensis (Mamedov) - Sanfilippo and Riedel, 1973.

This chapter: Tab. 2.

Thecosphaerella ptomatus Sanfilippo and Riedel, 1973. This chapter: Tab. 3, 4, 6, 9; Site 150 list; Text-fig. 2 .

Thecosphaerella rotunda (Borisenko) - Sanfilippo and Riedel, 1973.

This chapter: Tab. 2, 3, 4, 6, 9; Site 150 list.

Theocampe amphora (Haeckel) group - Foreman, 1973. This chapter: Tab. 3, 4, 9; Text-fig. 2.

Theocampe armadillo (Ehrenberg) group - Riedel and Sanfilippo, 1971 , p. 1601, pl. 3E, figs. 3-6.

This chapter: Tab. 3, 4, 5, 7, 9; Text-fig. 2 .

Theocampe mongolfieri (Ehrenberg) - Foreman, 1973. This chapter: Tab. 1-5, 7, 9; Text-fig. 2.

Theocampe pirum (Ehrenberg) - Riedel and Sanfilippo, 1971, p. 1601 , pl. 3E, figs. $10,11$.

This chapter: Tab. 3, 5, 7, 9; Text-fig. 2.

Theocampe urceolus (Haeckel) - Foreman, 1973.

This chapter: Tab. 3, 4, 9; Text-fig. 2 .
Theocorys acroria Foreman, 1973.

This chapter: Tab. 2, 4, 6, 9; Text-fig. 2.

Theocorys anaclasta Riedel and Sanfilippo - Foreman, 1973. This chapter: Tab. 4, 9; Text-fig. 2.

Theocorys anapographa Riedel and Sanfilippo - Foreman, 1973. This chapter: Tab. 3 (with footnote), 4 (with footnote), 9; Pl. 3, figs. 10, 11; Text-fig. 2 .

Theocorys (?) phyzella Foreman, 1973.

This chapter: Tab. 2, 6 (with footnote), 9; Site 150 list; Text-fig. 2.

Theocorys (?) sp. cf. $T$. (?) phyzella

This chapter: Footnote to Tab. 6; Pl. 5, fig. 4.

Theocorys spongoconum Kling - Foreman, 1973.

This chapter: Tab. 1, 3 (with footnote), 5, 7, 9; Pl. 2, fig. 1; Text-fig. 2.

Theocotyle alpha Foreman, 1973.

This chapter: Tab. 4; Site 150 list.

Theocotyle auctor Foreman, 1973.

This chapter: Tab. 4, 6, 9; Text-fig. 2 .

Theocotyle cryptocephala (?) conica Foreman, 1973. This chapter: Tab. 4, 9; Text-fig. 2.

Theocotyle cryptocephala cryptocephala (Ehrenberg) (?) - Foreman, 1973.

This chapter: Tab. 4, 9; Text-fig. 2.

Theocotyle cryptocephala (?) nigriniae Riedel and Sanfilippo Foreman, 1973 .

This chapter: Tab. 4, 9; Text-fig. 2.

Theocotyle ficus (Ehrenberg) - Foreman, 1973.

This chapter: Tab. 2-4, 9; Text-fig. 2.

Theocotyle venezuelensis Riedel and Sanfilippo, 1970, p. 525, pl. 6, figs. 9,10 ; pl. 7, figs. 1,2 .

This chapter: Tab. 4, 9; Text-fig. 2.

Theocyrtis annosa (Riedel) - Riedel and Sanfilippo, 1970, p. 535, pl. 15 , fig. 9.

This chapter: Tab. 1, 3 (with footnote), 5, 7, 9; Text-fig. 2.

Theocyrtis tuberosa Riedel, 1959, p. 298, pl. 2, figs. 10, 11 .

This chapter: Tab. 1, 3 (with footnote), 5, 7, 9; Text-fig. 2.

Thyrsocyrtis bromia Ehrenberg - Riedel and Sanfilippo, 1971, p. 1596, pl. 8, fig. 6 .

This chapter: Tab. 3, 7, 9; Text-fig. 2.

Thyrsocyrtis hirsuta hirsuta (Krasheninnikov) - Foreman, 1973. This chapter: Tab. 4; Site 150 list.

Thyrsocyrtis hirsuta robusta Riedel and Sanfilippo - Foreman, 1973.

This chapter: Tab. 4, 9; Text-fig. 2.

Thyrsocyrtis hirsuta tensa - Foreman, 1973.

This chapter: Tab. 4, 9; Site 150 list; Text-fig. 2.

Thyrsocyrtis rhizodon Ehrenberg - Foreman, 1973.

This chapter: Tab. 1, 3, 4, 6 (with footnote), 9; Pl. 5, figs. 6, 7; Text-fig. 2.

Thyrsocyrtis sp. aff. T. rhizodon.

This chapter: Footnote to Tab. 6; Pl. 5, figs. 6, 7.

Thyrsocyrtis tarsipes Foreman, 1973.

This chapter: Tab. 2, 4, 6, 9; Text-fig. 2.

Thyrsocyrtis tetracantha (Ehrenberg) - Riedel and Sanfilippo, 1971, p. 1596.

This chapter: Tab. 3, 7, 9; Text-fig. 2.

Thyrsocyrtis triacantha (Ehrenberg) - Foreman, 1973. This chapter: Tab. 3-5, 9; Text-fig. 2.

Tristylospyris triceros (Ehrenberg) - Haeckel, 1887, p. 1033. This chapter: Tab. 1, 3, 5, 7, 9; Text-fig. 2.

Xiphospira circularis (Clark and Campbell) - Sanfilippo and Riedel, 1973.

This chapter: Tab. 2, 6, 9; Text-fig. 2.

\section{ACKNOWLEDGMENTS}

Financial support for this study was provided partly by NSF Grant No. GA-31284X and partly by the University of California. The onerous tasks of typing and proofreading were competently performed by M. A. Hanger and M. A. Neely. For access to samples from a core collected by Woods Hole Oceanographic Institution we are indebted to C. D. Hollister and Peter Lonsdale. 


\section{REFERENCES ${ }^{1}$}

Clark, B. L. and Campbell, A. S., 1942. Eocene radiolarian faunas from the Mt. Diablo area, California. Geol. Soc. Am., Spec. Paper 39, 112 p.

Foreman, H. P., 1973. Radiolaria of Leg 10 with systematics and ranges for the families Amphipyndacidae, Artostrobiidae, and Theoperidae. In Worzel, J. L., Bryant, W. et al., 1973. Initial Reports of the Deep Sea Drilling Project, Volume X. Washington (U. S. Government Printing Office).

Goll, R. M., 1968. Classification and phylogeny of Cenozoic Trissocyclidae (Radiolaria) in the Pacific and Caribbean Basins. Part I. J. Paleontol. 42(6), 1409.

Haeckel, E., 1887. Report on the Radiolaria collected by $\mathrm{H}$. M. S. Challenger during the years 1873-76. Rept. Voyage Challenger, Zool. 18. 1803 p.

Moore, T. C., Jr., 1971. Radiolaria. In Tracey, J. I., Jr., Sutton, G. H. et al., 1971. Initial Reports of the Deep Sea Drilling Project, Volume VIII. Washington (U. S. Government Printing Office). 727.

1972. Mid-Tertiary evolution of the radiolarian genus Calocycletta. Micropaleontology. 18(2), 144.

Nigrini, C., 1967. Radiolaria in pelagic sediments from the Indian and Atlantic Oceans. Bull. Scripps Inst. Oceanog. Univ. Calif. 11. 125 p.

\footnotetext{
${ }^{1}$ Note added in proof. Some items of information (complete pagination, plate numbers, etc.) have been deleted from these references by the editorial staff of the Deep Sea Drilling Project. Complete citations of some of the references, including the deleted items, may be found in the radiolarian chapters of Volumes VII and XIV of this series of Initial Reports.
}

Riedel, W. R., 1957. Radiolaria: a preliminary stratigraphy. Rept. Swedish Deep-Sea Expedit. 6 (3), 59. 1959. Oligocene and Lower Miocene Radiolaria in tropical Pacific sediments. Micropaleontology. 5 (3), 285.

Riedel, W. R. and Sanfilippo, A., 1970. Radiolaria, Leg 4, Deep Sea Drilling Project. In Bader, R. G., Gerard, R. D., et al., 1970. Initial Reports of the Deep Sea Drilling Project, Volume IV. Washington (U.S. Government Printing Office). 503.

, 1971. Cenozoic Radiolaria from the western tropical Pacific, Leg 7. In Winterer, E. L., Riedel, W. R. et al., 1971. Initial Reports of the Deep Sea Drilling Project, Volume VII. Washington (U. S. Government Printing Office, 1529.

Sanfilippo, A. and Riedel, W. R., 1970. Post-Eocene "closed" theoperid radiolarians. Micropaleontology. 16 (4), 446.

1973. Cenozoic Radiolaria (exclusive of theoperids, artostrobiids, and amphipyndacids) from the Gulf of Mexico, DSDP Leg 10. In Worzel, J. L., Bryant, W. et al., 1973. Initial Reports of the Deep Sea Drilling Project, Volume X. Washington (U. S. Government Printing Office).

Sanfilippo, A., Burckle, L. H., Martini, E. and Riedel, W. R., in press. Radiolarians, diatoms, silicoflagellates and calcareous nannofossils in the Mediterranean Neogene. Micropaleontology.

\section{PLATES}

In the explanations to the figures, the sample numbers and slide designations (in the form "Ph.2", "S1.1", "Cse.2", etc.) indicate preparations in our collection at Scripps Institution of Oceanography, and designations in the form "R45/1" indicate England Finder positions of the illustrated specimens on the slides. 


\section{PLATE 1}

Figure 1 Lychnocanoma sp., ancestral to L. elongata (see footnote $b$ to Table 1). 146-2-5 $(80-82 \mathrm{~cm}), \mathrm{Ph} .2$, $\mathrm{H} 29 / 4(255 \mathrm{X})$

Figure 2 Saturnalin gen. et. sp. indet. (see footnote a to Table 2). $146-7-1(83-86 \mathrm{~cm}), \mathrm{S} 1.3, \mathrm{D} 12 / 1$ (255X)

Figures 3,4 Radiolarians, gen. et sp. indet. (see footnote $b$ to Table 2)

3: $146-10-2(85-90 \mathrm{~cm}), \mathrm{S} 1.3 \mathrm{~A}, \mathrm{~L} 23 / 3(255 \mathrm{X})$

4: same slide, $\mathrm{P} 20 / 4$ (150X)

Figures 5,6 Pyritized radiolarians (see footnote $\mathrm{c}$ to Table 2)

5: $146-10-2(138-142 \mathrm{~cm}), \mathrm{S} 1.1, \mathrm{~F} 41 / 4(255 \mathrm{X})$

6: same slide, D27/3 (255X)

Figure 7 Dorcadospyris sp. (see footnote a to Table 3) $149-25 \cdot 2(28-30 \mathrm{~cm})$, Cse.2, U33/2 (150X)

Figure 8 Dorcadospyris circulus 149-26-2 (100-102 cm), Cse.2, $016 / 0(150 X)$

Figures 9, 10 Dorcadospyris $\mathrm{sp}(\mathrm{p})$. (see footnote b to Table 3)

9: $149-28(\mathrm{CC})$, Cse.2, R45/0 (150X)

10: 149-28(CC), Cse.1, Q30/1 (150X) 
PLATE 1
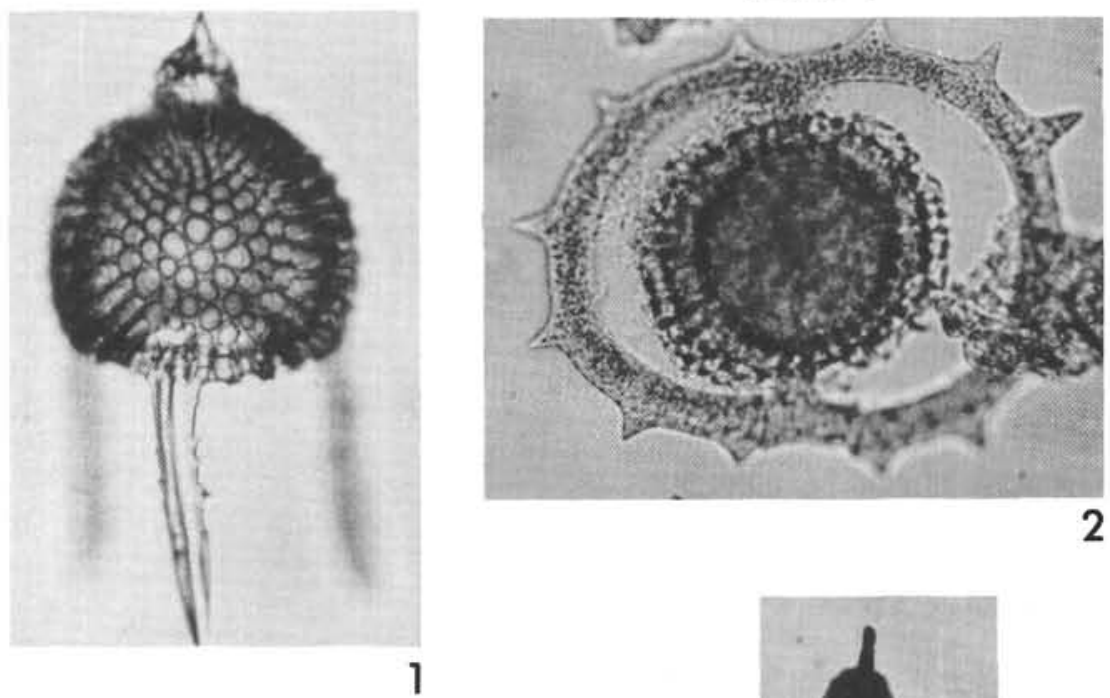

\section{1}

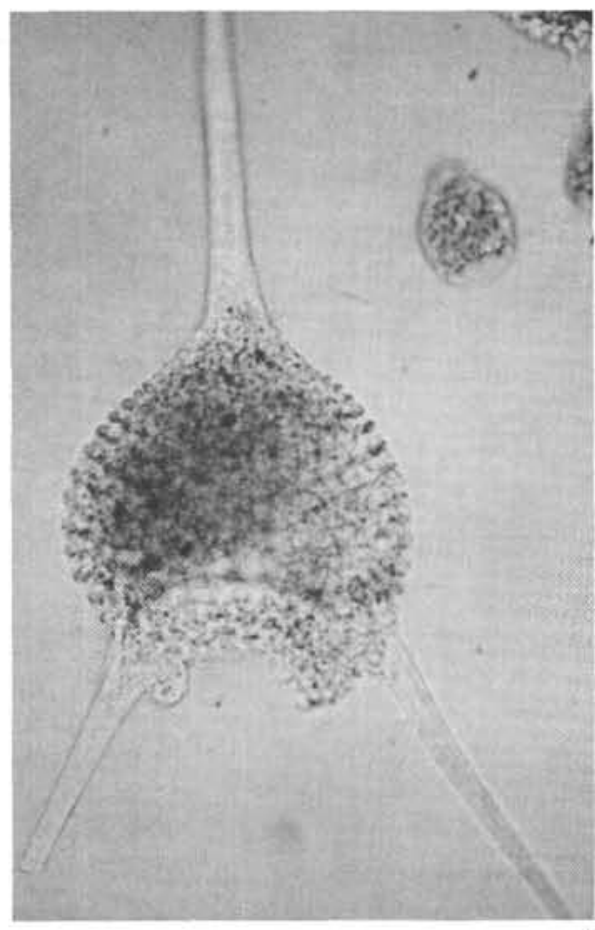

4

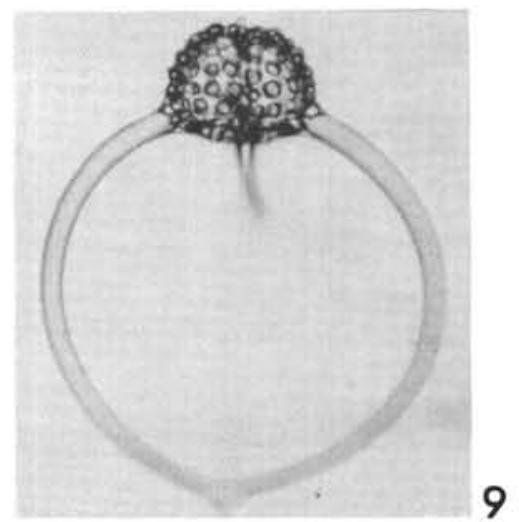

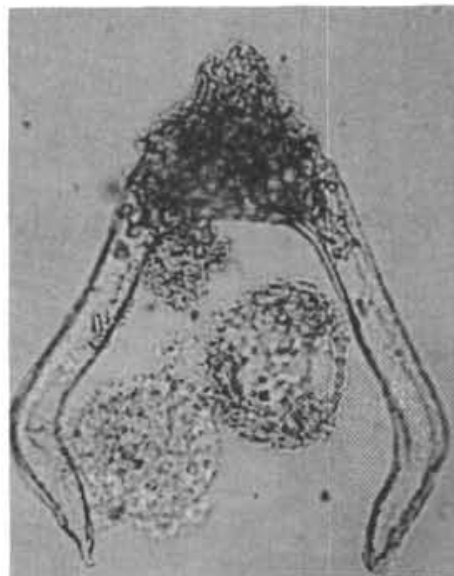

3

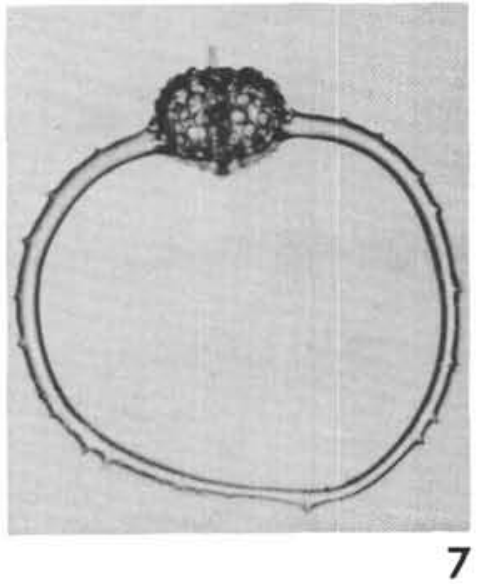

6
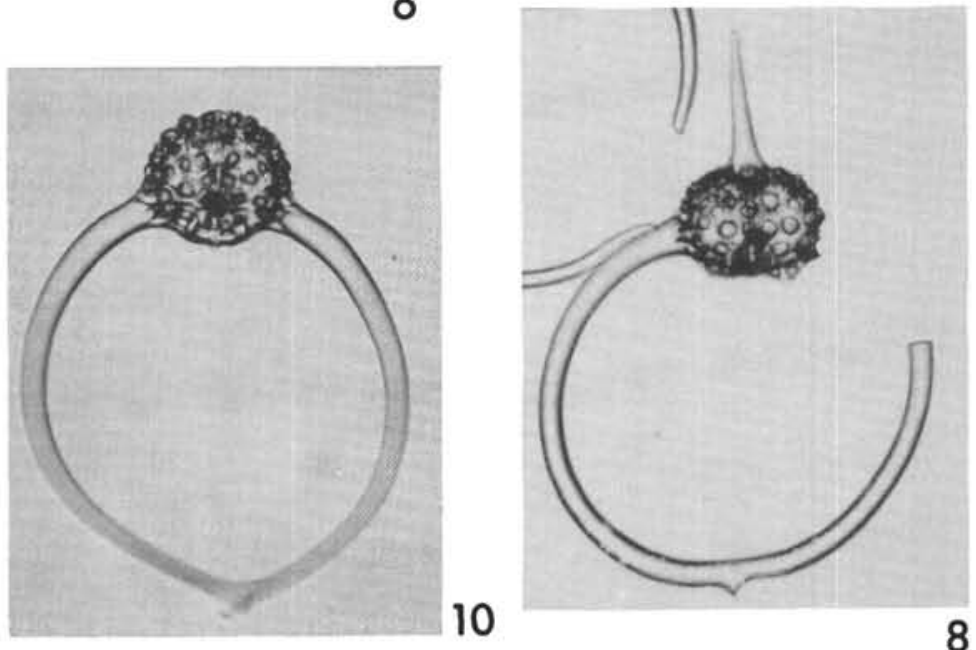


\section{PLATE 2}

Figure 1

Figure 2

Figure 3

Figure 4

Figures 5,
Theocorys spongoconum (see footnote c to Table 3 ) 149-29(CC), Ph.1, U42/3 (255X)

Dorcadospyris spinosa (see footnote d to Table 3) 149-30-2 (23-24 cm), Cse, X38/1 (150X)

Heliostylus sp. (see footnote e to Table 3) 149-30-1 $(87-89 \mathrm{~cm}), \mathrm{S} 1.1, \mathrm{~T} 44 / 0$ (150X)

Carpocanistrum azyx (see footnote $\mathrm{f}$ to Table 3) $149-32-3(38-42 \mathrm{~cm}), \mathrm{S} 1.2, \mathrm{E} 35 / 4(255 \mathrm{X})$

Coccodiscid gen. et sp. indet. with some similarity to Lithocyclia aristotelis group (see footnote i to Table 3)

5: 149-33(CC), Cse.1, D34/1 (150X)

6: same slide, $048 / 0$ (150X)

Figures 7, $8 \quad$ Lithocyclia ocellus group (see footnote i to Table 3) 7: 149-33(CC), Cse.1, N34/3 (150X)

8: same sample, Cse.2, D21/4 (150X) 
PLATE 2
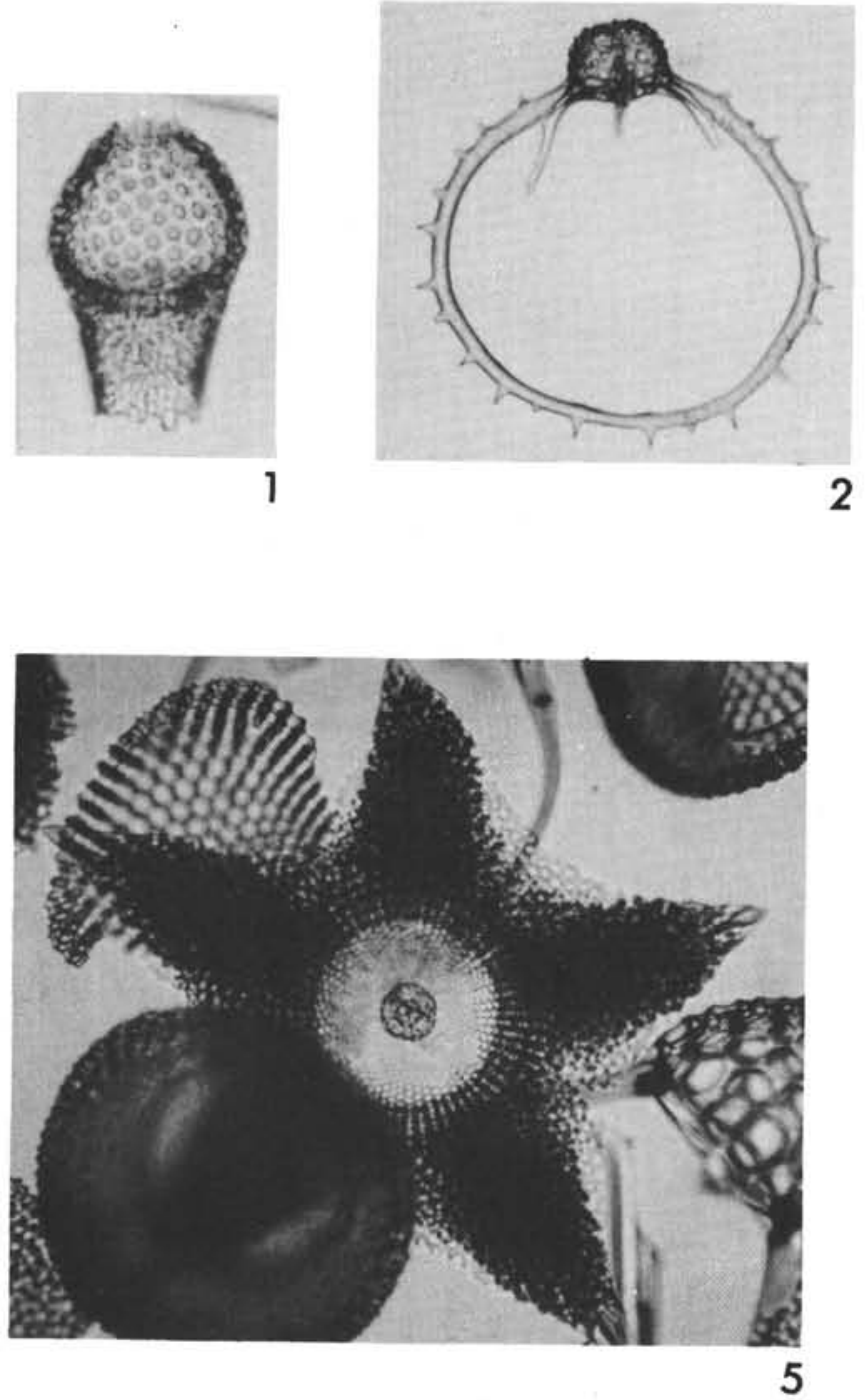

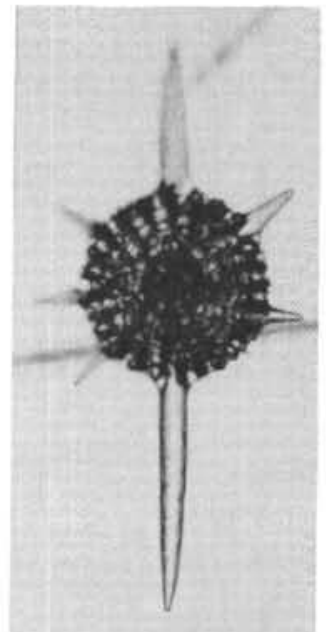

3

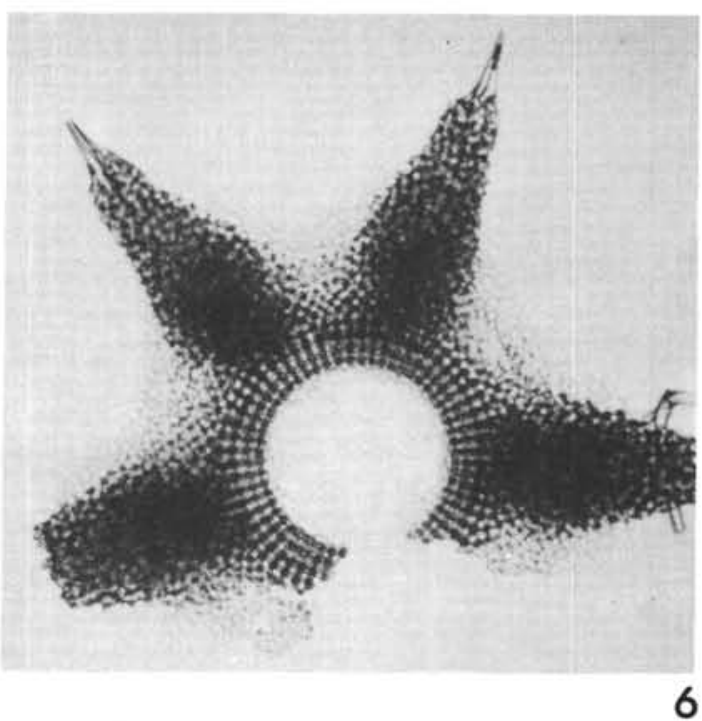

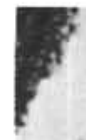

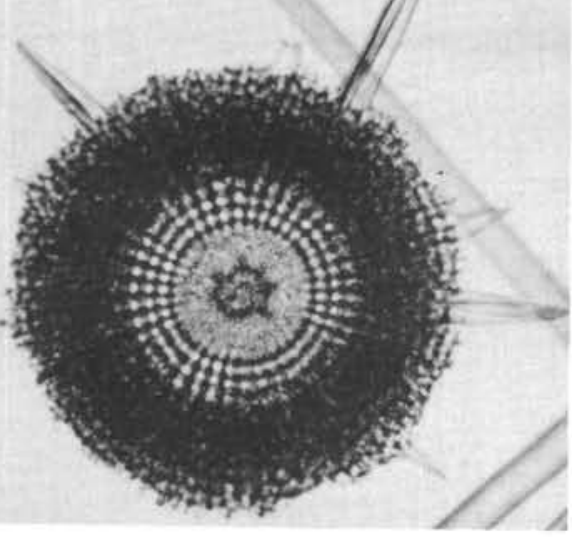

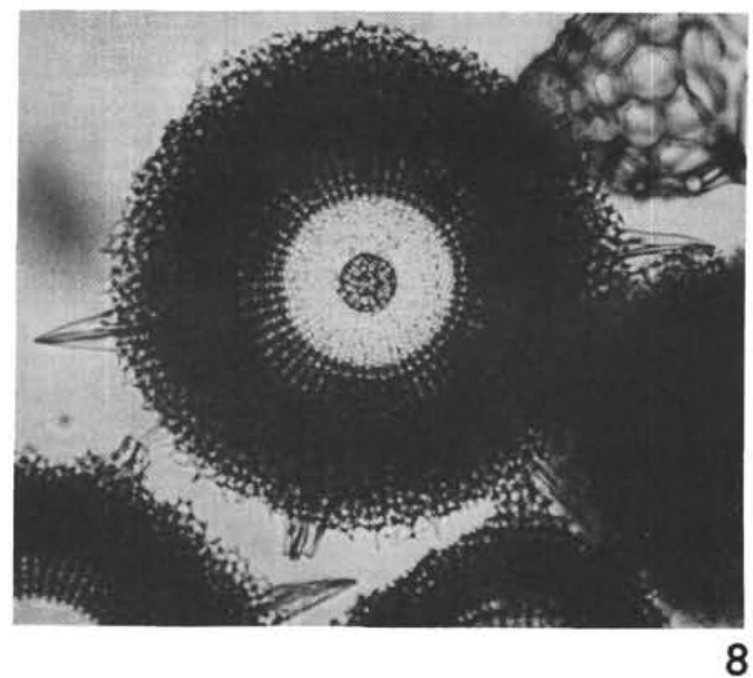




\section{PLATE 3}

Figure 1 A well-preserved specimen of Podocyrtis mitra showing delicately subdivided pores. 149-35-2 (123-125 $\mathrm{cm})$, Cse.1, J27/3 (255X)

Figure 2 Coccodiscid gen. et sp. indet., found only in the lower part of Core 149-41. 149-41(CC), Cse.1, D8/0 $(150 \times)$

Figures 3, 4 Many-rayed spongodiscids with pronounced marginal pylome, occurring commonly in Cores 149-32 through 149-34.

3: 149-34(CC), Cse.2, V41/3

4: 149-34(CC), Cse.2, C10/0 (95X)

Figure 5 Anthocyrtoma sp., resembling that illustrated by Riedel and Sanfilippo, 1970, Pl. 6, fig. 4, but showing a large tube-like or sack-like protuberance from the thoracic wall just above the lumbar stricture (right hand side of illustration). 149-38(CC), Cse.1, R41/4 $(150 X)$

Figure 6 Lithelius hexaxyphophorus (see footnote a to Table 4) $149-38(\mathrm{CC})$, Cse.1, N26/3 (150X)

Figures 7, 8 Rhabdolithis pipa (see footnote b to Table 4)

7: 149-34-2 (18-20 cm), Cse.1, L32/0 (150X)

8: Detail, showing swollen part of Rhabdolithis pipa. 149-34-2 (18-20 cm), Cse.1, U40/4 (480X)

Figure 9 Pterocorythid gen. et sp. indet., superficially similar to Podocyrtis aphorma (see footnote c to Table 4). 149-35-1 (104-106 cm), Cse.2, D40/0 (255X)

Figures 10,11 Theocorys anapographa (see footnote e to Table 4)

10: 149-32(CC), Ph.1, H15/1 (255X)

11: 149-32(CC), Ph.1, U41/2 (255X) 
PLATE 3
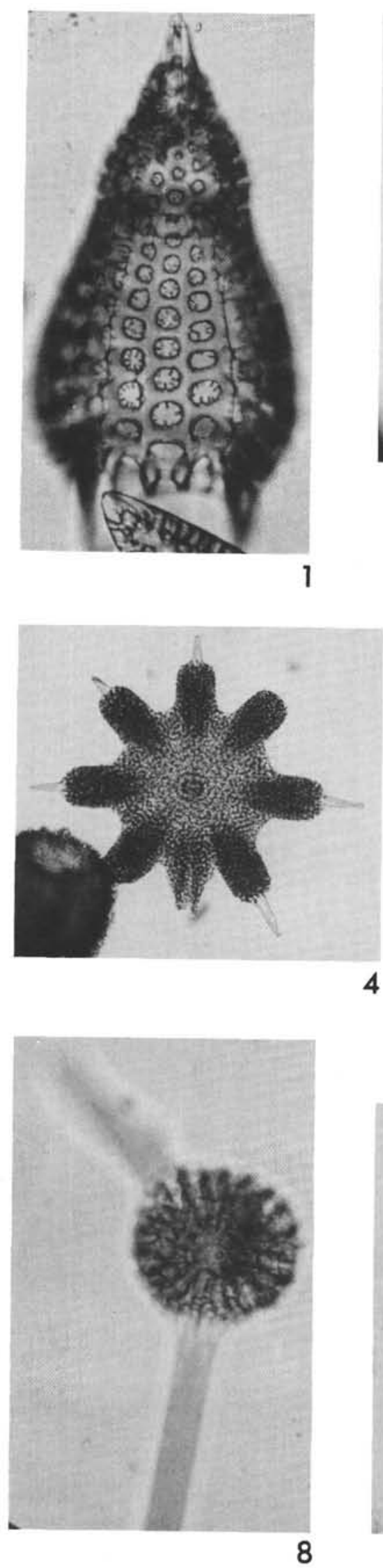

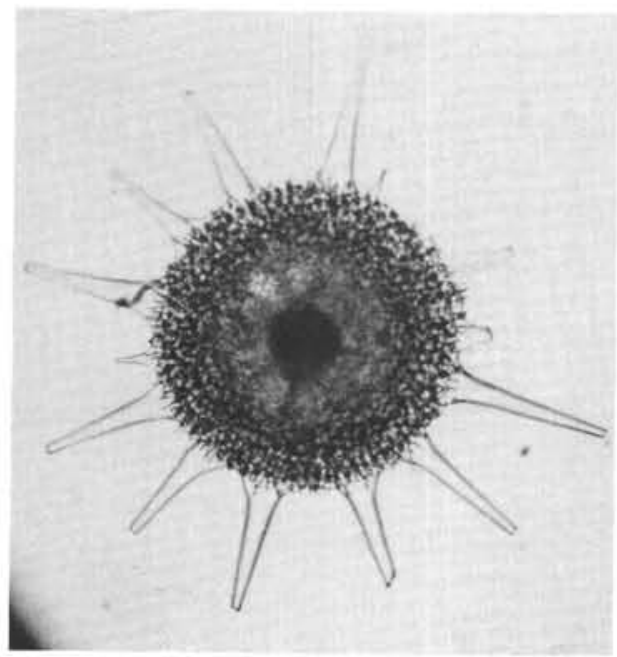

2

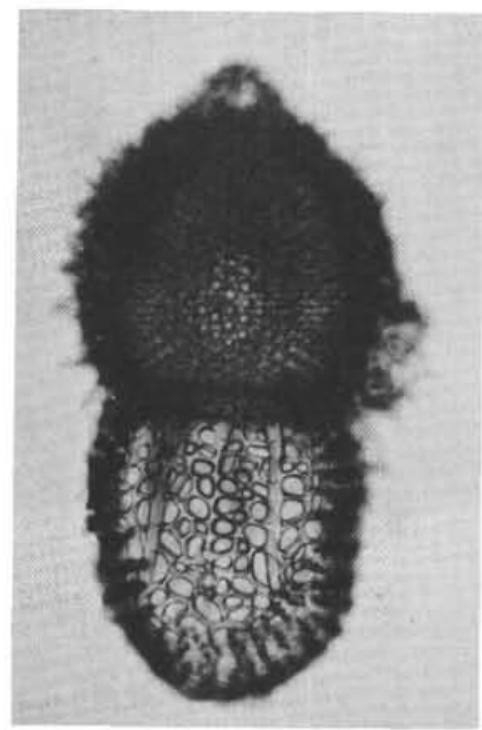

5

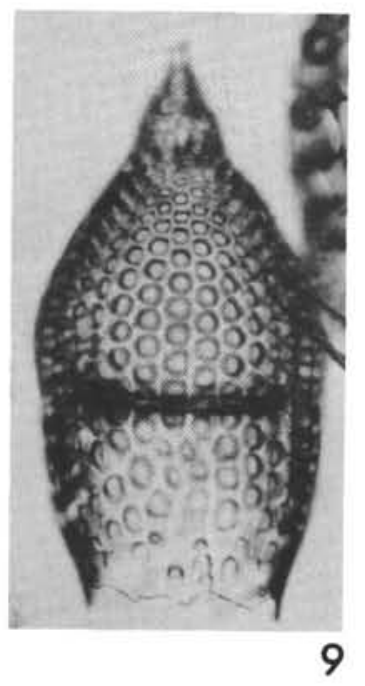

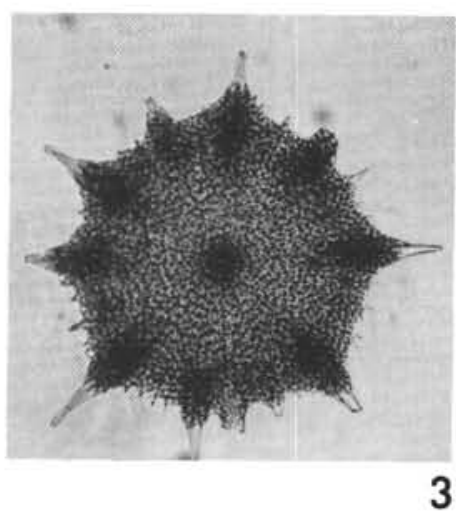
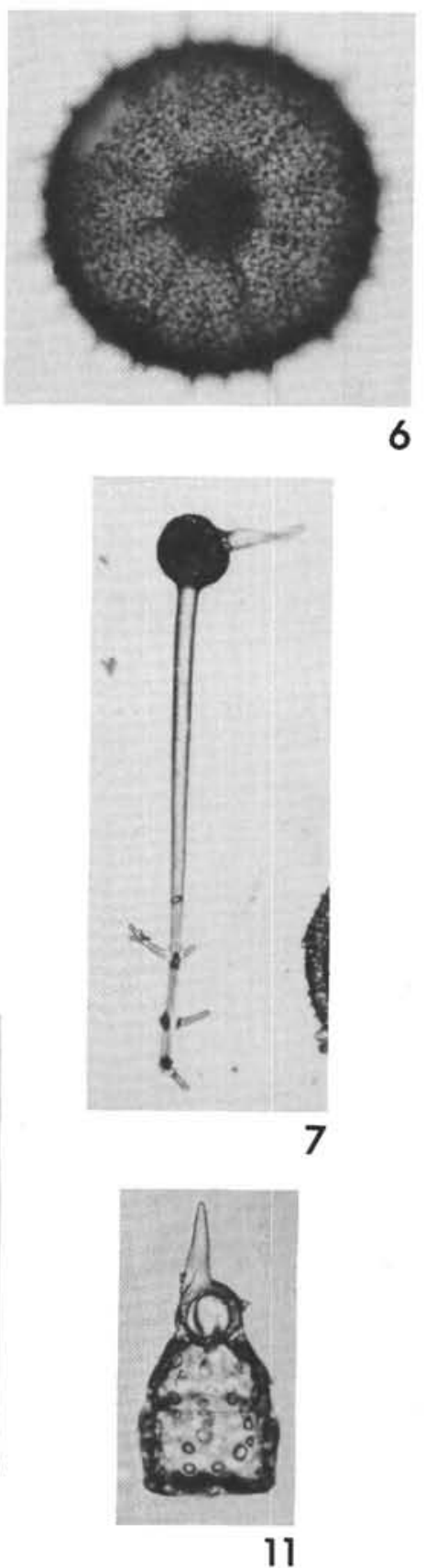


\section{PLATE 4}

Figures 1-3 Podocyrtis sp. A (see footnote $\mathrm{f}$ to Table 4) 1: $149-37-3(54-56 \mathrm{~cm})$, Cse.2, F50/1 (255X)

2. 149-37-3 (54-56 cm), Cse.2, M12/2 (255X)

3. 149-37-4 (10-12 cm), Cse.2, P19/4 (255X)

Figures 4-6 Podocyrtis sp. B (see footnote g to Table 4) 4: $149-37-3(54-56 \mathrm{~cm})$, Cse.2, Y45/4 (255X) 5: $149-37-4(10-12 \mathrm{~cm})$, Cse.1, S21/4 (255X) 6: $149-37-3(54-56 \mathrm{~cm})$, Cse.2, Q16/4 (255X)

Figures 7-12 Unidentified, apparently Cretaceous radiolarians 7: 146-11-2 (27-37 cm), S1., R42/2 (150X) 8: 146-11-2 (27-37 cm), S1., V19/4 (255X) 9: $146-11-2(27-37 \mathrm{~cm}), \mathrm{S} 1 ., \mathrm{N} 40 / 2(255 \mathrm{X})$ 10: $146-11-1(85-100 \mathrm{~cm}), \mathrm{S} 1.1, \mathrm{~L} 47 / 0(165 \mathrm{X})$ 11: $146-11-2(27-37 \mathrm{~cm}), \mathrm{S} 1 ., \mathrm{U} 21 / 1(255 \mathrm{X})$ 12: $146-11-2(27-37 \mathrm{~cm}), \mathrm{S} 1 ., \mathrm{N} 45 / 3(255 \mathrm{X})$

Figures 13-15 Unidentified Cretaceous radiolarians 13: 150-7-soup, S1.1, U44/2 (255X) 14: 150-7-soup, S1.2, 043/0 (255X) 15: 150-7-soup, S1.2, L36/2 (255X) 
PLATE 4
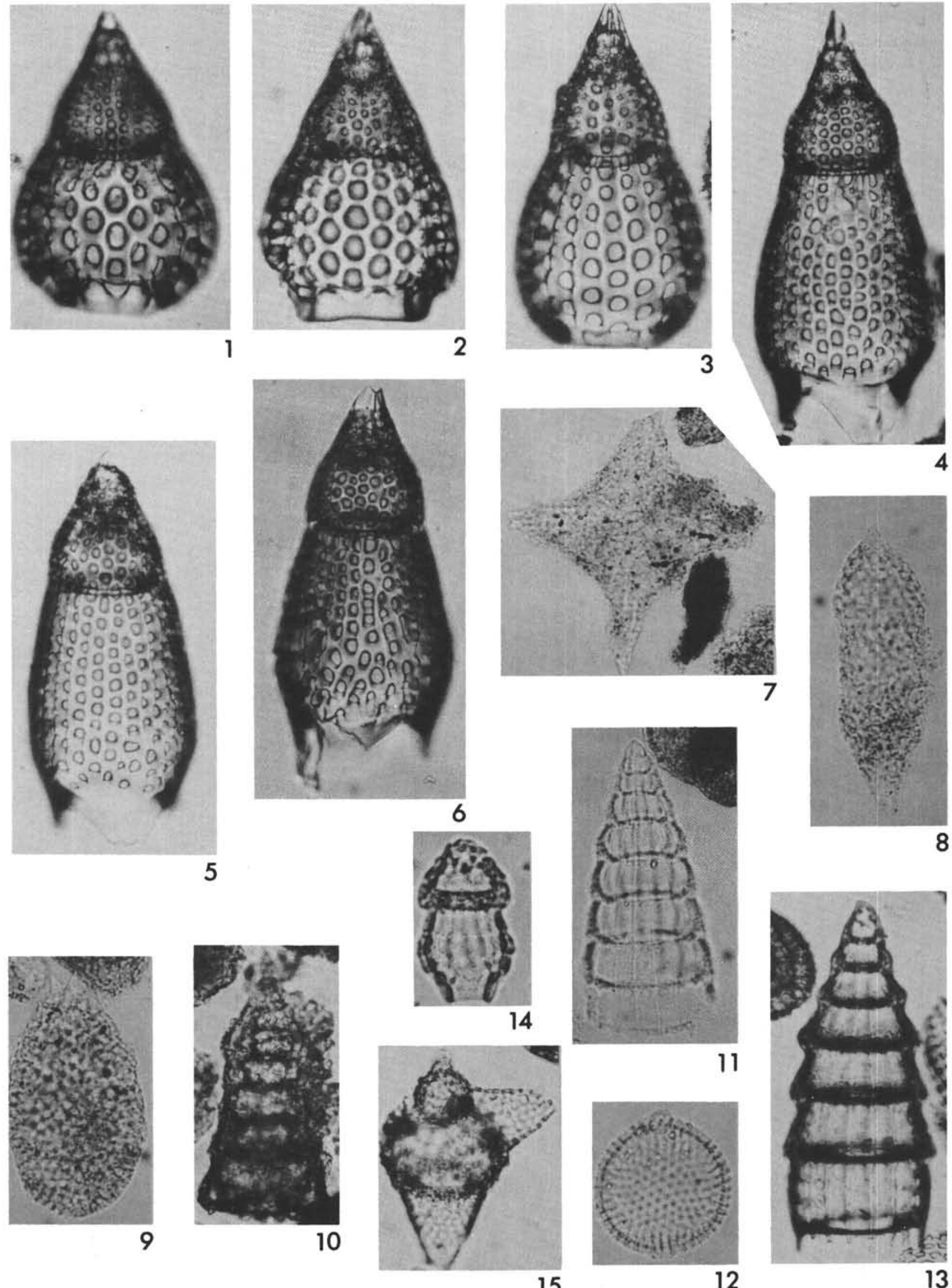
PLATE 5

Figure 1

Figures 2, 3

Figure 4

Figure 5

Figures 6, 7

Figure 10
Lithochytris archaea 150-7-soup, S1.1, P13/4 (150X)

Tepka sp. (see footnote a to Table 5)

2: $151-6-1(90-92 \mathrm{~cm}), \mathrm{S} 1.1, \mathrm{Q} 8 / 0(255 \mathrm{X})$

3: $151-6-2(77-80 \mathrm{~cm}), \mathrm{S} 1.1,046 / 1(255 \mathrm{X})$

Theocorys (?) cf. T(?) phyzella (see footnote a to Table 6). 152-4-2 (85-87 cm), S1.2, Q26/3 (255X)

Buryella tetradica with subdivided fourth segment (see footnote $b$ to Table 6) $152-7-4(145-147 \mathrm{~cm}$ ), $\mathrm{S} 1.2, \mathrm{~V} 31 / 2(255 \mathrm{X})$

Two views of the same specimen of a form believed to be ancestral to Thyrsocyrtis rhizodon (see footnote $\mathrm{c}$ to Table 6). 152-1-1 (91-93 cm), Cse.1, K9/1 (255X)

Figures 8,9 Astrosphaerin (?) spine with uniquely looped branches (see footnote $d$ to Table 6)

8: 152-3-5 (133-135 cm), Cse.1, C21/0 (150X)

9: Temporary preparation made by Peter Lonsdale, from the core-catcher sample of Core 80 of Woods Hole Oceanographic Institution's expedition CHAIN 100 collected at lat. $08^{\circ} 18.8^{\prime} \mathrm{S}$, long. $168^{\circ} 32.3^{\prime} \mathrm{W}$, in 4732 meters of water.

Lamptonium (?) incohatum (?) (see footnote e to Table 6). 152-7-3 (126-129 cm), Cse.2, W38/1 $(150 \times)$ 
PLATE 5
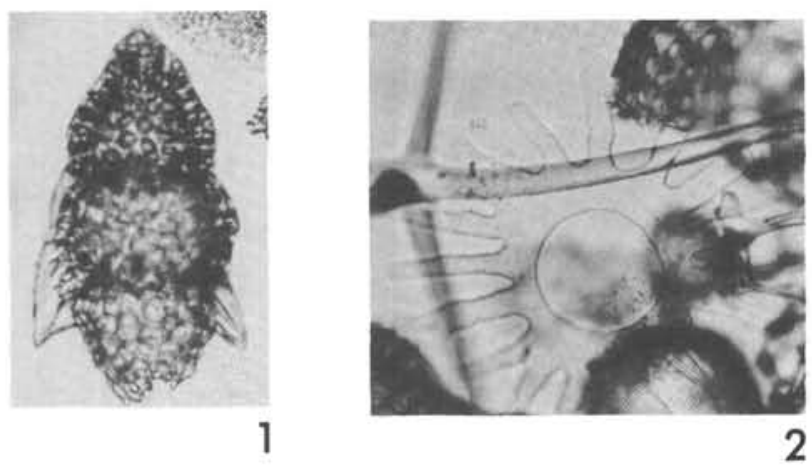

2
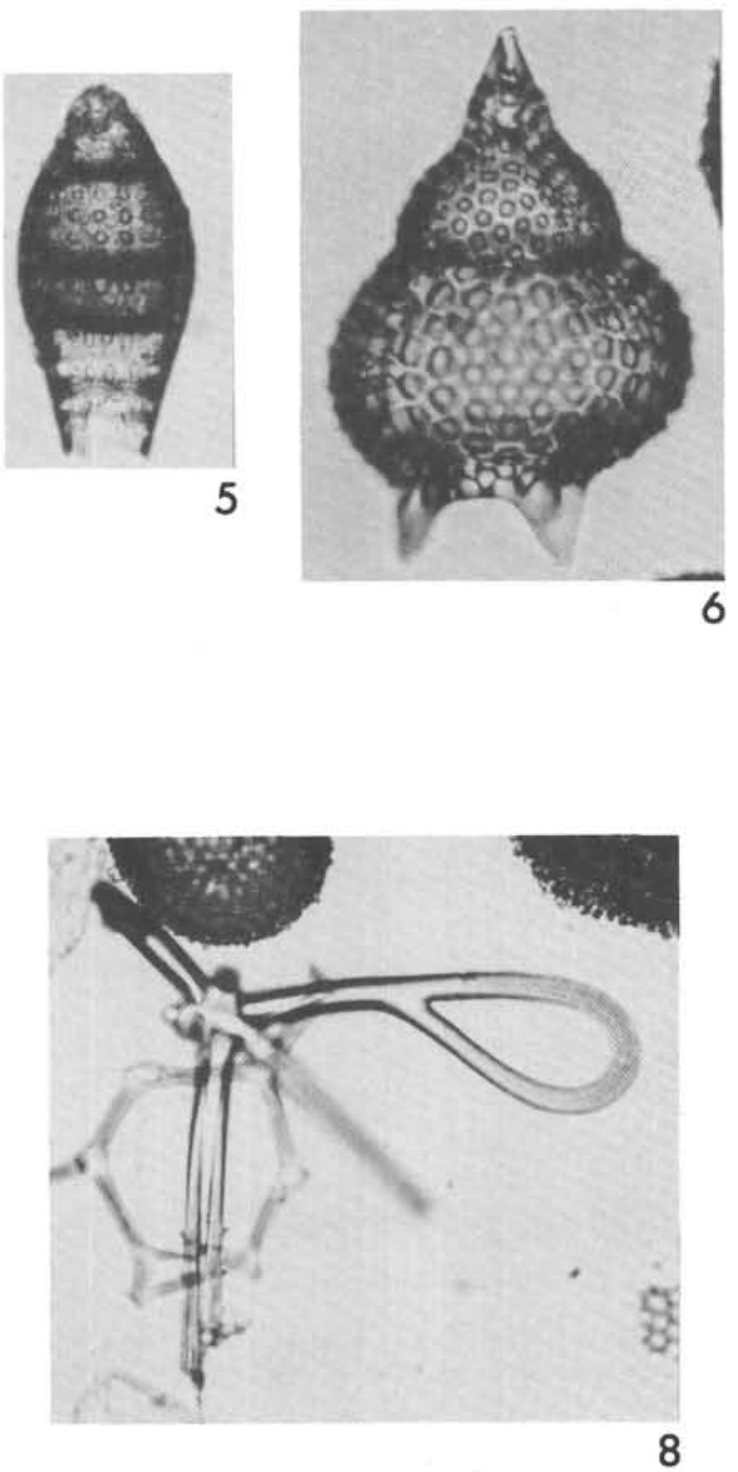
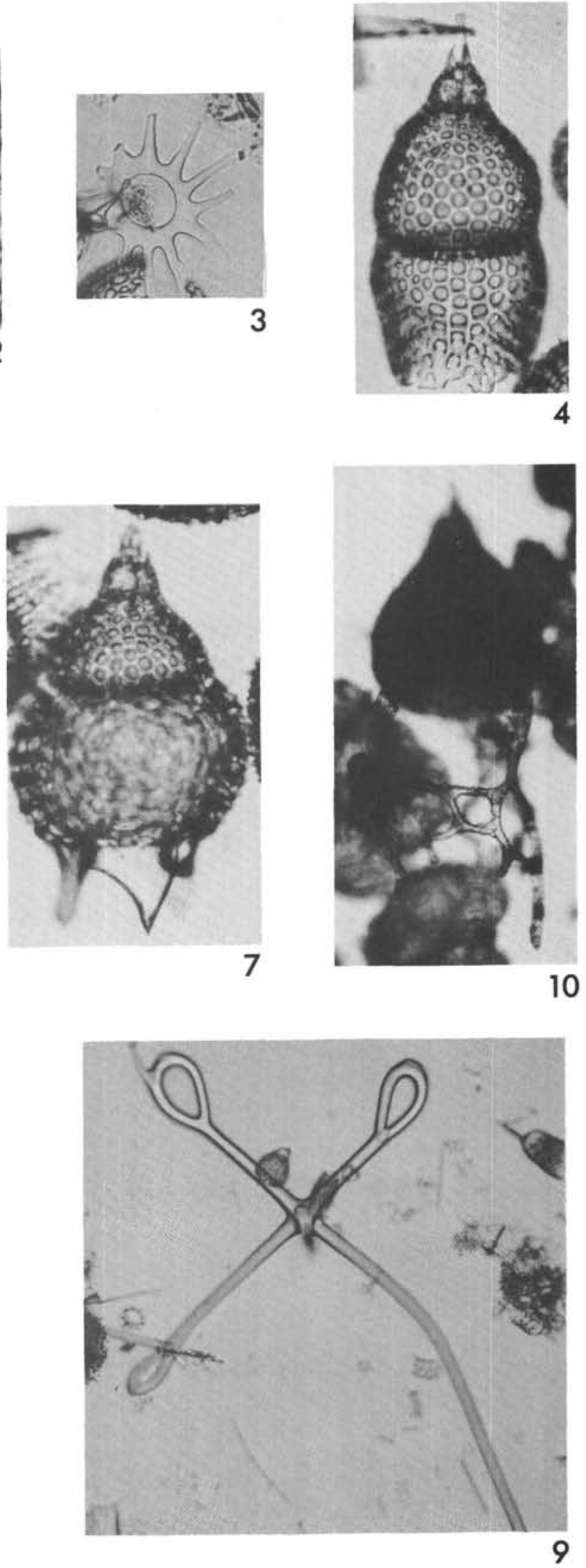